Document downloaded from:

http://hdl.handle.net/10251/45613

This paper must be cited as:

Li ., L.; Zhou ., H.; Gómez-Hernández, JJ. (2011). A Comparative Study of ThreeDimensional Hydraulic Conductivity Upscaling at the MAcro-Dispersion Experiment (MADE) site, Columbus Air Force Base, Mississippi (USA). Journal of Hydrology. 404(34):278-293. doi:10.1016/j.jhydrol.2011.05.001.

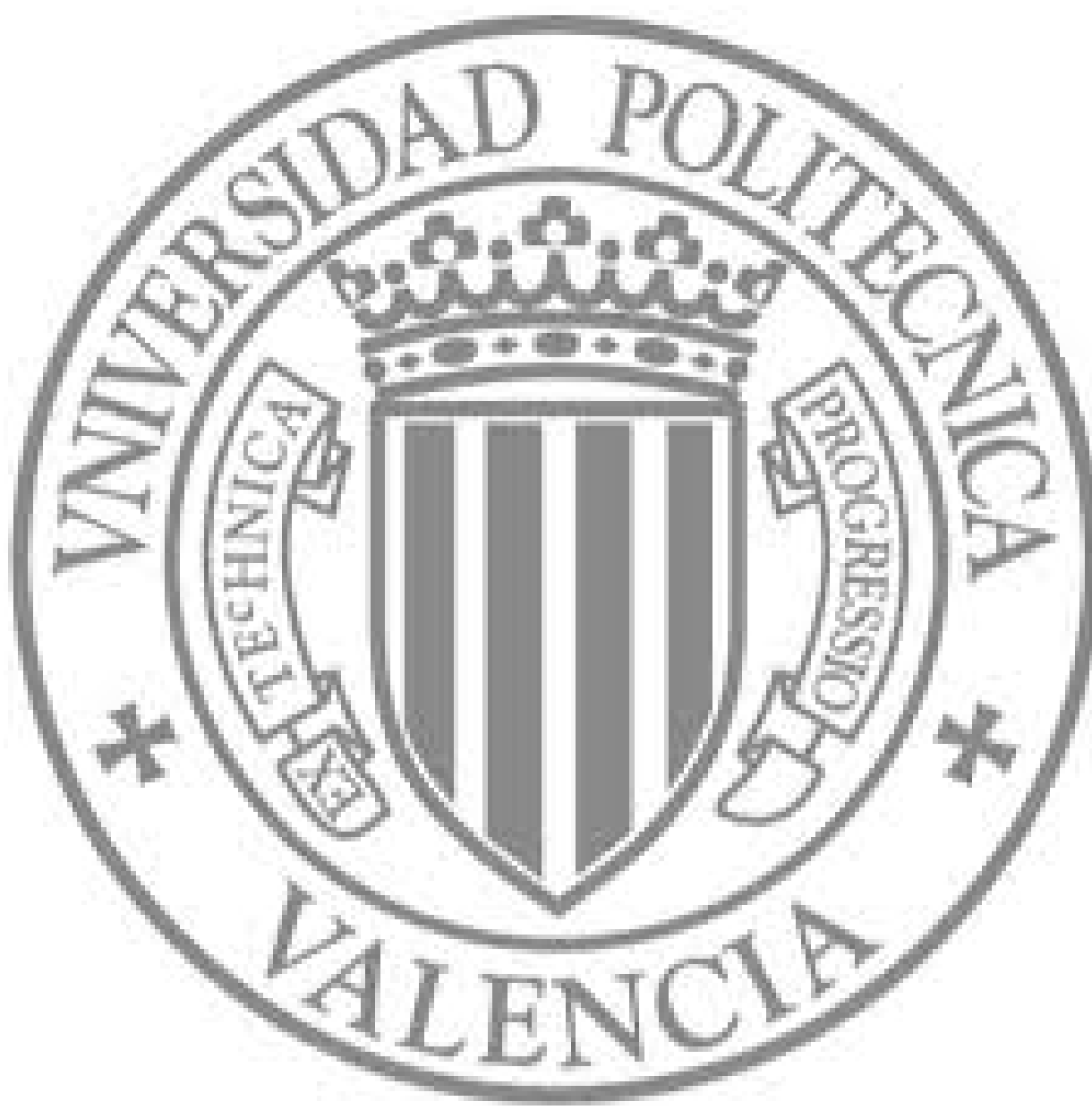

The final publication is available at

http://dx.doi.org/10.1016/j.jhydrol.2011.05.001

Copyright Elsevier 


\title{
A Comparative Study of Three-Dimensional Hydraulic Conductivity Upscaling at the MAcro-Dispersion Experiment (MADE) site, Columbus Air Force Base, Mississippi (USA)
}

\author{
Liangping Li ${ }^{a, *}$, Haiyan Zhou ${ }^{a}$, J. Jaime Gómez-Hernández ${ }^{\mathrm{a}}$ \\ ${ }^{a}$ Group of Hydrogeology, Universitat Politècnica de València, Camino de Vera, s/n, 46022 Valencia, Spain
}

\begin{abstract}
Simple averaging, simple-Laplacian, Laplacian-with-skin, and non-uniform coarsening are the techniques investigated in this comparative study of three-dimensional hydraulic conductivity upscaling. The reference is a fine scale conditional realization of the hydraulic conductivities at the MAcro-Dispersion Experiment site on Columbus Air Force Base in Mississippi (USA). This realization was generated using a hole-effect variogram model and it was shown that flow and transport modeling in this realization (at this scale) can reproduce the observed non-Fickian spreading of the tritium plume. The purpose of this work is twofold, first to compare the effectiveness of different upscaling techniques in yielding upscaled models able to reproduce the observed transport behavior, and second to demonstrate and analyze the conditions under which flow upscaling can provide a coarse model in which the standard advection-dispersion equation can be used to model transport in seemingly non-Fickian scenarios. Specifically, the use of the Laplacian-with-skin upscaling technique coupled with a non-uniform coarsening scheme yields the best results both in terms of flow and transport reproduction, for this case study in which the coarse blocks are smaller than the correlation ranges of the fine scale conductivities.

Keywords: full tensor, upscaling, interblock, non-uniform coarsening, MADE site, non-Fickian behavior
\end{abstract}

\section{Introduction}

In the last decades, two large-scale natural-gradient tracer tests were conducted to enhance the understanding of solute transport in highly heterogenous aquifers. These experiments were conducted at the Columbus Air Force Base in Mississippi, where the hydraulic conductivity variability is very high, with $\sigma_{\operatorname{lnK}}^{2} \approx 4.5$ (Rehfeldt et al., 1992). The site and the experiments performed are commonly referred to as

\footnotetext{
${ }^{*}$ Corresponding author. Tel: +34 963879615 Fax: +34 963879492

Email addresses: liali@upvnet.upv.es (Liangping Li), haizh@upvnet.upv.es (Haiyan Zhou), jaime@dihma.upv.es (J. Jaime Gómez-Hernández)
} 
MADE (MAcro-Dispersion Experiment). The present analysis focuses on the second experiment, which was performed between June 1990 and September 1991 using tritium as a non-reactive tracer. The aim of the experiment was to develop an extensive field database for validating the type of geochemical models used to predict the transport and fate of groundwater contaminants (Boggs et al., 1993). The observed tritium plume exhibits a strongly non-Fickian, highly asymmetric spreading (at the formation scale) with high concentrations maintained near the source injection area and extensive low concentrations downstream. Although there exists abundant literature on the modeling of the (so termed) anomalous spreading at the MADE site, only a few works related with this paper will be referred to in this introduction. These works can be classified into two groups according to the approach used for transport modeling.

In a first group, a number of authors have employed the classical advection-dispersion equation (ADE) to describe the strongly non-Fickian transport behavior (e.g., Adams and Gelhar, 1992; Eggleston and Rojstaczer, 1998; Barlebo et al., 2004; Salamon et al., 2007). Of these works, Salamon et al. (2007) showed that, with proper modeling of the fine-scale variability, it is possible to generate realizations of the hydraulic conductivity capable to reproduce the observed tracer movement, simply using the ADE. They used a holeeffect variogram model to characterize the flowmeter-derived conductivities. The final realizations displayed the apparent periodicity of the observed conductivities, which was enough to induce the type of spreading observed in the experiment. However, in practice, it is difficult to work with this type of high-resolution models, involving millions of nodes, particularly if multiple realizations are to be analyzed. This difficulty is what motivates our paper.

In a second group, researchers have used models that go beyond the advection-dispersion model (e.g., Berkowitz and Scher, 1998; Feehley et al., 2000; Harvey and Gorelick, 2000; Benson et al., 2001; Baeumer et al., 2001; Schumer et al., 2003; Guan et al., 2008; Liu et al., 2008; Llopis-Albert and Capilla, 2009). These authors use dual-domain mass transfer models, continuous time random walk or other alternative models capable of accounting for the strongly delayed solute transport as an alternative to the classical ADE. However, these approaches are able to provide a good match to the observed field data only a posteriori; that is, they need to calibrate their model parameters once the concentration data are collected, and then, they can reproduce, almost perfectly, any departure from Fickian transport. These works prove that there are alternative transport models able to explain the MADE data; however, at this point, they lack predictive capabilities since their parameters can only be determined after the experiment is done.

All of these studies had varying degrees of success in reproducing the spreading of the tracer plume. For instance, Barlebo et al. (2004) obtained a good reproduction of the irregular plume using the ADE after 
calibrating the concentration measurements and head data. However, calibrated hydraulic conductivities resulted a factor of five larger than the flowmeter-derived measurements. The authors attributed this discrepancy to a systematical measurement error. The accuracy of the flowmeter-derived conductivities and of the measured concentrations have raised further discussions (see Molz et al., 2006; Hill et al., 2006).

Our work builds on the study by Salamon et al. (2007) with the purpose to show that the observed transport spreading at the MADE site can also be reproduced on a coarse model by the ADE. A highresolution hydraulic conductivity realization is selected from the study by Salamon et al. (2007) and it is upscaled onto a coarser model with several orders of magnitude less elements. This upscaling approach, if successful, would permit multiple realization analyses since it would reduce significantly the computational effort needed to obtain the solute evolution at the site. Unlike previous studies of upscaling focusing on two-dimensional examples or synthetic experiments (e.g., Warren and Price, 1961; Gómez-Hernández, 1991; Durlofsky et al., 1997; Chen et al., 2003), we analyze, with real data, a variety of three-dimensional (3D) hydraulic conductivity upscaling techniques ranging from simple averaging over a uniform grid to sophisticated Laplacian-based upscaling approaches on non-uniform grids. To the best of our knowledge, this is the first time that an analysis of this type has been performed in a real 3D case. Since we will be testing the use of a full tensor representation of conductivities in the upscaled model, our group had to develop a computer code (Li et al., 2010), which has been placed on the public domain, specifically designed to solve the finite-difference approximation of the groundwater flow equation without assuming that the principal directions of the hydraulic conductivity tensors are aligned to the reference axes.

The remaining of this paper is organized as follows. First, in section 2, we summarize the findings by Salamon et al. (2007) who used a hole-effect variogram model to describe the spatial variability of $\ln K$ and, thus, were able to reproduce the non-Fickian solute spreading observed in the field. Out of the several realizations analyzed by Salamon et al. (2007), we select the one with the best reproduction of the solute spreading. This realization will be used as the reference to test different upscaling approaches. Second, in section 3, simple average, simple-Laplacian, Laplacian-with-skin and non-uniform coarsening upscaling methods are revisited from the perspective of their numerical implementation. Third, in section 4 , the flow and transport numerical models are discussed, and the benefits/limitations of using different upscaling methods at the MADE site are quantified and evaluated. Next, in section 5, there is a general discussion. Finally, in section 6, we summarize the main results and conclusions of this paper. 


\section{Modeling transport at the MADE site}

In this work, we focus on the tritium data collected in the second MADE experiment. An extensive discussion of the main geological features and hydrogeological characterization of the site has been given by Boggs et al. (1992), Adams and Gelhar (1992), Rehfeldt et al. (1992), and Boggs and Adams (1992). Salamon et al. (2007) found that the non-Fickian solute spreading observed in the field could be reproduced using the standard advection-dispersion model as long as the spatial variability of hydraulic conductivity is properly characterized at the fine scale. For the sake of completeness, next we briefly comment the results by Salamon et al. (2007).

The geostatistical analysis of the 2495 flowmeter-derived hydraulic conductivity measurements obtained at 62 boreholes (see Figure 1) indicates that the spatial variability of $\ln K$ shows a pseudo-periodic behavior in the direction of flow (Figure 2). This behavior is modeled using a hole-effect variogram, which is nested with a nugget effect and a spherical variogram as given by:

$$
\gamma(\mathbf{h})=c_{0}+c_{1} \cdot \operatorname{Sph}\left(\left\|\frac{h_{x}}{a_{x_{1}}}, \frac{h_{y}}{a_{y_{1}}}, \frac{h_{z}}{a_{z_{1}}}\right\|\right)+c_{2} \cdot\left[1-\cos \left(\left\|\frac{h_{x}}{a_{x_{2}}}, \frac{h_{y}}{a_{y_{2}}}, \frac{h_{z}}{a_{z_{2}}}\right\| \pi\right)\right]
$$

where $\mathbf{h}=\left(h_{x}, h_{y}, h_{z}\right)$ is the separation vector, $a_{x_{1}}, a_{y_{1}}, a_{z_{1}}$ are the ranges of the spherical variogram, $a_{x_{2}}, a_{y_{2}}, a_{z_{2}}$ are the ranges of the hole-effect variogram, $\|\cdot\|$ denotes vector modulus, $c_{0}$ is the nugget, $c_{1}$ is the sill of the spherical model, $c_{2}$ is the sill of the hole-effect model, with the $y$-axis oriented parallel to the flow direction, the $x$-axis is orthogonal to it on the horizontal plane, and the $z$-axis is parallel to the vertical direction. The parameter values used to fit the experimental variogram are given in Table 1. Notice that $a_{y_{2}}$, and $a_{z_{2}}$ are equal to infinity, meaning that the hole-effect is only present along the flow direction. The fitted model is also shown in Figure 2.

The computational domain is a parallelepiped with dimensions of $x=110 \mathrm{~m}, y=280 \mathrm{~m}, z=10.5 \mathrm{~m}$ and it is discretized in 2156000 cells of size $\Delta x=\Delta y=1.0 \mathrm{~m}$, and $\Delta z=0.15 \mathrm{~m}$ (see Figure 1). Cell size, according to Salamon et al. (2007), is similar in magnitude with the support scale of the flowmeter measurements. The aquifer is modeled as confined with impermeable boundaries on the faces parallel to flow, and constant head boundaries on the faces orthogonal to it. The values prescribed at the constant head boundaries are obtained by kriging the head averages over one-year observed in the nearby piezometers.

Salamon et al. (2007) used the random walk particle tracking code RW3D (Fernàndez-Garcia et al., 2005) to simulate solute transport. The local-scale longitudinal dispersivity was set as $0.1 \mathrm{~m}$, which corresponds approximately to the value calculated by Harvey and Gorelick (2000). Transverse horizontal and vertical 
local-scale dispersivity values were chosen to be one order of magnitude smaller than the longitudinal dispersivity, i.e., $0.01 \mathrm{~m}$. Apparent diffusion for tritium was set to $1.0 \mathrm{~cm}^{2} / \mathrm{d}$ (Gillham et al., 1984). An average total porosity of 0.32 as determined from the soil cores by Boggs et al. (1992) was assigned uniformly to the entire model area. The observed mass distribution on the $27^{\text {th }}$ day was employed to establish the initial concentration distribution. A simple interpolation of the initial concentrations was used to establish the concentrations in the model cells, and then 50000 particles were distributed accordingly. The observed mass distribution on the $328^{\text {th }}$ day was used to obtain reference mass profile distributions to which the model is compared. These longitudinal profiles were obtained by integrating the mass from 28 equally-spaced vertical slices, each of $10 \mathrm{~m}$ width and parallel to flow. All results are displayed after normalizing the mass by the total mass injected. Figure 3 shows the longitudinal mass distribution profiles obtained by Salamon et al. (2007) after transport simulation on 40 realizations generated by sequential Gaussian simulation. These realizations were generated using the code GCOSIM3D, (Gómez-Hernández and Journel, 1993) with the variogram model given by equation (1) and the parameter values from Table 1 . Out of these 40 realizations, solute transport on realization number 26 shows a spatial spread similar to the one observed in the field. For this reason, this conductivity realization is chosen as the reference field to test the different upscaling methods. Figure 4 shows the hydraulic conductivity field of realization number 26.

Up to here, we have limited ourselves to briefly describe the specific results from Salamon et al. (2007) that this work uses as starting point. We are not trying to re-analyze MADE, but rather to demonstrate that careful hydraulic conductivity upscaling can be used to model flow and transport in highly heterogeneous fields exhibiting, at the formation scale, a non-Fickian behavior. To evaluate the upscaling procedure we will compare flow and transport in realization \#26 before and after upscaling, aiming at obtaining the same results. Obviously, the departure of transport results computed on realization \#26 from the experimental data will remain after upscaling. Trying to get the best reproduction of the experimental data will require a further calibration exercise that is not the objective of this paper.

\section{Hydraulic conductivity upscaling}

Although hydraulic conductivity upscaling has been disregarded by some researchers on the basis that the increase of computer capabilities will make it unnecessary, there will always be a discrepancy between the scale at which we can characterize the medium, and the scale at which we can run the numerical codes. This discrepancy makes upscaling necessary to transfer the information collected at the measurement scale into a coarser scale suitable for numerical modeling. The need for upscaling is even more justified when performing 
uncertainty analysis in a Monte Carlo framework requiring the evaluation of multiple realizations. Excellent reviews on upscaling geology and hydraulic conductivity are given by Wen and Gómez-Hernández (1996b), Renard and Marsily (1997) and Sánchez-Vila et al. (2006). In this section, we briefly revisit the most commonly used upscaling techniques with an emphasis on their numerical implementation procedures.

\subsection{Simple averaging}

It is well known that, for one-dimensional flow in a heterogeneous aquifer, the equivalent hydraulic conductivity $\left(K^{b}\right)$ that, for a given hydraulic head gradient, preserves the flows crossing the aquifer is given by the harmonic mean of the hydraulic conductivities (Freeze and Cherry, 1979). In two-dimensional flow for media with isotropic spatial correlation and a lognormal probability distribution, the geometric mean provides good block conductivities (Matheron, 1967); Gómez-Hernández and Wen (1994) and Sánchez-Vila et al. (1996) used synthetic experiments to corroborate this conclusion.

Some heuristic rules have been proposed for three-dimensional upscaling. Cardwell and Parsons (1945) had already shown that the block conductivity should lie between the arithmetic mean and the harmonic mean when Journel et al. (1986) proposed the use of power averages (also referred to as $\omega$-norms) to estimate block conductivities. The power average is given by:

$$
K^{b}=\left\{\frac{1}{V(\mathbf{x})} \int_{V(\mathbf{x})}\left(K_{x}\right)^{\omega} d V\right\}^{1 / \omega}
$$

where $V(\mathbf{x})$ indicates the volume of the block; $K^{b}$ is the block conductivity, and $K_{x}$ represents the cell conductivities within the block, the power $\omega$ may vary from -1 , yielding the harmonic mean, to +1 , yielding the arithmetic mean, with $\omega=0$ corresponding to the geometric mean. Although Desbarats (1992) demonstrated that $\omega$ equals $1 / 3$ in $3 \mathrm{D}$ for statistically isotropic and mildly heterogeneous formations, the power coefficient $(\omega)$ has to be obtained by resorting to numerical flow experiments in arbitrary flow fields. The main advantages of this method are its mathematic conciseness and the easiness of implementation. However, there are several limitations to this power-average approach: first, the exponent $\omega$ is site-specific and cannot be predicted in a general anisotropic heterogeneous medium except after numerical calibration experiments; second, the shape and size of the blocks are not considered.

\subsection{Simple-Laplacian}

This approach is based on the local solution, for each block being upscaled, of a variant of the Laplace equation (steady-state, groundwater flow with neither sources nor sinks). In this approach, the block con- 
ductivity is assumed to be a tensor with principal directions parallel to the coordinate axes; and therefore, diagonal for this reference system.

To determine each component of the tensor, a local problem is solved inducing flow in the component direction. For instance, in $2 \mathrm{D}$, the tensor will have two components, $K_{x x}^{b}$, and $K_{y y}^{b}$; to determine the component corresponding to the $x$ direction, $K_{x x}^{b}$, the procedure would be as follows: (1) extract the block being upscaled and solve the groundwater flow equation just within the block, at the fine scale with no flow boundaries on the sides parallel to flow and prescribed heads on the sides perpendicular to flow as shown in Figure 5; (2) evaluate the total flow $Q$ through any cross-section parallel to the $y$-axis from the solution of the flow equation, and (3) compute the block conductivity tensor component in the $x$-direction as:

$$
K_{x x}^{b}=-\left(\frac{Q}{y_{1}-y_{0}}\right) /\left(\frac{h_{1}-h_{0}}{x_{1}-x_{0}}\right)
$$

where $y_{1}-y_{0}$ is the block width; $h_{1}-h_{0}$ is the difference between the prescribed heads on the opposite sides of the block (see Figure 5), and $x_{1}-x_{0}$ is the block length. $K_{y y}^{b}$ would be obtained similarly after solving a similar local flow problem with the boundary conditions in Figure 5 rotated $90^{\circ}$.

The main shortcoming of this approach is that the assumption of a diagonal tensor is not well-founded for a heterogeneous aquifer. In other words, the heterogeneity within the block may induce an overall flux that is not parallel to the macroscopic head gradient, a behavior that cannot be captured with a diagonal tensor

This method has been widely used to calculate block conductivities in petroleum engineering and hydrogeology (e.g., Warren and Price, 1961; Bouwer, 1969; Journel et al., 1986; Desbarats, 1987, 1988; Deutsch, 1989; Begg et al., 1989; Bachu and Cuthiell, 1990). More recently Sánchez-Vila et al. (1996) utilized this approach to study the scale effects in transmissivity; Jourde et al. (2002) used it to calculate block equivalent conductivities for fault zones; and Flodin et al. (2004) used this method to illustrate the impact of boundary conditions on upscaling. It has also been employed by Fernàndez-Garcia and Gómez-Hernández (2007) and Fernàndez-Garcia et al. (2009) to evaluate the impact of hydraulic conductivity upscaling on solute transport. Some reasons favoring this approach are that it is not empirical but phenomenological, i.e., it is based on the solution of the groundwater flow equation, and it yields a tensor representation of the block conductivity, which would be exact for the case of perfectly layered media, with the layers parallel to the coordinate axes. 


\subsection{Laplacian-with-skin}

To overcome the shortcomings of the simple-Laplacian approach, the Laplacian-with-skin approach was presented by Gómez-Hernández (1991). In this approach, the block conductivity is represented by a generic tensor (not necessarily diagonal) and the local flow problem is solved over an area that includes the block plus a skin surrounding it (see Figure 6). The skin is designed to reduce the impact of the arbitrary boundary conditions used in the solution of the local flow problems letting the conductivity values surrounding the block to take some control on the flow patterns within the block.

For a 3D block, the overall algorithm is summarized as follows: (1) the block to upscale plus the skin is extracted from the domain; (2) flow is solved at the fine scale within the block-plus-skin region for a series of boundary conditions; (3) for each boundary condition the spatially-averaged specific discharge (q) and gradient $(\mathbf{J})$ are calculated as,

$$
\left\langle q_{i}\right\rangle=\frac{1}{V(\mathbf{x})} \int_{V(\mathbf{x})} q_{i}(\mathbf{x}) d \mathbf{x}
$$

$$
\left\langle J_{i}\right\rangle=\frac{1}{V(\mathbf{x})} \int_{V(\mathbf{x})} \frac{\partial h(\mathbf{x})}{\partial x_{i}} d \mathbf{x}
$$

where $i$ refers to the three components of the vectors (i.e., $q_{x}, q_{y}$ and $q_{z} ; J_{x}, J_{y}$ and $J_{z}$ ); and (4) the tensor components of $\mathbf{K}^{b}$ are determined by solving the following overdetermined system of linear equations by a standard least squares procedure (Press et al., 1988).

$$
\left[\begin{array}{cccccc}
\left\langle J_{x}\right\rangle_{1} & \left\langle J_{y}\right\rangle_{1} & \left\langle J_{z}\right\rangle_{1} & 0 & 0 & 0 \\
0 & \left\langle J_{x}\right\rangle_{1} & 0 & \left\langle J_{y}\right\rangle_{1} & \left\langle J_{z}\right\rangle_{1} & 0 \\
0 & 0 & \left\langle J_{x}\right\rangle_{1} & 0 & \left\langle J_{y}\right\rangle_{1} & \left\langle J_{z}\right\rangle_{1} \\
\left\langle J_{x}\right\rangle_{2} & \left\langle J_{y}\right\rangle_{2} & \left\langle J_{z}\right\rangle_{2} & 0 & 0 & 0 \\
0 & \left\langle J_{x}\right\rangle_{2} & 0 & \left\langle J_{y}\right\rangle_{2} & \left\langle J_{z}\right\rangle_{2} & 0 \\
0 & 0 & \left\langle J_{x}\right\rangle_{2} & 0 & \left\langle J_{y}\right\rangle_{2} & \left\langle J_{z}\right\rangle_{2} \\
\cdots & \cdots & \ldots & \cdots & \cdots & \cdots \\
\left\langle J_{x}\right\rangle_{n} & \left\langle J_{y}\right\rangle_{n} & \left\langle J_{z}\right\rangle_{n} & 0 & 0 & 0 \\
0 & \left\langle J_{x}\right\rangle_{n} & 0 & \left\langle J_{y}\right\rangle_{n} & \left\langle J_{z}\right\rangle_{n} & 0 \\
0 & 0 & \left\langle J_{x}\right\rangle_{n} & 0 & \left\langle J_{y}\right\rangle_{n} & \left\langle J_{z}\right\rangle_{n}
\end{array}\right] \cdot\left[\begin{array}{c}
K_{x x}^{b} \\
K_{x y}^{b} \\
K_{x z}^{b} \\
K_{y y}^{b} \\
K_{y z}^{b} \\
K_{z z}^{b}
\end{array}\right]=-\left[\begin{array}{c}
\left\langle q_{x}\right\rangle_{1} \\
\left\langle q_{y}\right\rangle_{1} \\
\left\langle q_{z}\right\rangle_{1} \\
\left\langle q_{x}\right\rangle_{2} \\
\left\langle q_{y}\right\rangle_{2} \\
\left\langle q_{z}\right\rangle_{2} \\
\cdots \\
\left\langle q_{x}\right\rangle_{n} \\
\left\langle q_{y}\right\rangle_{n} \\
\left\langle q_{z}\right\rangle_{n}
\end{array}\right]
$$

where $1, \ldots, n$ refers to the different boundary conditions; $K_{x x}^{b} \cdots K_{z z}^{b}$ are the components of the upscaled equivalent conductivity tensor $\mathbf{K}^{b}$. In principle, in $3 \mathrm{D}$, two sets of boundary conditions are sufficient to 
determine $\mathbf{K}^{b}$. However, from a practical point of view, the number of boundary conditions should be greater than two $(n>2)$ to better approximate all possible flow scenarios.

Every three rows in Equation (6) are the result of enforcing Darcy's law on the average values in equations (4) and (5) for a given boundary condition:

$$
\langle\mathbf{q}\rangle=-\mathbf{K}^{b}\langle\mathbf{J}\rangle
$$

The block conductivity tensor must be symmetric and positive definite. Symmetry is easily enforced by making $K_{x y}^{b}=K_{y x}^{b}, K_{x z}^{b}=K_{z x}^{b}$ and $K_{y z}^{b}=K_{z y}^{b}$. Positive definiteness is checked a posteriori. In case the resulting tensor is non-positive definite, the calculation is repeated either with more boundary conditions or with a larger skin size (Wen et al., 2003; Li et al., 2011).

We note that the critical point in this approach is the selection of the set of $n$ alternative boundary conditions. In general, this set of boundary conditions is chosen so as to induce flow in several directions (for instance, the prescribed head boundary conditions in Figure 6 induce flow at $0^{\circ}, 45^{\circ}, 90^{\circ}$ and $135^{\circ}$ angles with respect to the $x$-direction). For the boundary conditions, we have chosen to prescribe linearly varying heads along the sides of the blocks, other authors (Durlofsky, 1991) have proposed the use of periodic boundary conditions. Flodin et al. (2004) showed that the resulting block conductivities do not depend significantly on whether the boundary conditions are linearly varying or periodic.

\subsection{Non-uniform coarsening}

Prior to upscaling, the fine-scale realization has to be overlain with the coarse-scale discretization that will be used in the numerical model. Each block in the coarse discretization must be assigned an upscaled conductivity value on the basis of the conductivity values in the fine-scale realization. Initially, all studies on hydraulic conductivity upscaling assumed that the coarse scale discretization was uniform, that is, all coarse blocks were of the same shape and size, until Durlofsky et al. (1997) introduced the concept of nonuniform coarsening. The rationale was simple, if upscaling induces smoothing, and the petroleum engineer is most interested in the water cut (the early breakthrough at the production wells when petroleum is being displaced by injected water) it is important to smooth the least the areas of high displacement velocities, whereas the smoothing in the areas of low velocities is less relevant. For this purpose, Durlofsky et al. (1997) suggest the following steps: (1) identify the underlying high velocity regions using a fine-scale single-phase flow simulation; (2) on the basis of this simulation define a discretization with small blocks in high-velocity areas and large ones elsewhere; and (3) apply the Laplacian-with-skin upscaling technique to calculate the 
block conductivity tensors of the coarse (non-uniform) blocks.

In a hydrogeological context, we can also use a non-uniform coarsening aimed to preserve small blocks in: (1) high flow velocity zones; (2) regions where hydraulic gradients change substantially over short distances, such as near pumping or injection wells (Wen and Gómez-Hernández, 1998); (3) areas near contaminant spills within a regional aquifer where accurate simulation of plume movement is of interest; and (4) in zones requiring a detailed representation of heterogeneity, for instance to capture channels or fractures (Durlofsky et al., 1997; Wen et al., 2003; Flodin et al., 2004).

\section{Coarse model and simulation results}

In this section, we first present the governing equation and the solution procedures for the flow and transport models, and then we discuss the results obtained applying the different upscaling techniques described in the previous section. All of these techniques are applied to realization \#26 of the MADE aquifer in Salamon et al. (2007).

\subsection{Coarse Flow and Transport Equations}

Under steady-state flow conditions and in the absence of sinks and sources, the flow equation of an incompressible or slightly compressible fluid in saturated porous media can be expressed by combining Darcy's Law and the continuity equation, which in Cartesian coordinates is (Bear, 1972; Freeze and Cherry, 1979):

$$
\nabla \cdot(\mathbf{K}(\mathbf{x}) \nabla h(\mathbf{x}))=0
$$

where $h$ is the piezometric head, and $\mathbf{K}$ is a second-order symmetric hydraulic conductivity tensor.

Most frequently, the hydraulic conductivity tensor is assumed isotropic and therefore can be represented by a scalar. In this case, a standard seven-point block-centered finite-difference stencil is typically employed to solve the partial differential equation in three dimensions. This approach is also valid if, for all blocks, the conductivity is modeled as a tensor with the principal directions aligned with the block sides (Harbaugh et al., 2000). However, when modeling geologically complex environments at a coarse scale, the assumption of isotropic block conductivity or even tensor conductivity with principal components parallel to the block sides is not warranted. It is more appropriate to use a full hydraulic conductivity tensor to capture properly the average flow patterns within the blocks (Bourgeat, 1984; Gómez-Hernández, 1991; Wen et al., 2003; Zhou et al., 2010). Recently, the commonly used groundwater model software MODFLOW implemented a new 
module that allows the use of a full tensorial representation for hydraulic conductivity within model layers (Anderman et al., 2002) which has been successfully applied in 2D examples such as in Fernàndez-Garcia and Gómez-Hernández (2007).

Modeling three-dimensional flow in a highly heterogeneous environment at a coarse scale, requires accounting for a tensorial representation of hydraulic conductivity. We cannot assume, a priori that specific discharge and hydraulic head gradient will be parallel, nor that the principal directions of the hydraulic conductivity tensors are the same in all blocks. For this reason, and given that MODFLOW can only account for 3D tensors if one of its principal directions is aligned with the vertical direction, Li et al. (2010) developed a three-dimensional groundwater flow simulation with tensor conductivities of arbitrary orientation of their principal directions. This code is based on an nineteen-point finite-difference approximation of the groundwater flow equation, so that the flow crossing any block interface will depend not only on the head gradient orthogonal to the face, but also on the head gradient parallel to it.

Finite-difference modeling approximates the specific discharges across the interface between any two blocks $i$ and $j$ as a function of the hydraulic conductivity tensor in between block centers. This tensor is neither the one of block $i$ nor of the one of block $j$. For this reason, finite-difference numerical models need to approximate the interblock conductivity; the most commonly used approximation is taking the harmonic mean of adjacent block values. When block conductivities are represented by a tensor, the concept of how to average the block tensors in adjacent blocks is not clear. To overcome this difficulty, the code developed by Li et al. (2010) takes directly, as input, interblock conductivity tensors, removing the need of any internal averaging of tensors defined at block centers. Within the context of upscaling, deriving the interblock conductivity tensors simply amounts to isolate the parallelepiped centered at the interface between adjacent blocks, instead of isolating the block itself, and then apply the upscaling techniques described in the previous section. In other contexts, the user must supply the interblock conductivity tensors directly. Several authors (Appel, 1976; Gómez-Hernández, 1991; Romeu and Noetinger, 1995; Li et al., 2010) have recommended to work directly with interblock conductivities for more accurate groundwater flow simulations.

The details of the algorithm used to solve the flow equation are provided in Li et al. (2010) and summarized in Appendix A.

Mass transport is simulated using the advection-dispersion equation: (Bear, 1972; Freeze and Cherry, 1979):

$$
\phi \frac{\partial C(\mathbf{x}, t)}{\partial t}=-\nabla \cdot(\mathbf{q}(\mathbf{x}) C(\mathbf{x}, t))+\nabla \cdot(\phi \mathbf{D} \nabla C(\mathbf{x}, t))
$$


where $C$ is the dissolved concentration of solute in the liquid phase; $\phi$ is the porosity; $\mathbf{D}$ is the local hydrodynamic dispersion coefficient tensor, and $\mathbf{q}$ is the Darcy velocity given by $\mathbf{q}(\mathbf{x})=-\mathbf{K}(\mathbf{x}) \nabla h(x)$.

As in the works of Salamon et al. (2007) and Llopis-Albert and Capilla (2009) at the MADE site, the random walk particle tracking code RW3D (Fernàndez-Garcia et al., 2005; Salamon et al., 2006) is used to solve the transport equation (9). In this approach, the displacement of each particle in a time step includes a deterministic component, which depends only on the local velocity field, and a Brownian motion component responsible for dispersion. A hybrid scheme is utilized for the velocity interpolation which provides local as well as global divergence-free velocity fields within the solution domain. Meanwhile, a continuous dispersion-tensor field provides a good mass balance at grid interfaces of adjacent cells with contrasting hydraulic conductivities (LaBolle et al., 1996; Salamon et al., 2006). Furthermore, in contrast to the constant time scheme, a constant displacement scheme (Wen and Gómez-Hernández, 1996a), which modifies automatically the time step size for each particle according to the local velocity, is employed in order to reduce computational effort.

\subsection{Upscaling design and error measure}

In this work, we have performed both uniform and non-uniform upscaling. In the case of uniform upscaling, the original hydraulic conductivity realization discretized into $110 \times 280 \times 70$ cells of $1 \mathrm{~m}$ by $1 \mathrm{~m}$ by $0.15 \mathrm{~m}$ is upscaled onto a model with $11 \times 28 \times 14$ blocks of $10 \mathrm{~m}$ by $10 \mathrm{~m}$ by $0.75 \mathrm{~m}$. This upscaling represents going from 2156000 cells down to 4312 blocks, i.e., a reduction by a factor of 500 . The reduction in model size, undoubtedly, reduces the computational cost for flow and transport modeling. As will be shown, the flow and transport results can be improved using a non-uniform discretization of the coarse model. For the non-uniform upscaling, the discretization continues to be a rectangular grid, with the following coarse block dimensions: along the $x$-axis (orthogonal to flow), block dimension is $10 \mathrm{~m}$, except between $x=40 \mathrm{~m}$ and $x=90 \mathrm{~m}$ where it is $5 \mathrm{~m}$; along the $y$-axis (parallel to flow), block dimension is 10 $\mathrm{m}$, except between $y=20 \mathrm{~m}$ and $y=130 \mathrm{~m}$ where it is $5 \mathrm{~m}$; and along the $z$-axis, block dimension is $1.5 \mathrm{~m}$ between $z=0 \mathrm{~m}$ and $z=3 \mathrm{~m}$ and $0.75 \mathrm{~m}$ elsewhere. The final model has $16 \times 39 \times 12(7488)$ blocks, with smaller blocks close to the source and along the area through which it is most likely that the solute plume will travel. The reduction factor in size, with respect to the initial discretization is close to 300 .

The first set of upscaling runs use simple averaging rules to obtain the block conductivity values. The second set of runs use the Laplacian-based approaches. Within this second set of runs we carry out a first comparison using tensor conductivity values computed at block centers versus tensor conductivities computed at the interfaces; the former requires a further averaging of adjacent block values to approximate 
the interblock conductivities needed by the numerical solver, whereas the latter does not. Then, after showing that interface-centered conductivity upscaling is more appropriate, the following upscaling runs are always performed with interblock conductivities.

In the application of any of the Laplacian approaches for upscaling, the local flow model that must be run for each block was solved by finite differences using the preconditioned conjugate gradient method implemented in MODFLOW (Hill, 1990) since we found it to be the fastest algorithm for the same convergence criteria.

In the Laplacian-with-skin approach, the size of the skin was taken equal to half the block size in each direction. A prior sensitivity analysis revealed that this skin size was enough to capture accurately the average flow crossing each of the upscaled blocks. Zhou et al. (2010) also found that half the block size is a good choice for the skin size in most situations. The overdetermined system of equations from which the components of the block tensor are described is built after solving nine local flow problems. In each of the local problems the prescribed heads applied to the boundaries of the block vary linearly as a function of $x, y$ and $z$ so that they impose overall head gradients parallel to the directions given by the following nine vectors $(1,0,0),(0,1,0),(0,0,1),(1,1,0),(1,0,1),(0,1,1),(1,1,0),(-1,0,1),(0,-1,1)$.

To evaluate the performance of the different upscaling techniques we focus on the reproduction of the interblock fluxes and on the reproduction of the solute transport. For the fluxes, we compare the interblock specific discharges obtained after solving the flow equation at the coarse scale with the corresponding values derived after solving the flow equation in the reference field at the fine scale. We focus on fluxes instead of piezometric heads because fluxes have a larger spatial variability and have a dominant role in solute transport. The metric we use to evaluate each technique is the average relative bias $(R B)$ given by:

$$
R B=\frac{1}{N} \sum_{i=1}^{N}\left|\frac{q_{f, i}-q_{c, i}}{q_{f, i}}\right| \cdot 100
$$

where $N$ is the number of block interfaces; $q_{f, i}$ is the specific discharge through the block interface $i$ computed from the fine scale solution, and $q_{c, i}$ is the specific discharge through the block interface $i$ resulting from the coarse scale simulation.

Mass transport reproduction is evaluated qualitatively by comparing the longitudinal mass distribution profiles at the $328^{\text {th }}$ day obtained from the fine scale model with the one obtained from the coarse scale model.

Notice that the same transport parameters used for the fine scale simulation described in section 2 are also used for the coarse scale simulation. 


\subsection{Results and Comparisons}

Next, we will discuss the flow and transport performance of the different upscaling approaches. The flow upscaling analysis excludes the interfaces of the blocks which are adjacent to the boundaries; the reason for the exclusion is that the boundary conditions have an impact on the results of upscaling in the nearby blocks (Vermeulen et al., 2006). Excluding these blocks, the discrepancies in flow reproduction between the coarse and fine scale simulations will be due to the upscaling method and not to the presence of the boundaries. This consideration is not necessary when analyzing the transport upscaling since the plume travels far enough from the boundaries. Also, since, for transport purposes, the flows along the $y$-axis are the most relevant (and of the highest magnitude), the graphs only shows the specific discharges across the interfaces orthogonal to the $y$-axis, similar results are obtained when analyzing the interfaces orthogonal to the $x$ - and $z$-axis.

Figure 7 shows the scatterplots of reference versus upscaled fluxes through the block interfaces using simple averaging methods. All circles within the dotted lines have a relative bias smaller than $10 \%$ of the reference values, whereas the circles within the solid lines have a relative bias smaller than $40 \%$. It is clear that, out of the different averages, the power average with a power of 0.5 gives the best results. The use of the harmonic mean (Figure 7A) (power average with $\omega=-1$ ) tends to severely underestimate the reference fluxes, while the arithmetic mean (Figure 7C) (power average with $\omega=+1$ ) tends to overestimate them. The geometric mean (power average with $\omega=0$ ) does a better work but stills tends to underestimate the fluxes (Figure 7B). The best average, as already pointed out by Cardwell and Parsons (1945) should be somewhere between the harmonic and the arithmetic averages. In this specific case, we found that the smallest bias occurs when $\omega=0.5$ (Figure 7D), resulting in a relative bias, RB, of 11\%. As mentioned earlier, for isotropic, mildly heterogeneous media, Desbarats (1992) found $\omega=1 / 3$ to be the best power average for upscaling purposes. In the MADE case, the field is neither isotropic, nor mildly varying ( $\ln K$ variance is close to 5 ), thus it is not surprising that the optimal power value does not coincide with the value reported by Desbarats (1992).

Figure 8 shows the longitudinal mass distribution profile (integrated along the direction orthogonal to flow, and normalized by the total mass) of the tritium plume using different simple averaging upscaling techniques at 328 days. The solid line represents the fine scale result. For reference, the initial conditions at 27 days are also shown by the bold dashed curve. The remaining of the curves are the upscaled results for the different averages. Both the upscaled models using the arithmetic mean and the 0.5 power average are capable of reproducing the long downstream spreading of the contaminant plume, with the power mean 
resulting in a better representation of the distribution close to the source. Yet, none of the methods exhibits a satisfactory accuracy.

Figure 9 shows the scatterplots of reference versus upscaled fluxes using different Laplacian approaches. Figures 9A and 9B display upscaling approaches using a simple-Laplacian (i.e., without skin, and assuming diagonal tensors) for block-centered and interblock-centered upscaling, respectively. It is clear that it is better to upscale directly the interblock conductivity than upscaling the block values and then let the numerical model estimate internally the interblock conductivity. This is consistent with earlier studies (Li et al., 2010).

Figures 9B and 9C display two different Laplacian approaches without skin. The simple-Laplacian in Figure 9B assumes a diagonal representation of the tensor in the reference axes, whereas the Laplacian-withskin but with a skin set to zero in Figure 9C allows for the tensor representation to be non-diagonal. Allowing the tensor principal components not to be aligned with the reference axes results in a better representation of the fluxes, since it is unlikely that all interblocks would have conductivities with principal directions parallel to the reference axes.

Moreover, if the skin is allowed to increase up to half the block size, the results improve even further, as can be checked by comparing Figures 9C and 9D. This improvement can be related to the reduction of the influence in the flow patterns within the block of the boundary conditions used in the local flow models in favor of the influence of the nearby conductivities from the reference aquifer.

Since most of the commonly available groundwater flow simulators only accept diagonal tensors as input parameter values, a test was made by solving the flow and transport in the coarse scale ignoring the offdiagonal components of the tensors used in Figure 9D. The results are shown in Figure 9E and they are qualitatively similar to those in Figure 9D. In this specific case, in which the reference axes of the numerical model are aligned with the main directions of the statistical anisotropy of hydraulic conductivity it could be expected that the off-diagonal components of the upscaled block conductivity tensors were small, and therefore, flow predictions neglecting them go almost unaffected. In a general setting with complex geology, cross-beddings, or non-uniform anisotropies, the use of a full tensor block conductivity would be necessary for a good reproduction of the aquifer response (Bierkens and Weerts, 1994).

Finally, Figure 9F shows that the best results are achieved when the upscaling is performed on a nonuniform coarse grid, which has been refined in the areas of highest velocities (see grid in Figure 15), using an interface-centered Laplacian-with-skin upscaling. While this result is expected, since the number of model blocks is larger in the non-uniform grid, the improvement is not due just to having almost twice as many blocks, but to the fact, that these many more blocks are located in the zones where the variability of velocity 
is the highest. The message to take away is that it is advantageous to use a non-uniform coarse grid and that the definition of this grid is very important to achieve the best upscaling results. Other authors have investigated along these lines and have proposed the use of flexible grids which maintain a given topology (basically keeping constant the number of rows, columns and layers) but which are deformed so as to reduce the variability of the specific discharge vector within each coarse block (i.e, Garcia et al., 1992; Wen and Gómez-Hernández, 1998).

Figure 10 compares the mass longitudinal profile of the upscaling approaches in Figures 9A (uniform grid, simple-Laplacian, block-centered), 9B (uniform grid, simple-Laplacian, interblock-centered) and 9D (uniform grid, Laplacian-with-skin, interblock-centered) with the reference profile at day 328 . The improvement in the reproduction of the reference values by the difference upscaling techniques shows a similar progression as the improvement seen in the reproduction of the fluxes in Figure 9. Comparing these curves to any of the curves in Figure 8, which were obtained with simple averaging upscaling rules, it is clear that any upscaling approach based on a local solution of the flow equation provides a better representation of the hydraulic conductivity distribution and yields better transport predictions. The two interblock-aimed upscaling approaches are able to capture both the peak concentration near the source and the downstream spreading.

Figure 11 shows the mass longitudinal profile of the upscaling approaches in Figures 9D (uniform grid, Laplacian-with-skin, interblock-centered) and 9F (non-uniform grid, Laplacian-with-skin, interblockcentered). It is evident that the non-uniform coarsening gives again the best results: up to a downstream distance of $200 \mathrm{~m}$, the reproduction is almost perfect, and the very low concentrations for distances farther than $200 \mathrm{~m}$ are adequately reproduced.

A final comparison of the different approaches can be performed by analyzing the spatial distribution of the contaminant plume, both in plan view (depth integrated) and lateral view (integrated along the $x$ axis). Figure 12 shows the contaminant plume in the reference fine-scale conductivity realization. Figures 13, 14, and 15 show the corresponding distributions for the mass transport simulation in the upscaled fields using a block-centered, simple-Laplacian upscaling approach, an interblock-centered, Laplacian-with-skin approach, and the non-uniform coarsening, interblock-centered, Laplacian-with-skin approach, respectively. It is evident that the block-centered approach is not capable to produce a field in which the solute travels as far downstream as in the reference field, while the most elaborated upscaling approach of Figure 15 gives results which quite closely resemble the reference values. 


\section{Discussion}

We have shown that flow and transport can be modeled at the MADE site by the advection dispersion equation on relatively coarse discretization if the spatial variability of hydraulic conductivity at the fine scale is properly characterized and a careful upscaling approach is applied to it. But, why is this so? and why is the non-uniform grid interblock-centered Laplacian-with-skin upscaling the approach to use?

Let's first analyze the progression in the reproduction of the specific discharges with the upscaling approaches. It is well known that the coarse-scale representation of conductivity as a tensor is mostly due to the statistical anisotropy at the fine scale (Lake, 1988). In the limit, with infinite correlation in the horizontal plane, the medium would be perfectly layered and the tensor conductivity will have arithmetic average for the horizontal components and the harmonic average for the vertical ones. At the MADE site, the horizontal continuity is not infinity, but it is quite large compared with the size of the domain, this is the reason why, for the reproduction of the specific discharges across the interfaces which are orthogonal to the direction of maximum continuity, the best average is a power-average with exponent in between those corresponding to the geometric and arithmetic averages, and larger than the theoretical value for statistically isotropic media. Yet, assuming that the conductivity is a scalar (as is done when a simple average is used) implies that it is isotropic to flow. At the MADE site there is still enough anisotropic heterogeneity within the blocks to warrant the need of a tensor to describe hydraulic conductivity at the coarse scale. This is why all the Laplacian-based approaches perform better than the simple averaging ones.

Of the Laplacian-based approaches, it is shown that computing tensor conductivities at block centers and then taking the harmonic average of the components corresponding to the directions orthogonal to adjacent interfaces introduces a noise that can be eliminated by aiming directly at upscaling the interblock conductivity tensor to feed directly into the numerical simulator. This is why all interface-centered approaches outperform the block-centered approach.

Of the interblock-centered approaches, analyzing the local flow within an area extending beyond the limits of the block being upscaled (that is, including a skin) also improves the upscaling. The reason being, that the upscaled conductivities are always nonlocal (Neuman and Orr, 1993; Indelman and Abramovich, 1994), that is, they depend not only on the fine-scale conductivities within the block, but on the ones outside, too. Extracting the block to upscale, plus a skin area surrounding it, and applying the boundary conditions of the local flow problems outside the skin, reduces the impact of the boundary conditions inside the block and allows the immediately surrounding fine scale conductivities to impose some control on the flow patterns within the block (as it will happen when the block is embedded in the aquifer). 
The Laplacian-with-skin approach provides a tensor with arbitrary orientation of its principal directions. For the MADE site, it appears that assuming that the principal directions of the block hydraulic conductivity tensors are parallel to the reference axes for all blocks, does not seem to introduce too large an error (compare Figures 9D and 9E), something that could be explained on the basis that the statistical anisotropy model used has its principal directions of continuity aligned with the reference axes for the entire domain. In cases such as cross-bedded formations, or aquifers with a heterogeneity description for which anisotropy varies locally with the domain, the assumption that the principal directions are parallel to the reference axes could not be sustained.

Upscaling induces heterogeneity smoothing, by defining a non-uniform coarse grid that tries to reduce the smoothing on those areas with the highest velocities, and also on areas where fluid velocity will have the largest impact in transport predictions, the results after upscaling will be better than if we define a uniform coarse grid. Although this may appear as a trivial result, it often is disregarded.

But a good reproduction of the fluxes at the coarse scale is not guarantee that transport predictions will be equally good. It has been shown (Fernàndez-Garcia and Gómez-Hernández, 2007; Fernàndez-Garcia et al., 2009; Li et al., 2011) that, in some occasions, after coarsening a hydraulic conductivity grid, the removal of the within-block heterogeneity requires some type of transport upscaling, either modifying the transport parameters (such as enhancing dispersivity) or including transport processes besides advection and dispersion (such as mass transfer). Recall that in our work we kept the same transport equation, with the same parameter values for the fine and coarse scale simulations. But, for the MADE site this is not necessary. The reason is related on how much smearing out of the within-block heterogeneity is induced by the conductivity upscaling. When this smearing out is important, then, there is a need to include other processes; but for the MADE site and the chosen upscaling, this is not the case. The ratio between the coarse block size and the correlation ranges of the fine scale conductivities is substantially smaller than one, in the direction of flow, the ratio is $1 / 8$, in the horizontal plane orthogonal to flow, the ratio is $1 / 3.2$ and in the vertical direction is $1 / 5.5$; this means that the variability of logconductivity within the block is much smaller than the overall variance of 4.5 , and therefore the heterogeneity wiped out by the upscaling process is not as large as to require a further transport upscaling. In the references cited above, the size of the block was on the order of magnitude of the correlation range of the underlying hydraulic conductivity if not larger, and, therefore, upscaling on those cases implied an important smoothing of heterogeneity that had to be taken into account in the transport simulation at the coarse scale.

Can the findings from this work be extrapolated to other case studies? We believe that, regarding flow 
upscaling, yes they can. In fact, the findings from this paper are in agreement with similar works in other case studies. However, regarding transport upscaling, they can be extrapolated only under the same conditions considered here, that is, using coarse blocks smaller than the correlation range, and, using a non-uniform grid with smaller blocks in the areas with highest velocities and in the areas through which the plume will travel.

The final point of discussion is why we have worked trying to reproduce flow and transport on a realization from Salamon et al. (2007) instead of trying to reproduce the available experimental data. This paper did not try to perform a calibration exercise of the MADE site, but rather to help in performing such a calibration in the future. With the work in this paper we show that a coarse scale model, obtained by careful upscaling of a fine scale one, can reproduce the type of transport behavior observed at the MADE site simply using the advection dispersion equation. Trying to calibrate a two-million cell model as obtained by Salamon et al. (2007) is not an easy task, it would require running many times the flow and transport models in many realizations of the site; but those runs would be possible on the coarse models used in our work. The next step in this direction would be to develop a calibration approach that would account for the upscaling step needed to reduce the numerical modeling effort. In its application of such an approach, considering heterogeneity in porosity may also help in obtaining the best calibration; something not needed in our upscaling exercise, since we assume constant porosity attached to the reference conductivity realization.

\section{Summary and Conclusions}

In this paper, we have presented a detailed analysis of the impact of different upscaling techniques on the reproduction of solute transport at the MADE site. We use as a reference a fine scale realization taken from the work by Salamon et al. (2007) that is able to reproduce the contaminant spreading observed in the experiment using an advection-dispersion model. The techniques analyzed span from simple averaging to the estimation of block tensors by local flow models. We have also analyzed the impact that non-uniform coarsening may have in the quality of the results.

This work has three main and important conclusions:

1. In complex environments, such as the MADE site, with hydraulic conductivities which vary over many orders of magnitude, and display an intricate spatial variability, choosing an elaborated upscaling technique yields the best flow and transport results. In particular, the upscaling technique that best performs is the one that computes interblock-centered conductivity tensors using a local solution of the flow equation over a domain including the block plus a skin. 
2. A non-uniform coarsening focused in the refinement of the regions through which the solute plume travels can further improve the results.

3. Modeling of flow and transport at the MADE site has been the object of debate for many years, and many complex transport models have been proposed to reproduce the plume spreading observed. We show that the advection-dispersion model can be used on a coarse model to explain the plume migration in the highly heterogeneous MADE site if careful modeling/upscaling of the flow field is performed, as long as the block size remains smaller than the correlation ranges of the underlying fine scale conductivities. 
Table 1: Variogram parameters for the model fit in Figure 2

\begin{tabular}{ccccc}
\hline Model Type & Sill & \multicolumn{3}{c}{ Range $[\mathrm{m}]$} \\
\cline { 3 - 5 } & $c$ & $a_{x}$ & $a_{y}$ & $a_{z}$ \\
\hline Nugget & 0.424 & & & \\
Spherical & 3.820 & 32 & 80 & 4.1 \\
Hole effect & 0.891 & $\infty$ & 80 & $\infty$ \\
\hline
\end{tabular}




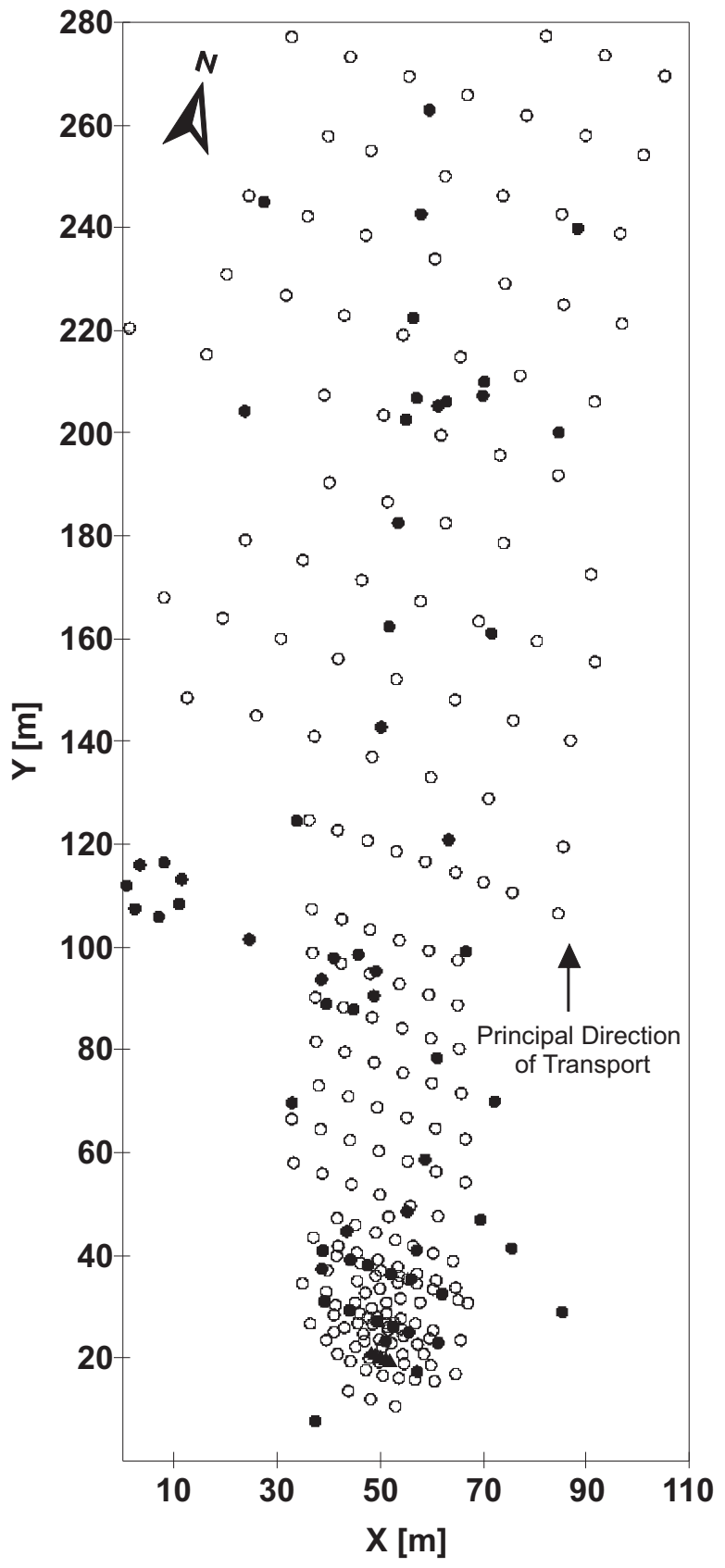

Figure 1: Plan view of model domain. Open circles denote multilevel sampler wells. Triangles indicate the tracer injection wells. Solid circles correspond to flowmeter well locations. 

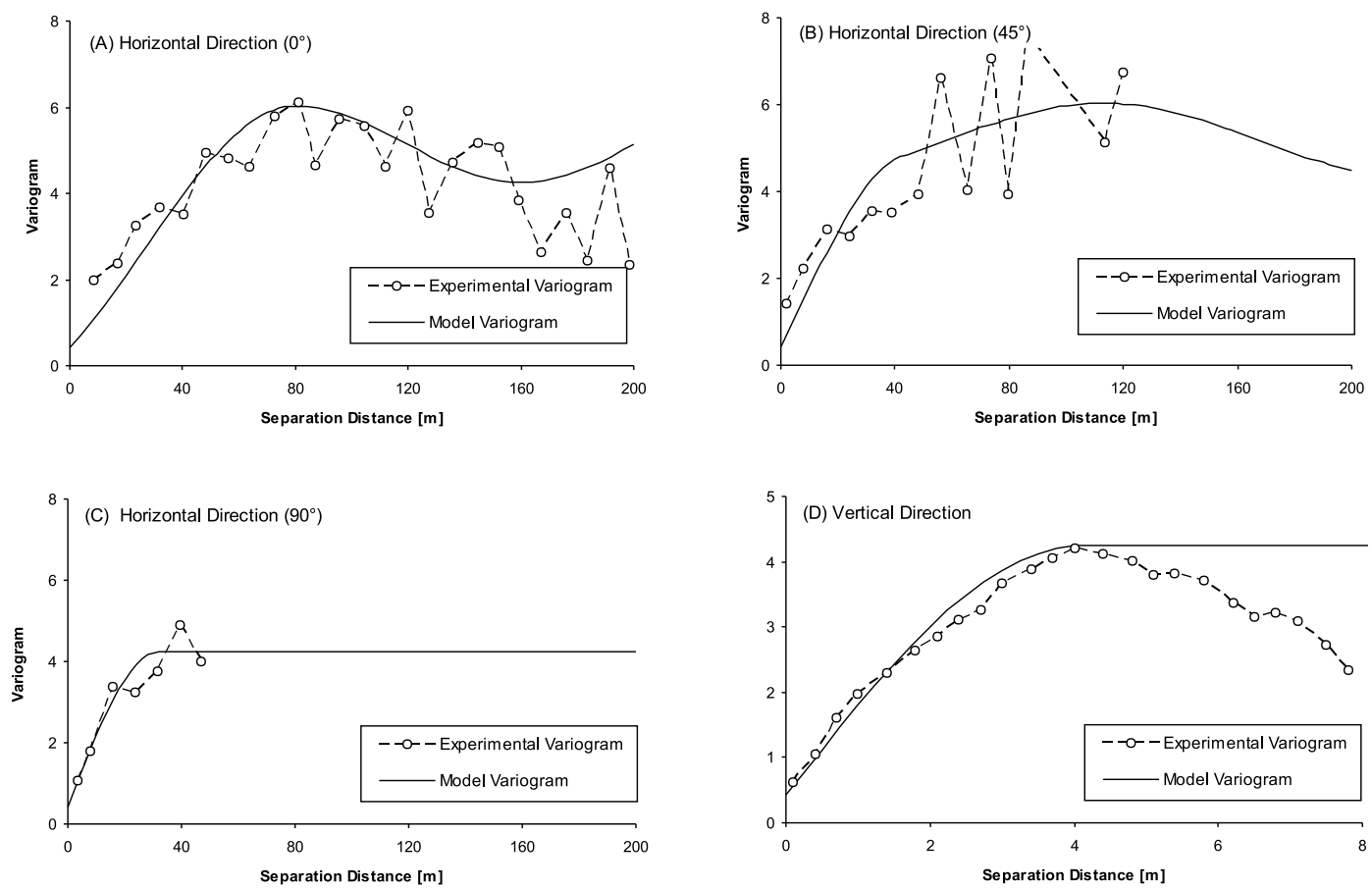

Figure 2: Horizontal and vertical experimental variograms, and fitted model, for the $\ln K$ flowmeter data. The rotation angle of the directional variograms is measured in degrees clockwise from the positive $y$-axis. 


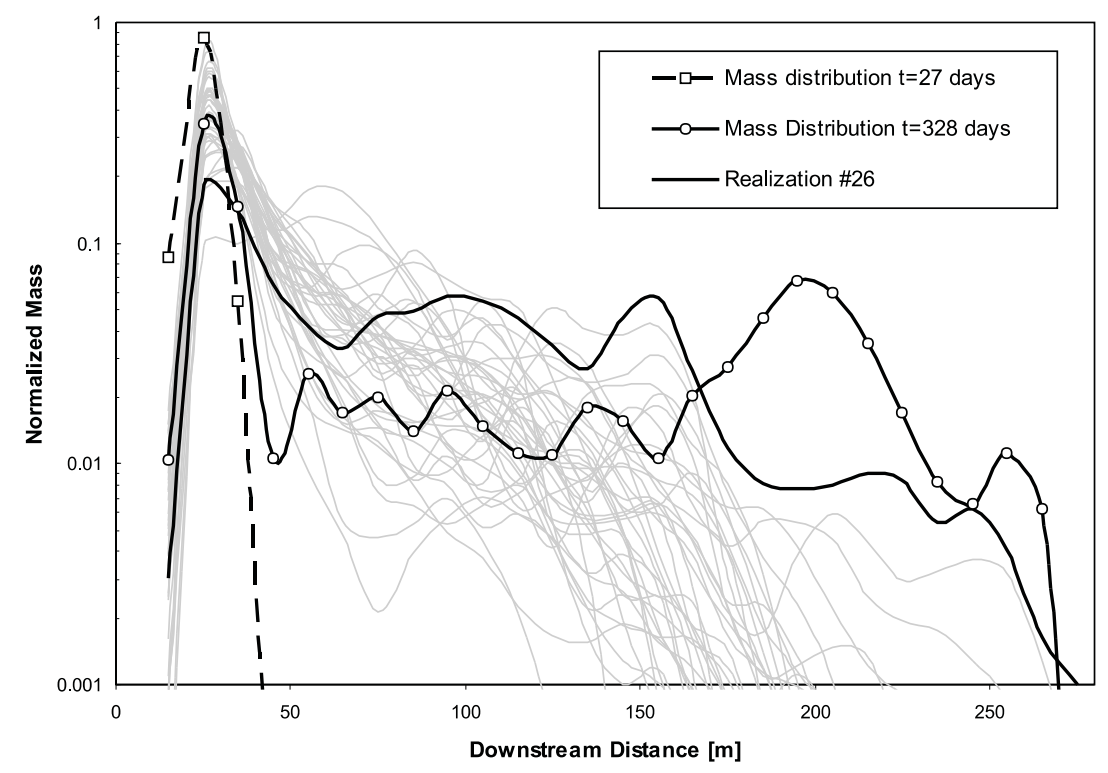

Figure 3: Longitudinal mass distribution profiles of the observed tritium plume at MADE, and predictions on several realizations of hydraulic conductivity. Each realization was generated (on natural-log space) over a grid of $110 \times 280 \times 70$ cells by sequential Gaussian simulation using the variogram model in Equation 1. 


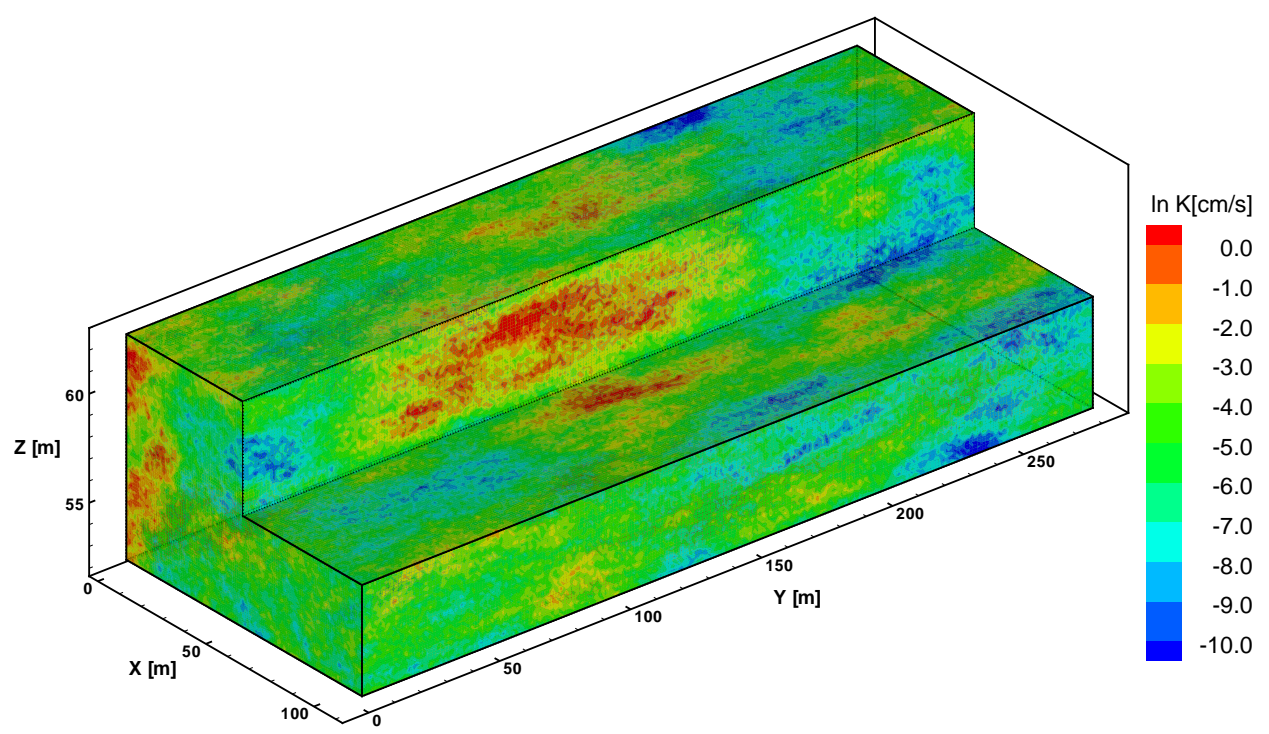

Figure 4: Realization \#26 of $\ln K$ from Salamon et al. (2007). This realization exhibits a strong solute tailing and it is used as the reference in the upscaling exercise. (The scale of the $z$-axis is exaggerated seven times for clarity.)

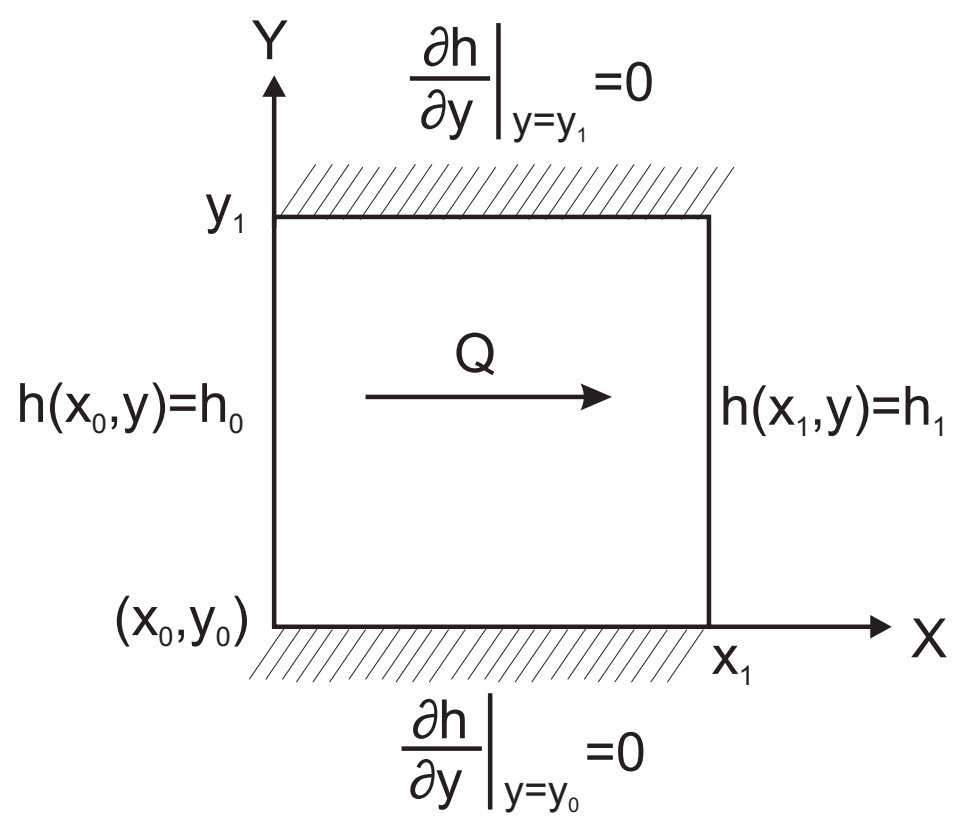

Figure 5: Boundary conditions that would be used in 2D for the local flow model when performing the simple-Laplacian upscaling in order to determine the $x$-component of the hydraulic conductivity tensor. In the simple-Laplacian approach, it is always assumed that the principal directions of the conductivity tensor are parallel to the reference axes. 

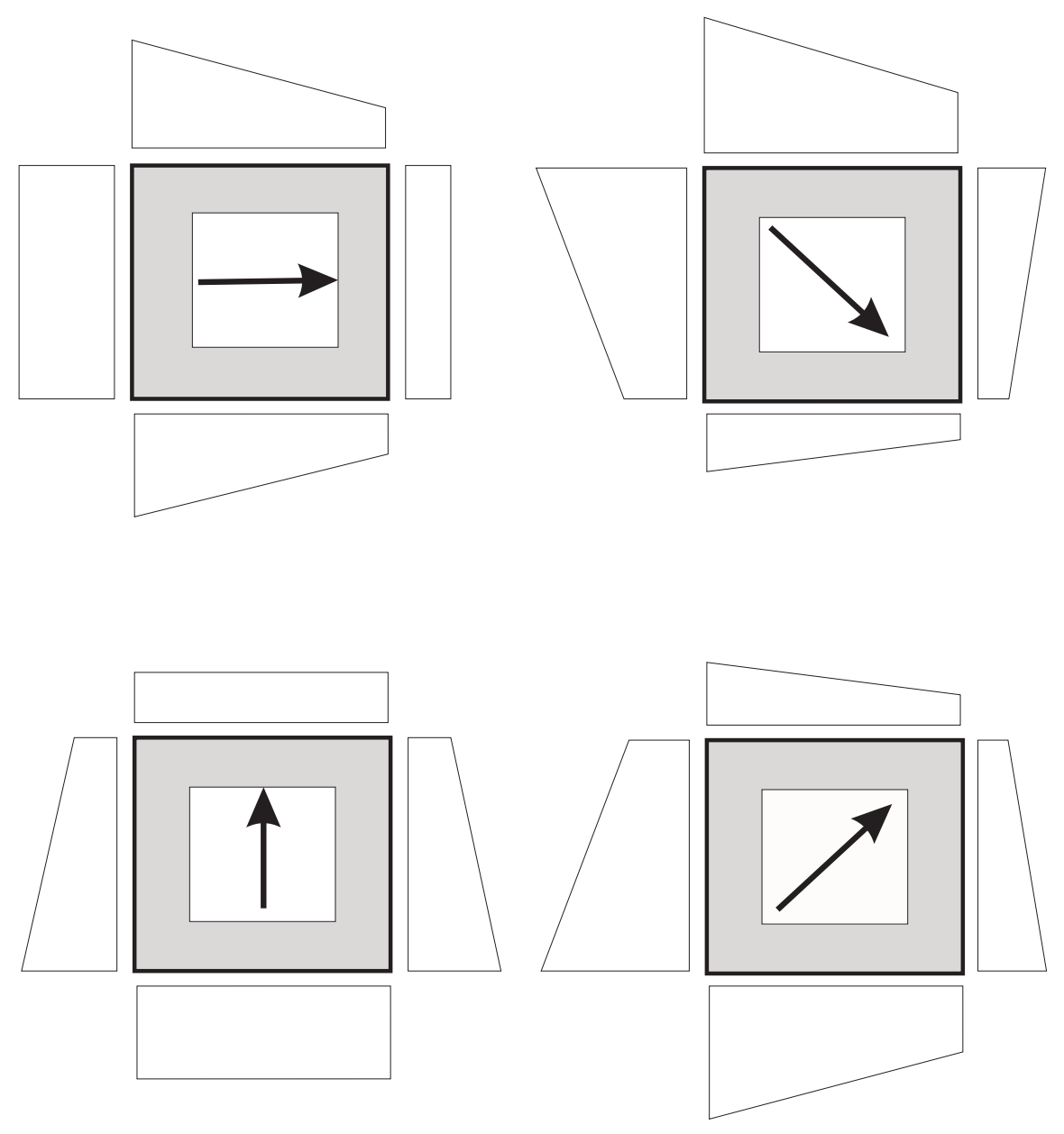

Figure 6: An example of four boundary condition sets that could be used in 2D for the local flow models when performing the Laplacian-with-skin upscaling. The white area is the block being upscaled, and the gray area is the skin region; the arrows indicate the (negative) mean head gradient induced by the prescribed head boundary conditions, and the shapes on the sides of the block indicate the magnitude of the prescribed heads given by tilting planes with gradients opposite to the arrows. 

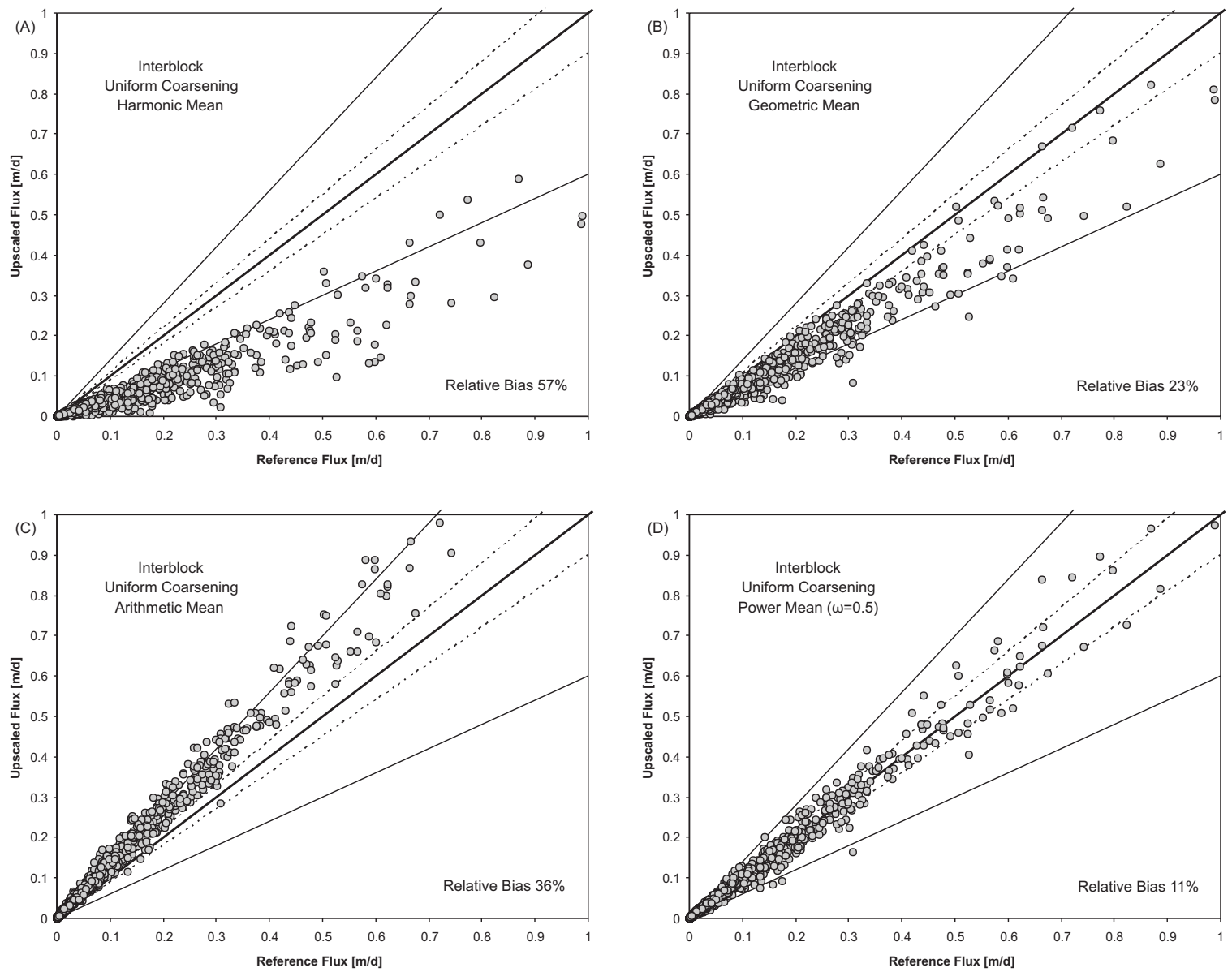

Figure 7: Flow comparison at the fine and coarse scales using simple averaging upscaling approaches. All circles within the dashed lines correspond to coarse scale values that deviate less than $10 \%$ from the reference ones; similarly, all circles within the outer solid lines correspond to coarse scale values that deviate less than $40 \%$. The average relative bias, as defined in Equation 10 , is reported in the lower right corner of each box. 


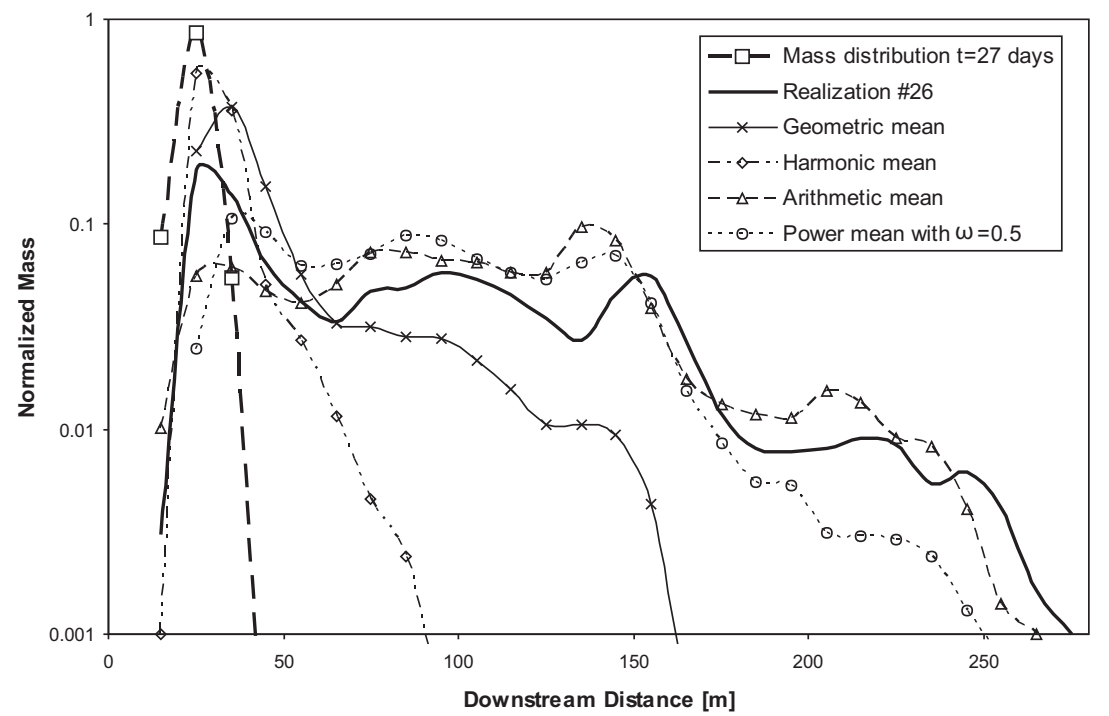

Figure 8: Longitudinal mass distribution profiles of the tritium plume from the fine scale reference realization, and predictions by some simple averaging upscaling approaches at the coarse scale for $t=328$ days. 

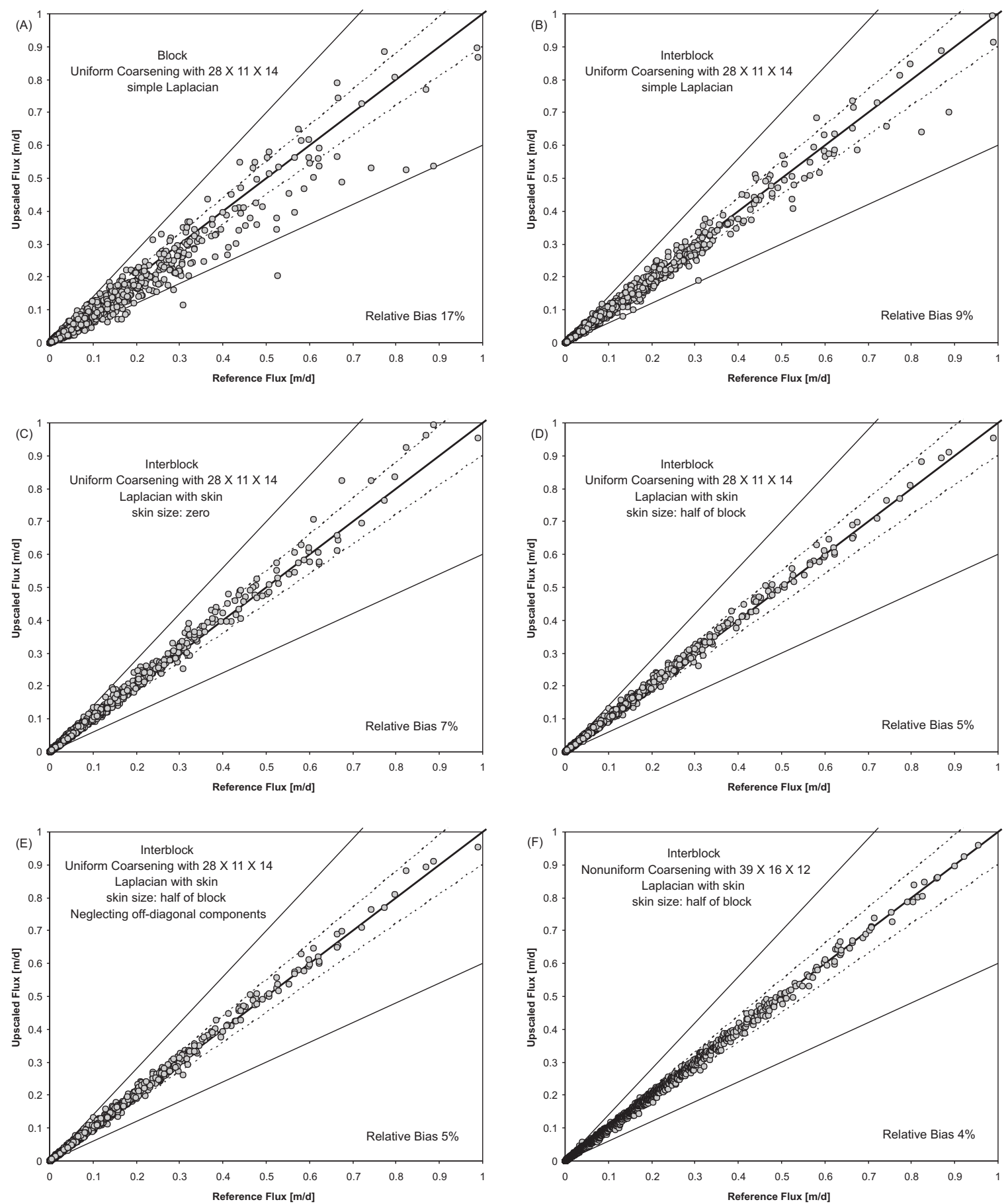

Figure 9: Flow comparison at the fine and coarse scales using Laplacian-based upscaling approaches. All circles within the dashed lines correspond to coarse scale values that deviate less than $10 \%$ from the reference ones; similarly, all circles within the outer solid lines correspond to coarse scale values that deviate less than $40 \%$. The average relative bias, as defined in Equation 10 , is reported in the lower right corner of each box. 


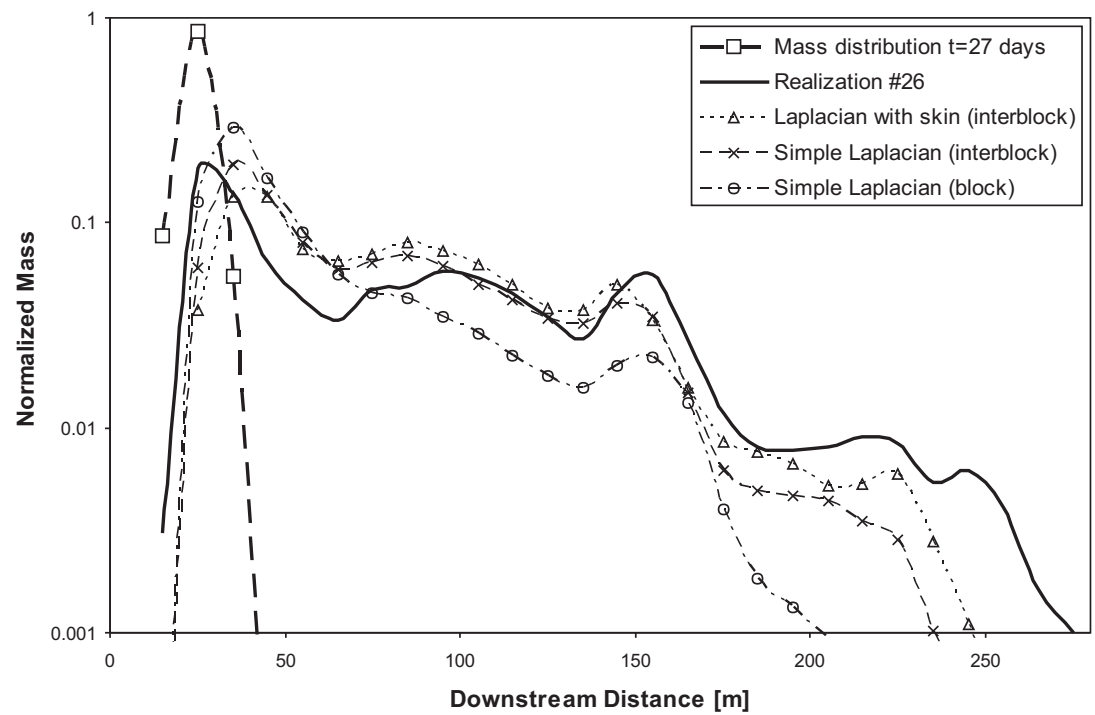

Figure 10: Longitudinal mass distribution profiles of the tritium plume from the fine scale reference realization, and predictions by some Laplacian-based upscaling approaches at the coarse scale, for $t=328$ days.

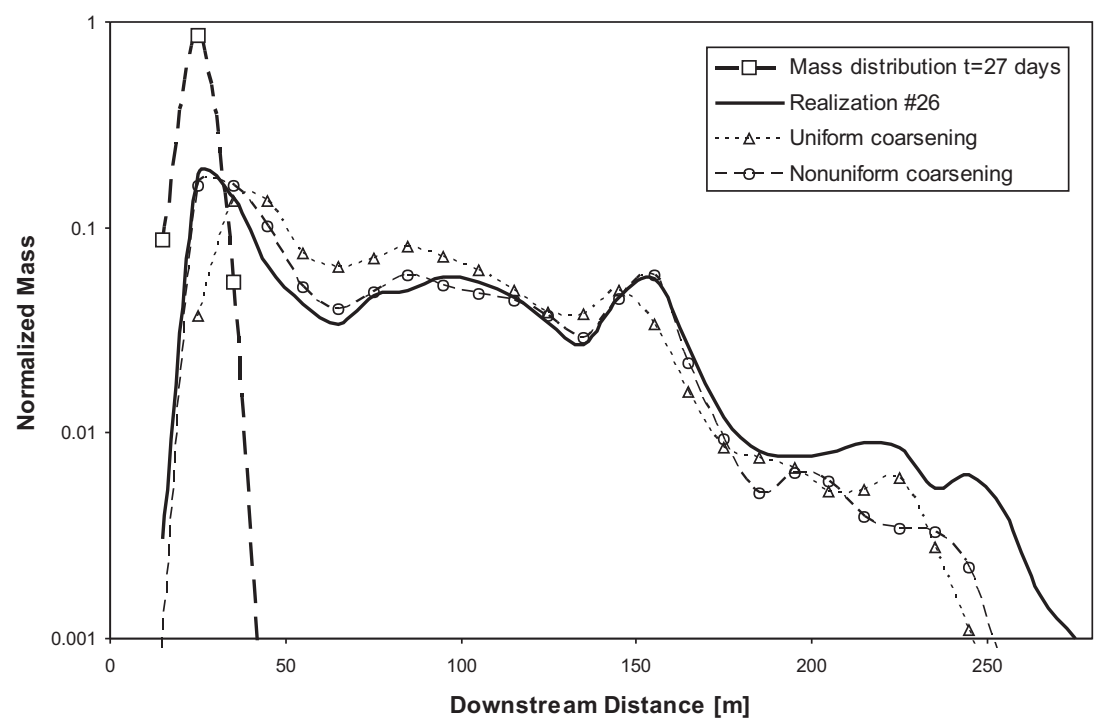

Figure 11: Longitudinal mass distribution profiles of the tritium plume from the fine scale reference realization, and predictions on uniform and non-uniform coarse scale grids, for $t=328$ days. 

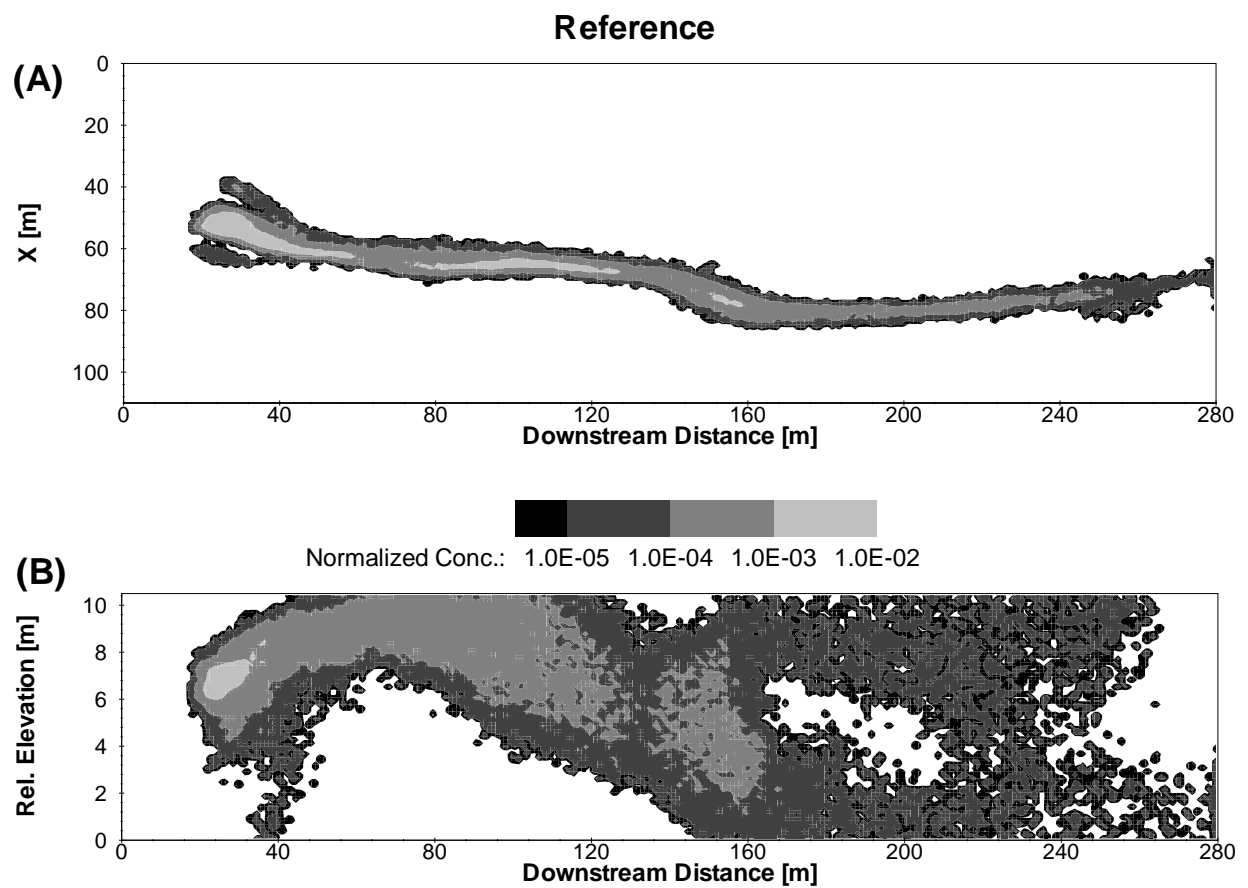

Figure 12: Transport in the fine scale reference realization for $t=328$ days. (A) Depth-integrated normalized concentration distribution. (B) Laterally-integrated normalized concentration distribution. 

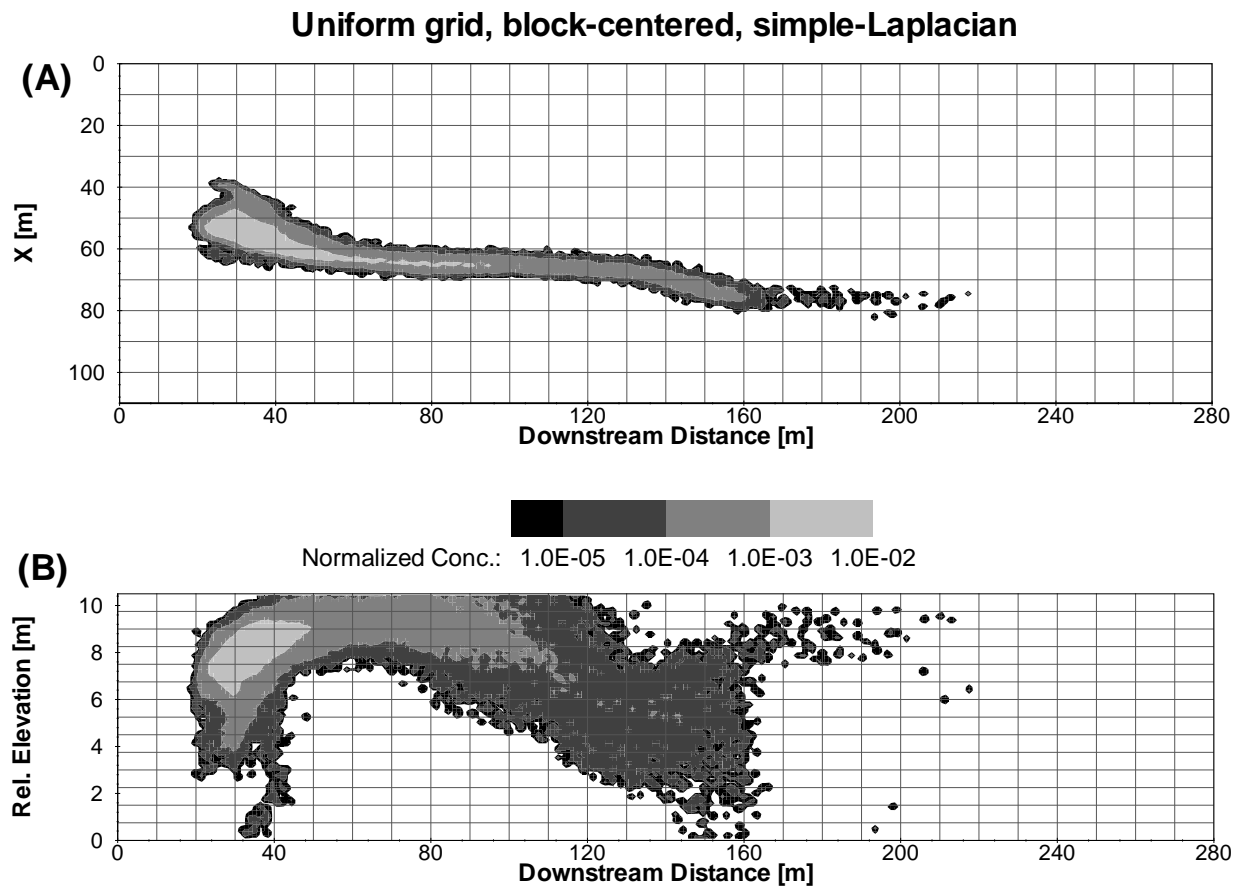

Figure 13: Transport at the coarse scale after upscaling the reference realization on a uniform grid using a block-centered simpleLaplacian approach for $t=328$ days. (A) Depth-integrated normalized concentration distribution. (B) Laterally-integrated normalized concentration distribution. 

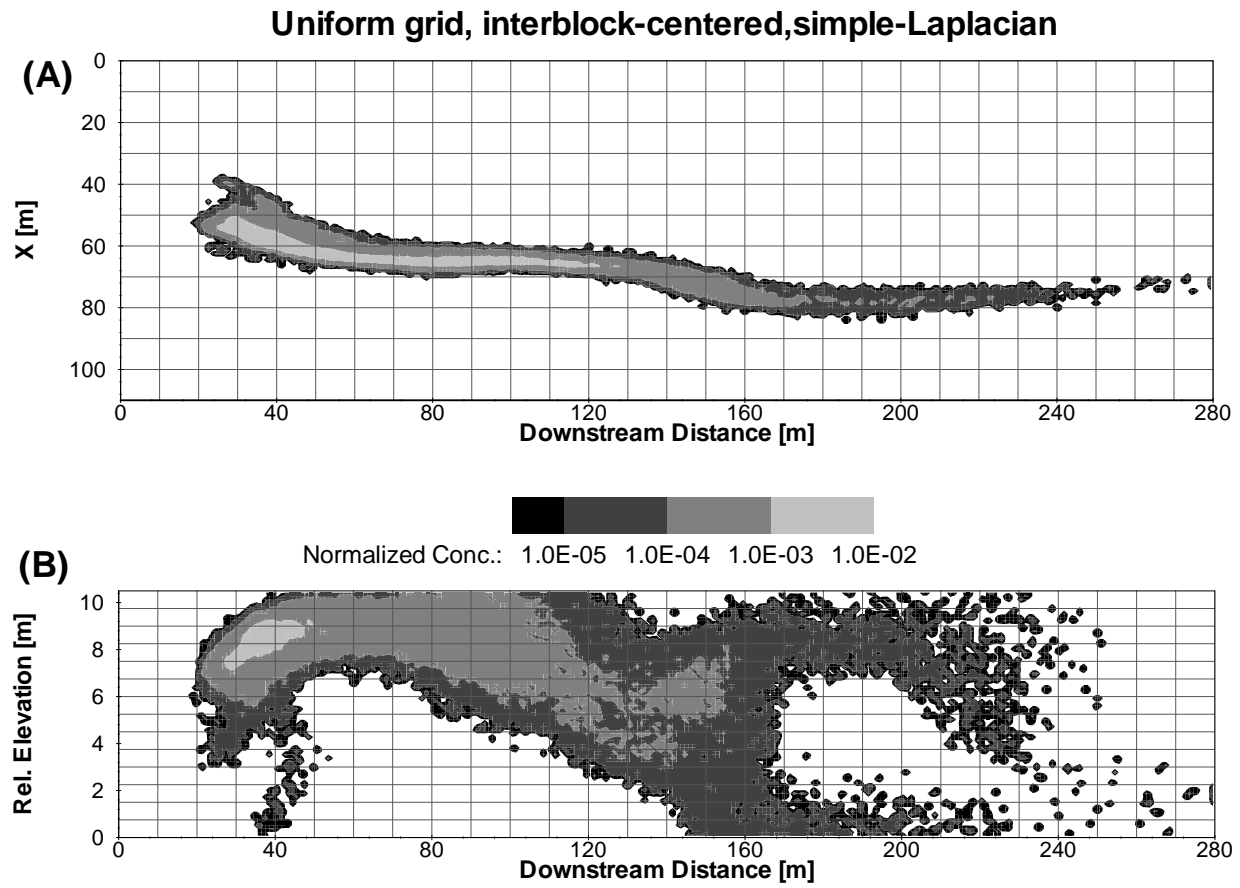

Figure 14: Transport at the coarse scale after upscaling the reference realization on a uniform grid using an interblock-centered simple-Laplacian approach for $t=328$ days. (A) Depth-integrated normalized concentration distribution. (B) Laterallyintegrated normalized concentration distribution. 

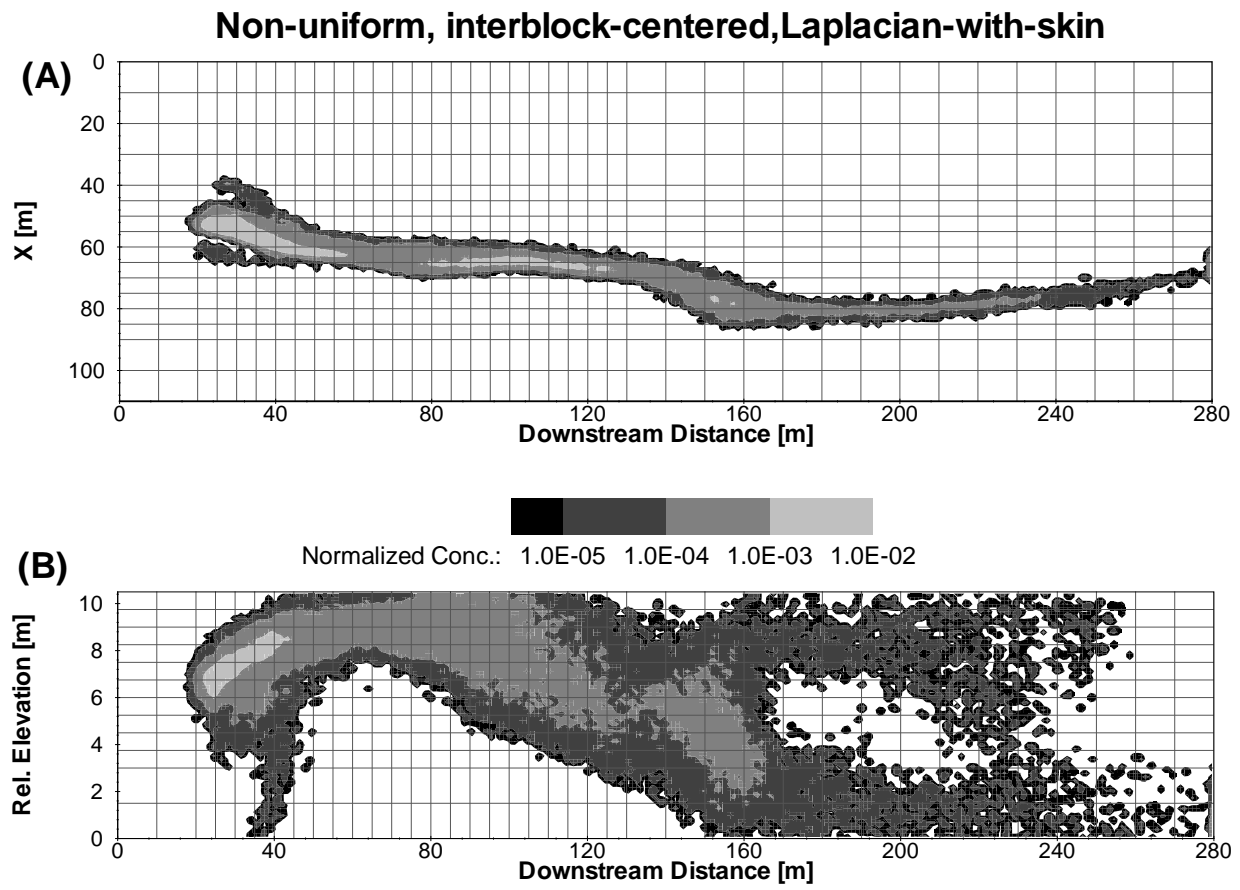

Figure 15: Transport at the coarse scale after upscaling the reference realization on a non-uniform grid using an interblockcentered Laplacian-with-skin approach for $t=328$ days.

(A) Depth-integrated normalized concentration distribution. (B) Laterally-integrated normalized concentration distribution. 


\section{Appendix A}

A nineteen-point block-centered finite-difference procedure for the solution of saturated groundwater steady flow in 3D with full tensor conductivities is described here. In the absence of sinks and sources, the partial differential equation governing flow in three-dimensions can be expressed as:

$\frac{\partial}{\partial x}\left(K_{x x} \frac{\partial h}{\partial x}+K_{x y} \frac{\partial h}{\partial y}+K_{x z} \frac{\partial h}{\partial z}\right)+\frac{\partial}{\partial y}\left(K_{x y} \frac{\partial h}{\partial x}+K_{y y} \frac{\partial h}{\partial y}+K_{y z} \frac{\partial h}{\partial z}\right)+\frac{\partial}{\partial z}\left(K_{x z} \frac{\partial h}{\partial x}+K_{y z} \frac{\partial h}{\partial y}+K_{z z} \frac{\partial h}{\partial z}\right)=0$

If this equation is discretized with a nineteen-point block-centered finite-difference stencil over a non-uniform grid of parallelpipedal blocks, the following equation results for a generic block $(i, j, k)$ of size $\left.\Delta x\right|_{i, j, k} \times$ $\left.\Delta y\right|_{i, j, k} \times\left.\Delta z\right|_{i, j, k}$ (see Figure A-1):

$$
\begin{aligned}
& \frac{1}{\left.\Delta x\right|_{i, j, k}}\left[\left.\left(K_{x x} \frac{\partial h}{\partial x}+K_{x y} \frac{\partial h}{\partial y}+K_{x z} \frac{\partial h}{\partial z}\right)\right|_{i+1 / 2, j, k}-\left.\left(K_{x x} \frac{\partial h}{\partial x}+K_{x y} \frac{\partial h}{\partial y}+K_{x z} \frac{\partial h}{\partial z}\right)\right|_{i-1 / 2, j, k}\right]+ \\
& \frac{1}{\left.\Delta y\right|_{i, j, k}}\left[\left.\left(K_{x y} \frac{\partial h}{\partial x}+K_{y y} \frac{\partial h}{\partial y}+K_{y z} \frac{\partial h}{\partial z}\right)\right|_{i, j+1 / 2, k}-\left.\left(K_{x y} \frac{\partial h}{\partial x}+K_{y y} \frac{\partial h}{\partial y}+K_{y z} \frac{\partial h}{\partial z}\right)\right|_{i, j-1 / 2, k}\right]+ \\
& \frac{1}{\left.\Delta z\right|_{i, j, k}}\left[\left.\left(K_{x z} \frac{\partial h}{\partial x}+K_{y z} \frac{\partial h}{\partial y}+K_{z z} \frac{\partial h}{\partial z}\right)\right|_{i, j, k+1 / 2}-\left.\left(K_{x z} \frac{\partial h}{\partial x}+K_{y z} \frac{\partial h}{\partial y}+K_{z z} \frac{\partial h}{\partial z}\right)\right|_{i, j, k-1 / 2}\right]=0
\end{aligned}
$$

The hydraulic gradients at the interfaces are approximated by central differences from the heads at the nineteen blocks surrounding $(i, j, k)$, That is,

$$
\begin{aligned}
\left.\frac{\partial h}{\partial x}\right|_{i+1 / 2, j, k} & =\frac{h_{i, j+1, k}-h_{i, j-1, k}}{\left.\triangle x\right|_{i, j+1, k}+\left.2 \triangle x\right|_{i, j, k}+\left.\triangle x\right|_{i, j-1, k}}+\frac{h_{i+1, j+1, k}-h_{i+1, j-1, k}}{\left.\triangle x\right|_{i+1, j+1, k}+\left.2 \triangle x\right|_{i+1, j, k}+\left.\triangle x\right|_{i+1, j-1, k}} \\
\left.\frac{\partial h}{\partial y}\right|_{i+1 / 2, j, k} & =\frac{2\left(h_{i+1, j, k}-h_{i, j, k}\right)}{\left.\triangle y\right|_{i+1, j, k}+\left.\triangle y\right|_{i, j, k}} \\
\left.\frac{\partial h}{\partial z}\right|_{i+1 / 2, j, k} & =\frac{h_{i, j, k+1}-h_{i, j, k-1}}{\left.\triangle z\right|_{i, j, k+1}+\left.2 \triangle z\right|_{i, j, k}+\left.\triangle z\right|_{i, j, k-1}}+\frac{h_{i+1, j, k+1}-h_{i+1, j, k-1}}{\left.\triangle z\right|_{i+1, j, k+1}+\left.2 \triangle z\right|_{i+1, j, k}+\left.\triangle z\right|_{i+1, j, k-1}}
\end{aligned}
$$

The partial derivatives of the hydraulic head in the other five interfaces can be given by similar expressions. Substituting (A-3) into (A-2), multiplying both sides by $\left.\left.\left.\Delta x\right|_{i, j, k} \Delta y\right|_{i, j, k} \Delta z\right|_{i, j, k}$, and rearranging terms, the 


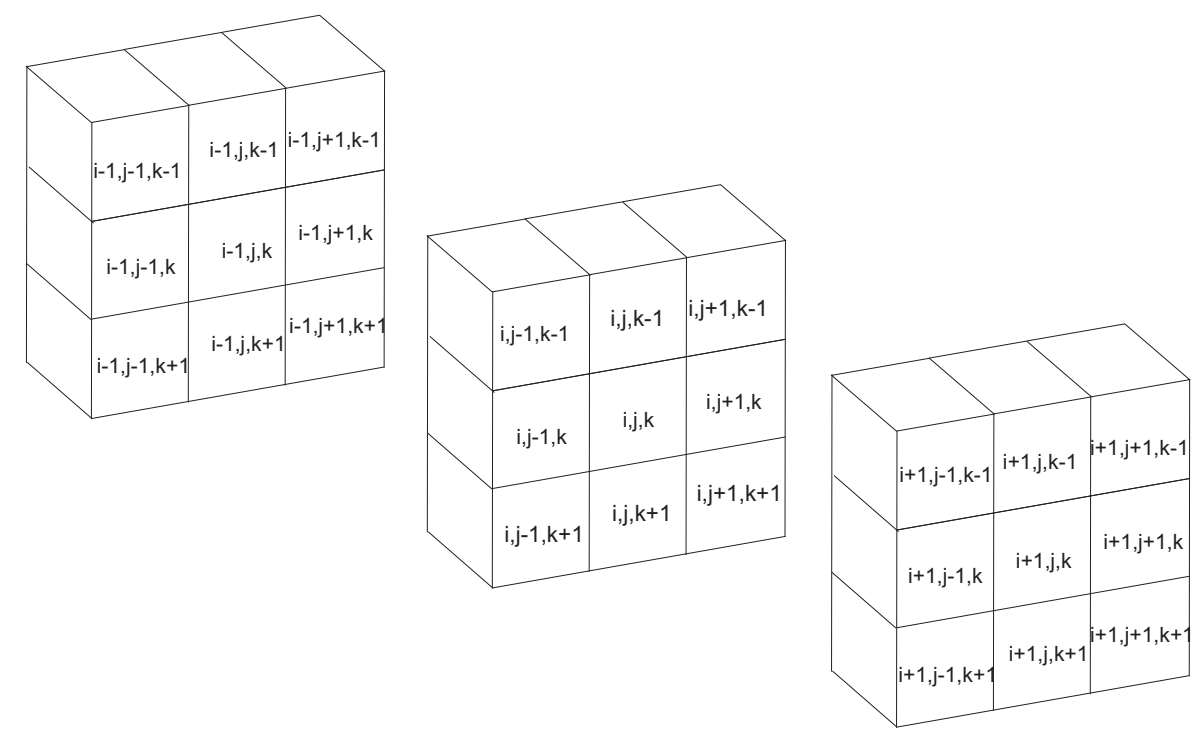

Figure A-1: Schematic illustration of the 3D finite-difference spatial discretization

nineteen-point results in:

$$
\begin{aligned}
& A h_{i, j+1, k}+B h_{i, j, k}+C h_{i+1, j+1, k}+D h_{i-1, j+1, k}+E h_{i+1, j, k}+F h_{i-1, j, k}+G h_{i, j+1, k+1}+ \\
& H h_{i, j+1, k-1}+I h_{i, j, k+1}+J h_{i, j, k-1}+K h_{i, j-1, k}+L h_{i+1, j-1, k}+M h_{i-1, j-1, k}+ \\
& N h_{i, j-1, k+1}+O h_{i, j-1, k-1}+P h_{i+1, j, k+1}+Q h_{i+1, j, k-1}+R h_{i-1, j, k+1}+S h_{i-1, j, k-1}=0
\end{aligned}
$$

where A, B, ..., S are function of the block sizes and interface hydraulic conductivity components. Equation (A-4) is written for all the nodes within the aquifer, except for those for which head is prescribed, resulting in a set of linear equations.

Acknowledgements The authors gratefully acknowledge the financial support by ENRESA (project 0079000029). The second author also acknowledges the financial support from China Scholarship Council. The two anonymous reviewers are gratefully acknowledged for their comments which helped improving the final version of the manuscript. 


\section{References}

Adams, E. E., Gelhar, L. W., 1992. Field study of dispersion in a heterogeneous aquifer 2. spatial moments analysis. Water Resources Research 28 (12), 3293-3307.

Anderman, E. R., Kipp, K. L., Hill, M. C., Valstar, J., Neupauer, R. M., 2002. MODFLOW-2000, the US geological survey modular Ground-Water Model Documentation of the Model-Layer Variable-Direction horizontal anisotropy (LVDA) capability of the Hydrogeologic-Unit flow (HUF) package. US Geological Survey, Open file Report, 02-409.

Appel, C. A., 1976. A note on computing finite difference interblock transmissivities. Water Resources Research $12(3), 561-563$.

Bachu, S., Cuthiell, D., 1990. Effects of Core-Scale heterogeneity on steady state and transient fluid flow in porous media: Numerical analysis. Water Resources Research 26 (5), 863-874.

Baeumer, B., Benson, D. A., Meerschaert, M. M., Wheatcraft, S. W., 2001. Subordinated advectiondispersion equation for contaminant transport. Water Resources Research 37 (6), 1543-1550.

Barlebo, H. C., Hill, M. C., Rosbjerg, D., 2004. Investigating the macrodispersion experiment (MADE) site in columbus, mississippi, using a three-dimensional inverse flow and transport model. Water Resources Research 40 (4), W04211.

Bear, J., 1972. Dynamics of fluids in porous media. American Elsevier Pub. Co., New York.

Begg, S. H., Carter, R. B. R. C., Dranfield, P. B. A., 1989. Assigning effective values to simulator gridblock parameters for heterogeneous reservoirs. SPE (Society of Petroleum Engineers) Reservoir Engineering.

Benson, D. A., Schumer, R., Meerschaert, M. M., Wheatcraft, S. W., 2001. Fractional dispersion, levy motion, and the MADE tracer tests. Transport in porous media 42 (1), 211-240.

Berkowitz, B., Scher, H., 1998. Theory of anomalous chemical transport in random fracture networks. Physical Review E 57 (5), 5858-5869.

Bierkens, M. F. P., Weerts, H. J. T., 1994. Block hydraulic conductivity of cross-bedded fluvial sediments. Water Resources Research 30 (10), 2665-2678. 
Boggs, J., Beard, L., Waldrop, W., Stauffer, T., MacIntyre, W., Antworth, C., 1993. Transport of tritium and four organic compounds during a natural gradient experiment (MADE-2), EPRI Report TR-101998. Electric Power Research Institute, Palo Alto, CA 94304.

Boggs, J. M., Adams, E. E., 1992. Field study of dispersion in a heterogeneous aquifer 4. investigation of adsorption and sampling bias. Water Resources Research 28 (12), 3325-3336.

Boggs, J. M., Young, S. C., Beard, L. M., Gelhar, L. W., Rehfeldt, K. R., Adams, E. E., 1992. Field study of dispersion in a heterogeneous aquifer 1. overview and site description. Water Resources Research 28 (12), $3281-3291$.

Bourgeat, A., 1984. Homogenized behavior of two-phase flows in naturally fractured reservoirs with uniform fractures distribution. Computer Methods in Applied Mechanics and Engineering 47 (1-2), 205-216.

Bouwer, H., 1969. Planning and interpreting soil permeability measurements. Journal of the Irrigation and Drainage Division, ASCE 95, 391-402.

Cardwell, W. T., Parsons, R. L., 1945. Averaging permeability of heterogeneous oil sands. Transactions of the American Institute of Mining, Metallurgical and Petroleum Engineers 160, 34-42.

Chen, Y., Durlofsky, L. J., Gerritsen, M., Wen, X. H., 2003. A coupled local-global upscaling approach for simulating flow in highly heterogeneous formations. Advances in Water Resources 26 (10), 1041-1060.

Desbarats, A. J., 1987. Numerical estimation of effective permeability in Sand-Shale formations. Water Resources Research 23 (2), 273-286.

Desbarats, A. J., 1988. Estimation of effective permeabilities in the lower stevens formation of the paloma field, san joaquin valley, california. SPE Reservoir Engineering 3 (4), 1301-1307.

Desbarats, A. J., 1992. Spatial averaging of hydraulic conductivity in three-dimensional heterogeneous porous media. Mathematical Geology 24 (3), 249-267.

Deutsch, C. V., 1989. Calculating effective absolute permeability in Sand-Shale sequences. SPE Formation Evaluation 4 (3), 343-348.

Durlofsky, L. J., 1991. Numerical calculations of equivalent grid block permeability tensors for heterogeneous porous media. Water Resources Research 27 (5), 699-708. 
Durlofsky, L. J., Jones, R. C., Milliken, W. J., 1997. A nonuniform coarsening approach for the scale-up of displacement processes in heerogeneous porous media. Advances in Water Resources 20 (5-6), 335-347.

Eggleston, J., Rojstaczer, S., 1998. Identification of Large-Scale hydraulic conductivity trends and the influence of trends on contaminant transport. Water Resources Research 34 (9), 2155-2168.

Feehley, C. E., Zheng, C., Molz, F. J., 2000. A dual-domain mass transfer approach for modeling solute transport in heterogeneous aquifers: Application to the macrodispersion experiment (MADE) site. Water Resources Research 36 (9), 2501-2515.

Fernàndez-Garcia, D., Gómez-Hernández, J. J., 2007. Impact of upscaling on solute transport: Traveltimes, scale dependence of dispersivity, and propagation of uncertainty. Water Resources Research 43 (2).

Fernàndez-Garcia, D., Illangasekare, T. H., Rajaram, H., 2005. Differences in the scale dependence of dispersivity and retardation factors estimated from forced-gradient and uniform flow tracer tests in threedimensional physically and chemically heterogeneous porous media. Water Resources Research 41 (3), W03012.

Fernàndez-Garcia, D., Llerar-Meza, G., Gómez-Hernández, J. J., 2009. Upscaling transport with mass transfer models: Mean behavior and propagation of uncertainty. Water Resources Research 45, W10411.

Flodin, E. A., Durlofsky, L. J., Aydin, A., 2004. Upscaled models of flow and transport in faulted sandstone: boundary condition effects and explicit fracture modelling. Petroleum Geoscience 10 (2), 173-181.

Freeze, R. A., Cherry, J. A., 1979. Groundwater. Prentice-Hall.

Garcia, M., Journel, A. G., Aziz, K., 1992. Automatic grid generation for modeling reservoir heterogeneities. SPE Reservoir Engineering 1992, 278-284.

Gillham, R. W., Robin, M. J. L., Dytynyshyn, D. J., Johnston, H. M., 1984. Diffusion of nonreactive and reactive solutes through fine-grained barrier materials. Canadian Geotechnical Journal 21 (3), 541-550.

Gómez-Hernández, J. J., 1991. A stochastic approach to the simulation of block conductivity values conditioned upon data measured at a smaller scale. Ph.D. thesis, Stanford University.

Gómez-Hernández, J. J., Journel, A. G., 1993. Joint sequential simulation of multi-Gaussian fields. Geostatistics Troia $92(1), 85-94$. 
Gómez-Hernández, J. J., Wen, X. H., 1994. Probabilistic assessment of travel times in groundwater modeling. J. of Stochastic Hydrology and Hydraulics 8 (1), 19-56.

Guan, J., Molz, F. J., Zhou, Q., Liu, H. H., Zheng, C., 2008. Behavior of the mass transfer coefficient during the MADE-2 experiment: New insights. Water Resources Research 44, W02423.

Harbaugh, A. W., Banta, E. R., Hill, M. C., McDonald, M. G., 2000. MODFLOW-2000, the U.S. Geological Survey modular ground-water model. English. U.S. Geological Survey, Branch of Information Services, Reston, VA, Denver, CO.

Harvey, C., Gorelick, S. M., 2000. Rate-Limited mass transfer or macrodispersion: Which dominates plume evolution at the macrodispersion experiment (MADE) site? Water Resources Research 36 (3), 637-650.

Hill, M. C., 1990. Preconditioned conjugate gradient 2 (PCG2). A computer program for solving groundwater flow equations: US Geological Survey Water-Resources Investigations Report, 98-4048.

Hill, M. C., Barlebo, H. C., Rosbjerg, D., 2006. Reply to comment by F. Molz et al. on investigating the macrodispersion experiment (MADE) site in columbus, mississippi, using a three-dimensional inverse flow and transport model. Water Resources Research 42 (6), W06604.

Indelman, P., Abramovich, B., 1994. Nonlocal properties of nonuniform averaged flows in heterogeneous media. Water Resour. Res. 30 (12), 3385-3393.

URL http://dx.doi.org/10.1029/94WR01782

Jourde, H., Flodin, E. A., Aydin, A., Durlofsky, L. J., Wen, X. H., 2002. Computing permeability of fault zones in eolian sandstone from outcrop measurements. AAPG bulletin 86 (7), 1187-1200.

Journel, A. G., Deutsch, C. V., Desbarats, A. J., 1986. Power averaging for block effective permeability. SPE 15128.

LaBolle, E. M., Fogg, G. E., Tompson, A. F., 1996. Random-walk simulation of transport in heterogeneous porous media: Local mass-conservation problem and implementation methods. Water Resources Research $32(3), 583-593$.

Lake, L. W., 1988. The origins of anisotropy. J. of Petr. Techn. April, 395-396.

Li, L., Zhou, H., Gómez-Hernández, J. J., 2010. Steady-state groundwater flow modeling with full tensor conductivities using finite differences. Computers \& Geosciences 36 (10), 1211-1223. 
Li, L., Zhou, H., Gómez-Hernández, J. J., 2011. Transport upscaling using multi-rate mass transfer in three-dimensional highly heterogeneous porous media. Advances in Water Resources, in press, doi:10.1016/j.advwatres.2011.01.001.

Liu, G., Chen, Y., Zhang, D., 2008. Investigation of flow and transport processes at the MADE site using ensemble kalman filter. Advances in Water Resources 31 (7), 975-986.

Llopis-Albert, C., Capilla, J. E., 2009. Gradual conditioning of non-Gaussian transmissivity fields to flow and mass transport data: 3. application to the macrodispersion experiment (MADE-2) site, on Columbus Air Force Base in Mississippi (USA). Journal of Hydrology 371 (1-4), 75-84.

Matheron, G., 1967. Elements pour une theorie des milieux poreux. Masson et Cie.

Molz, F. J., Zheng, C., Gorelick, S. M., Harvey, C. F., 2006. Comment on Investigating the macrodispersion experiment (MADE) site in columbus, mississippi, using a three-dimensional inverse flow and transport model by Heidi Christiansen Barlebo, Mary C. Hill, and Dan Rosbjerg. Water Resources Research 42 (6), W06603.

Neuman, S. P., Orr, S., 1993. Prediction of steady state flow in nonuniform geologic media by conditional moments: Exact nonlocal formalism, effective conductivities, and weak approximation. Water Resour. Res. 29 (2), 341-364.

Press, W. H., Flannery, B. P., Teukolsky, S. A., Vetterling, W. T., 1988. Numerical recipes in C. Cambridge University Press, Cambridge.

Rehfeldt, K. R., Boggs, J. M., Gelhar, L. W., 1992. Field study of dispersion in a heterogeneous aquifer 3. geostatistical analysis of hydraulic conductivity. Water Resources Research 28 (12), 3309-3324.

Renard, P., Marsily, G. D., 1997. Calculating equivalent permeability: A review. Advances in Water Resources $20(5-6), 253-278$.

Romeu, R. K., Noetinger, B., 1995. Calculation of internodal transmissivities in finite difference models of flow in heterogeneous porous media. Water Resources Research 31 (4), 943-959.

Salamon, P., Fernàndez-Garcia, D., Gómez-Hernández, J. J., 2006. A review and numerical assessment of the random walk particle tracking method. Journal of Contaminant Hydrology 87 (3-4), 277-305. 
Salamon, P., Fernàndez-Garcia, D., Gómez-Hernández, J. J., 2007. Modeling tracer transport at the MADE site: the importance of heterogeneity. Water Resources Research 30 (8).

Sánchez-Vila, X., Carrera, J., Girardi, J. P., 1996. Scale effects in transmissivity. Journal of Hydrology $183(1-2), 1-22$.

Sánchez-Vila, X., Guadagnini, A., Carrera, J., 2006. Representative hydraulic conductivities in saturated groundwater flow. Reviews of Geophysics 44 (3).

Schumer, R., Benson, D. A., Meerschaert, M. M., Baeumer, B., 2003. Fractal mobile/immobile solute transport. Water Resources Research 39 (10), 1296.

Vermeulen, P. T. M., Stroet, C. B. M. T., Heemink, A. W., 2006. Limitations to upscaling of groundwater flow models dominated by surface water interaction. Water Resources Research 42 (10), W10406.

Warren, J. E., Price, H. S., 1961. Flow in heterogeneous porous media. Society of Petroleum Engineering Journal 1, 153-169.

Wen, X. H., Durlofsky, L. J., Edwards, M., 2003. Use of border regions for improved permeability upscaling. Mathematical Geology 35 (5), 521-547.

Wen, X. H., Gómez-Hernández, J. J., 1996a. The constant displacement scheme for tracking particles in heterogeneous aquifers. Groundwater 34 (1), 135-142.

Wen, X. H., Gómez-Hernández, J. J., 1996b. Upscaling hydraulic conductivities: An overview. Journal of Hydrology 183 (1-2), ix-xxxii.

Wen, X. H., Gómez-Hernández, J. J., 1998. Upscaling conductivities in cross-bedded formations. Mathematical Geology 30 (2), 181-212.

Zhou, H., Li, L., Gómez-Hernández, J. J., 2010. Three-dimensional hydraulic conductivity upscaling in groundwater modelling. Computers \& Geosciences 36 (10), 1224-1235. 


\title{
A Comparative Study of Three-Dimensional Hydraulic Conductivity Upscaling at the MAcro-Dispersion Experiment (MADE) site, Columbus Air Force Base, Mississippi (USA)
}

\author{
Liangping Li ${ }^{a, *}$, Haiyan Zhou ${ }^{a}$, J. Jaime Gómez-Hernández ${ }^{\mathrm{a}}$ \\ ${ }^{a}$ Group of Hydrogeology, Universitat Politècnica de València, Camino de Vera, s/n, 46022 Valencia, Spain
}

\begin{abstract}
Simple averaging, simple-Laplacian, Laplacian-with-skin, and non-uniform coarsening are the techniques investigated in this comparative study of three-dimensional hydraulic conductivity upscaling. The reference is a fine scale conditional realization of the hydraulic conductivities at the MAcro-Dispersion Experiment site on Columbus Air Force Base in Mississippi (USA). This realization was generated using a hole-effect variogram model and it was shown that flow and transport modeling in this realization (at this scale) can reproduce the observed non-Fickian spreading of the tritium plume. The purpose of this work is twofold, first to compare the effectiveness of different upscaling techniques in yielding upscaled models able to reproduce the observed transport behavior, and second to demonstrate and analyze the conditions under which flow upscaling can provide a coarse model in which the standard advection-dispersion equation can be used to model transport in seemingly non-Fickian scenarios. Specifically, the use of the Laplacian-with-skin upscaling technique coupled with a non-uniform coarsening scheme yields the best results both in terms of flow and transport reproduction, for this case study in which the coarse blocks are smaller than the correlation ranges of the fine scale conductivities.

Keywords: full tensor, upscaling, interblock, non-uniform coarsening, MADE site, non-Fickian behavior
\end{abstract}

\section{1. Introduction}

In the last decades, two large-scale natural-gradient tracer tests were conducted to enhance the understanding of solute transport in highly heterogenous aquifers. These experiments were conducted at the Columbus Air Force Base in Mississippi, where the hydraulic conductivity variability is very high, with $\sigma_{\ln K}^{2} \approx 4.5$ (Rehfeldt et al., 1992). The site and the experiments performed are commonly referred to as

\footnotetext{
*Corresponding author. Tel: +34 963879615 Fax: +34 963879492

Email addresses: liali@upvnet.upv.es (Liangping Li), haizh@upvnet.upv.es (Haiyan Zhou), jaime@dihma.upv.es (J. Jaime Gómez-Hernández)
} 
MADE (MAcro-Dispersion Experiment). The present analysis focuses on the second experiment, which was performed between June 1990 and September 1991 using tritium as a non-reactive tracer. The aim of the experiment was to develop an extensive field database for validating the type of geochemical models used to predict the transport and fate of groundwater contaminants (Boggs et al., 1993). The observed tritium plume exhibits a strongly non-Fickian, highly asymmetric spreading (at the formation scale) with high concentrations maintained near the source injection area and extensive low concentrations downstream. Although there exists abundant literature on the modeling of the (so termed) anomalous spreading at the MADE site, only a few works related with this paper will be referred to in this introduction. These works can be classified into two groups according to the approach used for transport modeling.

In a first group, a number of authors have employed the classical advection-dispersion equation (ADE) to describe the strongly non-Fickian transport behavior (e.g., Adams and Gelhar, 1992; Eggleston and Rojstaczer, 1998; Barlebo et al., 2004; Salamon et al., 2007). Of these works, Salamon et al. (2007) showed that, with proper modeling of the fine-scale variability, it is possible to generate realizations of the hydraulic conductivity capable to reproduce the observed tracer movement, simply using the ADE. They used a holeeffect variogram model to characterize the flowmeter-derived conductivities. The final realizations displayed the apparent periodicity of the observed conductivities, which was enough to induce the type of spreading observed in the experiment. However, in practice, it is difficult to work with this type of high-resolution models, involving millions of nodes, particularly if multiple realizations are to be analyzed. This difficulty is what motivates our paper.

In a second group, researchers have used models that go beyond the advection-dispersion model (e.g., Berkowitz and Scher, 1998; Feehley et al., 2000; Harvey and Gorelick, 2000; Benson et al., 2001; Baeumer et al., 2001; Schumer et al., 2003; Guan et al., 2008; Liu et al., 2008; Llopis-Albert and Capilla, 2009). These authors use dual-domain mass transfer models, continuous time random walk or other alternative models capable of accounting for the strongly delayed solute transport as an alternative to the classical ADE. However, these approaches are able to provide a good match to the observed field data only a posteriori; that is, they need to calibrate their model parameters once the concentration data are collected, and then, they can reproduce, almost perfectly, any departure from Fickian transport. These works prove that there are alternative transport models able to explain the MADE data; however, at this point, they lack predictive capabilities since their parameters can only be determined after the experiment is done.

All of these studies had varying degrees of success in reproducing the spreading of the tracer plume. For instance, Barlebo et al. (2004) obtained a good reproduction of the irregular plume using the ADE after 
calibrating the concentration measurements and head data. However, calibrated hydraulic conductivities resulted a factor of five larger than the flowmeter-derived measurements. The authors attributed this discrepancy to a systematical measurement error. The accuracy of the flowmeter-derived conductivities and of the measured concentrations have raised further discussions (see Molz et al., 2006; Hill et al., 2006).

Our work builds on the study by Salamon et al. (2007) with the purpose to show that the observed transport spreading at the MADE site can also be reproduced on a coarse model by the ADE. A highresolution hydraulic conductivity realization is selected from the study by Salamon et al. (2007) and it is upscaled onto a coarser model with several orders of magnitude less elements. This upscaling approach, if successful, would permit multiple realization analyses since it would reduce significantly the computational effort needed to obtain the solute evolution at the site. Unlike previous studies of upscaling focusing on two-dimensional examples or synthetic experiments (e.g., Warren and Price, 1961; Gómez-Hernández, 1991; Durlofsky et al., 1997; Chen et al., 2003), we analyze, with real data, a variety of three-dimensional (3D) hydraulic conductivity upscaling techniques ranging from simple averaging over a uniform grid to sophisticated Laplacian-based upscaling approaches on non-uniform grids. To the best of our knowledge, this is the first time that an analysis of this type has been performed in a real 3D case. Since we will be testing the use of a full tensor representation of conductivities in the upscaled model, our group had to develop a computer code (Li et al., 2010), which has been placed on the public domain, specifically designed to solve the finite-difference approximation of the groundwater flow equation without assuming that the principal directions of the hydraulic conductivity tensors are aligned to the reference axes.

The remaining of this paper is organized as follows. First, in section 2, we summarize the findings by Salamon et al. (2007) who used a hole-effect variogram model to describe the spatial variability of $\ln K$ and, thus, were able to reproduce the non-Fickian solute spreading observed in the field. Out of the several realizations analyzed by Salamon et al. (2007), we select the one with the best reproduction of the solute spreading. This realization will be used as the reference to test different upscaling approaches. Second, in section 3, simple average, simple-Laplacian, Laplacian-with-skin and non-uniform coarsening upscaling methods are revisited from the perspective of their numerical implementation. Third, in section 4 , the flow and transport numerical models are discussed, and the benefits/limitations of using different upscaling methods at the MADE site are quantified and evaluated. Next, in section 5, there is a general discussion. Finally, in section 6, we summarize the main results and conclusions of this paper. 


\section{Modeling transport at the MADE site}

In this work, we focus on the tritium data collected in the second MADE experiment. An extensive discussion of the main geological features and hydrogeological characterization of the site has been given by Boggs et al. (1992), Adams and Gelhar (1992), Rehfeldt et al. (1992), and Boggs and Adams (1992). Salamon et al. (2007) found that the non-Fickian solute spreading observed in the field could be reproduced using the standard advection-dispersion model as long as the spatial variability of hydraulic conductivity is properly characterized at the fine scale. For the sake of completeness, next we briefly comment the results by Salamon et al. (2007).

The geostatistical analysis of the 2495 flowmeter-derived hydraulic conductivity measurements obtained at 62 boreholes (see Figure 1) indicates that the spatial variability of $\ln K$ shows a pseudo-periodic behavior in the direction of flow (Figure 2). This behavior is modeled using a hole-effect variogram, which is nested with a nugget effect and a spherical variogram as given by:

$$
\gamma(\mathbf{h})=c_{0}+c_{1} \cdot \operatorname{Sph}\left(\left\|\frac{h_{x}}{a_{x_{1}}}, \frac{h_{y}}{a_{y_{1}}}, \frac{h_{z}}{a_{z_{1}}}\right\|\right)+c_{2} \cdot\left[1-\cos \left(\left\|\frac{h_{x}}{a_{x_{2}}}, \frac{h_{y}}{a_{y_{2}}}, \frac{h_{z}}{a_{z_{2}}}\right\| \pi\right)\right]
$$

where $\mathbf{h}=\left(h_{x}, h_{y}, h_{z}\right)$ is the separation vector, $a_{x_{1}}, a_{y_{1}}, a_{z_{1}}$ are the ranges of the spherical variogram, $a_{x_{2}}, a_{y_{2}}, a_{z_{2}}$ are the ranges of the hole-effect variogram, $\|\cdot\|$ denotes vector modulus, $c_{0}$ is the nugget, $c_{1}$ is the sill of the spherical model, $c_{2}$ is the sill of the hole-effect model, with the $y$-axis oriented parallel to the flow direction, the $x$-axis is orthogonal to it on the horizontal plane, and the $z$-axis is parallel to the vertical direction. The parameter values used to fit the experimental variogram are given in Table 1. Notice that $a_{y_{2}}$, and $a_{z_{2}}$ are equal to infinity, meaning that the hole-effect is only present along the flow direction. The fitted model is also shown in Figure 2.

The computational domain is a parallelepiped with dimensions of $x=110 \mathrm{~m}, y=280 \mathrm{~m}, z=10.5 \mathrm{~m}$ and it is discretized in 2156000 cells of size $\Delta x=\Delta y=1.0 \mathrm{~m}$, and $\Delta z=0.15 \mathrm{~m}$ (see Figure 1). Cell size, according to Salamon et al. (2007), is similar in magnitude with the support scale of the flowmeter measurements. The aquifer is modeled as confined with impermeable boundaries on the faces parallel to flow, and constant head boundaries on the faces orthogonal to it. The values prescribed at the constant head boundaries are obtained by kriging the head averages over one-year observed in the nearby piezometers.

Salamon et al. (2007) used the random walk particle tracking code RW3D (Fernàndez-Garcia et al., 2005) to simulate solute transport. The local-scale longitudinal dispersivity was set as $0.1 \mathrm{~m}$, which corresponds approximately to the value calculated by Harvey and Gorelick (2000). Transverse horizontal and vertical 
local-scale dispersivity values were chosen to be one order of magnitude smaller than the longitudinal dispersivity, i.e., $0.01 \mathrm{~m}$. Apparent diffusion for tritium was set to $1.0 \mathrm{~cm}^{2} / \mathrm{d}$ (Gillham et al., 1984). An average total porosity of 0.32 as determined from the soil cores by Boggs et al. (1992) was assigned uniformly to the entire model area. The observed mass distribution on the $27^{\text {th }}$ day was employed to establish the initial concentration distribution. A simple interpolation of the initial concentrations was used to establish the concentrations in the model cells, and then 50000 particles were distributed accordingly. The observed mass distribution on the $328^{\text {th }}$ day was used to obtain reference mass profile distributions to which the model is compared. These longitudinal profiles were obtained by integrating the mass from 28 equally-spaced vertical slices, each of $10 \mathrm{~m}$ width and parallel to flow. All results are displayed after normalizing the mass by the total mass injected. Figure 3 shows the longitudinal mass distribution profiles obtained by Salamon et al. (2007) after transport simulation on 40 realizations generated by sequential Gaussian simulation. These realizations were generated using the code GCOSIM3D, (Gómez-Hernández and Journel, 1993) with the variogram model given by equation (1) and the parameter values from Table 1 . Out of these 40 realizations, solute transport on realization number 26 shows a spatial spread similar to the one observed in the field. For this reason, this conductivity realization is chosen as the reference field to test the different upscaling methods. Figure 4 shows the hydraulic conductivity field of realization number 26 .

Up to here, we have limited ourselves to briefly describe the specific results from Salamon et al. (2007) that this work uses as starting point. We are not trying to re-analyze MADE, but rather to demonstrate that careful hydraulic conductivity upscaling can be used to model flow and transport in highly heterogeneous fields exhibiting, at the formation scale, a non-Fickian behavior. To evaluate the upscaling procedure we will compare flow and transport in realization \#26 before and after upscaling, aiming at obtaining the same results. Obviously, the departure of transport results computed on realization \#26 from the experimental data will remain after upscaling. Trying to get the best reproduction of the experimental data will require a further calibration exercise that is not the objective of this paper.

\section{Hydraulic conductivity upscaling}

Although hydraulic conductivity upscaling has been disregarded by some researchers on the basis that the increase of computer capabilities will make it unnecessary, there will always be a discrepancy between the scale at which we can characterize the medium, and the scale at which we can run the numerical codes. This discrepancy makes upscaling necessary to transfer the information collected at the measurement scale into a coarser scale suitable for numerical modeling. The need for upscaling is even more justified when performing 
uncertainty analysis in a Monte Carlo framework requiring the evaluation of multiple realizations. Excellent reviews on upscaling geology and hydraulic conductivity are given by Wen and Gómez-Hernández (1996b), Renard and Marsily (1997) and Sánchez-Vila et al. (2006). In this section, we briefly revisit the most commonly used upscaling techniques with an emphasis on their numerical implementation procedures.

\subsection{Simple averaging}

It is well known that, for one-dimensional flow in a heterogeneous aquifer, the equivalent hydraulic conductivity $\left(K^{b}\right)$ that, for a given hydraulic head gradient, preserves the flows crossing the aquifer is given by the harmonic mean of the hydraulic conductivities (Freeze and Cherry, 1979). In two-dimensional flow for media with isotropic spatial correlation and a lognormal probability distribution, the geometric mean provides good block conductivities (Matheron, 1967); Gómez-Hernández and Wen (1994) and Sánchez-Vila et al. (1996) used synthetic experiments to corroborate this conclusion.

Some heuristic rules have been proposed for three-dimensional upscaling. Cardwell and Parsons (1945) had already shown that the block conductivity should lie between the arithmetic mean and the harmonic mean when Journel et al. (1986) proposed the use of power averages (also referred to as $\omega$-norms) to estimate block conductivities. The power average is given by:

$$
K^{b}=\left\{\frac{1}{V(\mathbf{x})} \int_{V(\mathbf{x})}\left(K_{x}\right)^{\omega} d V\right\}^{1 / \omega}
$$

where $V(\mathbf{x})$ indicates the volume of the block; $K^{b}$ is the block conductivity, and $K_{x}$ represents the cell conductivities within the block, the power $\omega$ may vary from -1 , yielding the harmonic mean, to +1 , yielding the arithmetic mean, with $\omega=0$ corresponding to the geometric mean. Although Desbarats (1992) demonstrated that $\omega$ equals $1 / 3$ in $3 \mathrm{D}$ for statistically isotropic and mildly heterogeneous formations, the power coefficient $(\omega)$ has to be obtained by resorting to numerical flow experiments in arbitrary flow fields. The main advantages of this method are its mathematic conciseness and the easiness of implementation. However, there are several limitations to this power-average approach: first, the exponent $\omega$ is site-specific and cannot be predicted in a general anisotropic heterogeneous medium except after numerical calibration experiments; second, the shape and size of the blocks are not considered.

\subsection{Simple-Laplacian}

This approach is based on the local solution, for each block being upscaled, of a variant of the Laplace equation (steady-state, groundwater flow with neither sources nor sinks). In this approach, the block con- 
ductivity is assumed to be a tensor with principal directions parallel to the coordinate axes; and therefore, diagonal for this reference system.

To determine each component of the tensor, a local problem is solved inducing flow in the component direction. For instance, in $2 \mathrm{D}$, the tensor will have two components, $K_{x x}^{b}$, and $K_{y y}^{b}$; to determine the component corresponding to the $x$ direction, $K_{x x}^{b}$, the procedure would be as follows: (1) extract the block being upscaled and solve the groundwater flow equation just within the block, at the fine scale with no flow boundaries on the sides parallel to flow and prescribed heads on the sides perpendicular to flow as shown in Figure 5; (2) evaluate the total flow $Q$ through any cross-section parallel to the $y$-axis from the solution of the flow equation, and (3) compute the block conductivity tensor component in the $x$-direction as:

$$
K_{x x}^{b}=-\left(\frac{Q}{y_{1}-y_{0}}\right) /\left(\frac{h_{1}-h_{0}}{x_{1}-x_{0}}\right)
$$

where $y_{1}-y_{0}$ is the block width; $h_{1}-h_{0}$ is the difference between the prescribed heads on the opposite sides of the block (see Figure 5), and $x_{1}-x_{0}$ is the block length. $K_{y y}^{b}$ would be obtained similarly after solving a similar local flow problem with the boundary conditions in Figure 5 rotated $90^{\circ}$.

The main shortcoming of this approach is that the assumption of a diagonal tensor is not well-founded for a heterogeneous aquifer. In other words, the heterogeneity within the block may induce an overall flux that is not parallel to the macroscopic head gradient, a behavior that cannot be captured with a diagonal tensor

This method has been widely used to calculate block conductivities in petroleum engineering and hydrogeology (e.g., Warren and Price, 1961; Bouwer, 1969; Journel et al., 1986; Desbarats, 1987, 1988; Deutsch, 1989; Begg et al., 1989; Bachu and Cuthiell, 1990). More recently Sánchez-Vila et al. (1996) utilized this approach to study the scale effects in transmissivity; Jourde et al. (2002) used it to calculate block equivalent conductivities for fault zones; and Flodin et al. (2004) used this method to illustrate the impact of boundary conditions on upscaling. It has also been employed by Fernàndez-Garcia and Gómez-Hernández (2007) and Fernàndez-Garcia et al. (2009) to evaluate the impact of hydraulic conductivity upscaling on solute transport. Some reasons favoring this approach are that it is not empirical but phenomenological, i.e., it is based on the solution of the groundwater flow equation, and it yields a tensor representation of the block conductivity, which would be exact for the case of perfectly layered media, with the layers parallel to the coordinate axes. 


\subsection{Laplacian-with-skin}

To overcome the shortcomings of the simple-Laplacian approach, the Laplacian-with-skin approach was presented by Gómez-Hernández (1991). In this approach, the block conductivity is represented by a generic tensor (not necessarily diagonal) and the local flow problem is solved over an area that includes the block plus a skin surrounding it (see Figure 6). The skin is designed to reduce the impact of the arbitrary boundary conditions used in the solution of the local flow problems letting the conductivity values surrounding the block to take some control on the flow patterns within the block.

For a 3D block, the overall algorithm is summarized as follows: (1) the block to upscale plus the skin is extracted from the domain; (2) flow is solved at the fine scale within the block-plus-skin region for a series of boundary conditions; (3) for each boundary condition the spatially-averaged specific discharge (q) and gradient $(\mathbf{J})$ are calculated as,

$$
\left\langle q_{i}\right\rangle=\frac{1}{V(\mathbf{x})} \int_{V(\mathbf{x})} q_{i}(\mathbf{x}) d \mathbf{x}
$$

$$
\left\langle J_{i}\right\rangle=\frac{1}{V(\mathbf{x})} \int_{V(\mathbf{x})} \frac{\partial h(\mathbf{x})}{\partial x_{i}} d \mathbf{x}
$$

where $i$ refers to the three components of the vectors (i.e., $q_{x}, q_{y}$ and $q_{z} ; J_{x}, J_{y}$ and $J_{z}$ ); and (4) the tensor components of $\mathbf{K}^{b}$ are determined by solving the following overdetermined system of linear equations by a standard least squares procedure (Press et al., 1988).

$$
\left[\begin{array}{cccccc}
\left\langle J_{x}\right\rangle_{1} & \left\langle J_{y}\right\rangle_{1} & \left\langle J_{z}\right\rangle_{1} & 0 & 0 & 0 \\
0 & \left\langle J_{x}\right\rangle_{1} & 0 & \left\langle J_{y}\right\rangle_{1} & \left\langle J_{z}\right\rangle_{1} & 0 \\
0 & 0 & \left\langle J_{x}\right\rangle_{1} & 0 & \left\langle J_{y}\right\rangle_{1} & \left\langle J_{z}\right\rangle_{1} \\
\left\langle J_{x}\right\rangle_{2} & \left\langle J_{y}\right\rangle_{2} & \left\langle J_{z}\right\rangle_{2} & 0 & 0 & 0 \\
0 & \left\langle J_{x}\right\rangle_{2} & 0 & \left\langle J_{y}\right\rangle_{2} & \left\langle J_{z}\right\rangle_{2} & 0 \\
0 & 0 & \left\langle J_{x}\right\rangle_{2} & 0 & \left\langle J_{y}\right\rangle_{2} & \left\langle J_{z}\right\rangle_{2} \\
\cdots & \cdots & \ldots & \cdots & \cdots & \cdots \\
\left\langle J_{x}\right\rangle_{n} & \left\langle J_{y}\right\rangle_{n} & \left\langle J_{z}\right\rangle_{n} & 0 & 0 & 0 \\
0 & \left\langle J_{x}\right\rangle_{n} & 0 & \left\langle J_{y}\right\rangle_{n} & \left\langle J_{z}\right\rangle_{n} & 0 \\
0 & 0 & \left\langle J_{x}\right\rangle_{n} & 0 & \left\langle J_{y}\right\rangle_{n} & \left\langle J_{z}\right\rangle_{n}
\end{array}\right] \cdot\left[\begin{array}{c}
K_{x x}^{b} \\
K_{x y}^{b} \\
K_{x z}^{b} \\
K_{y y}^{b} \\
K_{y z}^{b} \\
K_{z z}^{b}
\end{array}\right]=-\left[\begin{array}{c}
\left\langle q_{x}\right\rangle_{1} \\
\left\langle q_{y}\right\rangle_{1} \\
\left\langle q_{z}\right\rangle_{1} \\
\left\langle q_{x}\right\rangle_{2} \\
\left\langle q_{y}\right\rangle_{2} \\
\left\langle q_{z}\right\rangle_{2} \\
\cdots \\
\left\langle q_{x}\right\rangle_{n} \\
\left\langle q_{y}\right\rangle_{n} \\
\left\langle q_{z}\right\rangle_{n}
\end{array}\right]
$$

where $1, \ldots, n$ refers to the different boundary conditions; $K_{x x}^{b} \cdots K_{z z}^{b}$ are the components of the upscaled equivalent conductivity tensor $\mathbf{K}^{b}$. In principle, in $3 \mathrm{D}$, two sets of boundary conditions are sufficient to 
determine $\mathbf{K}^{b}$. However, from a practical point of view, the number of boundary conditions should be greater than two $(n>2)$ to better approximate all possible flow scenarios.

Every three rows in Equation (6) are the result of enforcing Darcy's law on the average values in equations (4) and (5) for a given boundary condition:

$$
\langle\mathbf{q}\rangle=-\mathbf{K}^{b}\langle\mathbf{J}\rangle
$$

The block conductivity tensor must be symmetric and positive definite. Symmetry is easily enforced by making $K_{x y}^{b}=K_{y x}^{b}, K_{x z}^{b}=K_{z x}^{b}$ and $K_{y z}^{b}=K_{z y}^{b}$. Positive definiteness is checked a posteriori. In case the resulting tensor is non-positive definite, the calculation is repeated either with more boundary conditions or with a larger skin size (Wen et al., 2003; Li et al., 2011).

We note that the critical point in this approach is the selection of the set of $n$ alternative boundary conditions. In general, this set of boundary conditions is chosen so as to induce flow in several directions (for instance, the prescribed head boundary conditions in Figure 6 induce flow at $0^{\circ}, 45^{\circ}, 90^{\circ}$ and $135^{\circ}$ angles with respect to the $x$-direction). For the boundary conditions, we have chosen to prescribe linearly varying heads along the sides of the blocks, other authors (Durlofsky, 1991) have proposed the use of periodic boundary conditions. Flodin et al. (2004) showed that the resulting block conductivities do not depend significantly on whether the boundary conditions are linearly varying or periodic.

\subsection{Non-uniform coarsening}

Prior to upscaling, the fine-scale realization has to be overlain with the coarse-scale discretization that will be used in the numerical model. Each block in the coarse discretization must be assigned an upscaled conductivity value on the basis of the conductivity values in the fine-scale realization. Initially, all studies on hydraulic conductivity upscaling assumed that the coarse scale discretization was uniform, that is, all coarse blocks were of the same shape and size, until Durlofsky et al. (1997) introduced the concept of nonuniform coarsening. The rationale was simple, if upscaling induces smoothing, and the petroleum engineer is most interested in the water cut (the early breakthrough at the production wells when petroleum is being displaced by injected water) it is important to smooth the least the areas of high displacement velocities, whereas the smoothing in the areas of low velocities is less relevant. For this purpose, Durlofsky et al. (1997) suggest the following steps: (1) identify the underlying high velocity regions using a fine-scale single-phase flow simulation; (2) on the basis of this simulation define a discretization with small blocks in high-velocity 
areas and large ones elsewhere; and (3) apply the Laplacian-with-skin upscaling technique to calculate the block conductivity tensors of the coarse (non-uniform) blocks.

In a hydrogeological context, we can also use a non-uniform coarsening aimed to preserve small blocks in: (1) high flow velocity zones; (2) regions where hydraulic gradients change substantially over short distances, such as near pumping or injection wells (Wen and Gómez-Hernández, 1998); (3) areas near contaminant spills within a regional aquifer where accurate simulation of plume movement is of interest; and (4) in zones requiring a detailed representation of heterogeneity, for instance to capture channels or fractures (Durlofsky et al., 1997; Wen et al., 2003; Flodin et al., 2004).

\section{Coarse model and simulation results}

In this section, we first present the governing equation and the solution procedures for the flow and transport models, and then we discuss the results obtained applying the different upscaling techniques described in the previous section. All of these techniques are applied to realization \#26 of the MADE aquifer in Salamon et al. (2007).

\subsection{Coarse Flow and Transport Equations}

Under steady-state flow conditions and in the absence of sinks and sources, the flow equation of an incompressible or slightly compressible fluid in saturated porous media can be expressed by combining Darcy's Law and the continuity equation, which in Cartesian coordinates is (Bear, 1972; Freeze and Cherry, 1979):

$$
\nabla \cdot(\mathbf{K}(\mathbf{x}) \nabla h(\mathbf{x}))=0
$$

where $h$ is the piezometric head, and $\mathbf{K}$ is a second-order symmetric hydraulic conductivity tensor.

Most frequently, the hydraulic conductivity tensor is assumed isotropic and therefore can be represented by a scalar. In this case, a standard seven-point block-centered finite-difference stencil is typically employed to solve the partial differential equation in three dimensions. This approach is also valid if, for all blocks, the conductivity is modeled as a tensor with the principal directions aligned with the block sides (Harbaugh et al., 2000). However, when modeling geologically complex environments at a coarse scale, the assumption of isotropic block conductivity or even tensor conductivity with principal components parallel to the block sides is not warranted. It is more appropriate to use a full hydraulic conductivity tensor to capture properly the average flow patterns within the blocks (Bourgeat, 1984; Gómez-Hernández, 1991; Wen et al., 2003; Zhou 
et al., 2010). Recently, the commonly used groundwater model software MODFLOW implemented a new module that allows the use of a full tensorial representation for hydraulic conductivity within model layers (Anderman et al., 2002) which has been successfully applied in 2D examples such as in Fernàndez-Garcia and Gómez-Hernández (2007).

Modeling three-dimensional flow in a highly heterogeneous environment at a coarse scale, requires accounting for a tensorial representation of hydraulic conductivity. We cannot assume, a priori that specific discharge and hydraulic head gradient will be parallel, nor that the principal directions of the hydraulic conductivity tensors are the same in all blocks. For this reason, and given that MODFLOW can only account for 3D tensors if one of its principal directions is aligned with the vertical direction, Li et al. (2010) developed a three-dimensional groundwater flow simulation with tensor conductivities of arbitrary orientation of their principal directions. This code is based on an nineteen-point finite-difference approximation of the groundwater flow equation, so that the flow crossing any block interface will depend not only on the head gradient orthogonal to the face, but also on the head gradient parallel to it.

Finite-difference modeling approximates the specific discharges across the interface between any two blocks $i$ and $j$ as a function of the hydraulic conductivity tensor in between block centers. This tensor is neither the one of block $i$ nor of the one of block $j$. For this reason, finite-difference numerical models need to approximate the interblock conductivity; the most commonly used approximation is taking the harmonic mean of adjacent block values. When block conductivities are represented by a tensor, the concept of how to average the block tensors in adjacent blocks is not clear. To overcome this difficulty, the code developed by Li et al. (2010) takes directly, as input, interblock conductivity tensors, removing the need of any internal averaging of tensors defined at block centers. Within the context of upscaling, deriving the interblock conductivity tensors simply amounts to isolate the parallelepiped centered at the interface between adjacent blocks, instead of isolating the block itself, and then apply the upscaling techniques described in the previous section. In other contexts, the user must supply the interblock conductivity tensors directly. Several authors (Appel, 1976; Gómez-Hernández, 1991; Romeu and Noetinger, 1995; Li et al., 2010) have recommended to work directly with interblock conductivities for more accurate groundwater flow simulations.

The details of the algorithm used to solve the flow equation are provided in Li et al. (2010) and summarized in Appendix A.

Mass transport is simulated using the advection-dispersion equation: (Bear, 1972; Freeze and Cherry, 1979): 


$$
\phi \frac{\partial C(\mathbf{x}, t)}{\partial t}=-\nabla \cdot(\mathbf{q}(\mathbf{x}) C(\mathbf{x}, t))+\nabla \cdot(\phi \mathbf{D} \nabla C(\mathbf{x}, t))
$$

where $C$ is the dissolved concentration of solute in the liquid phase; $\phi$ is the porosity; $\mathbf{D}$ is the local hydrodynamic dispersion coefficient tensor, and $\mathbf{q}$ is the Darcy velocity given by $\mathbf{q}(\mathbf{x})=-\mathbf{K}(\mathbf{x}) \nabla h(x)$.

As in the works of Salamon et al. (2007) and Llopis-Albert and Capilla (2009) at the MADE site, the random walk particle tracking code RW3D (Fernàndez-Garcia et al., 2005; Salamon et al., 2006) is used to solve the transport equation (9). In this approach, the displacement of each particle in a time step includes a deterministic component, which depends only on the local velocity field, and a Brownian motion component responsible for dispersion. A hybrid scheme is utilized for the velocity interpolation which provides local as well as global divergence-free velocity fields within the solution domain. Meanwhile, a continuous dispersion-tensor field provides a good mass balance at grid interfaces of adjacent cells with contrasting hydraulic conductivities (LaBolle et al., 1996; Salamon et al., 2006). Furthermore, in contrast to the constant time scheme, a constant displacement scheme (Wen and Gómez-Hernández, 1996a), which modifies automatically the time step size for each particle according to the local velocity, is employed in order to reduce computational effort.

\subsection{Upscaling design and error measure}

In this work, we have performed both uniform and non-uniform upscaling. In the case of uniform upscaling, the original hydraulic conductivity realization discretized into $110 \times 280 \times 70$ cells of $1 \mathrm{~m}$ by $1 \mathrm{~m}$ by $0.15 \mathrm{~m}$ is upscaled onto a model with $11 \times 28 \times 14$ blocks of $10 \mathrm{~m}$ by $10 \mathrm{~m}$ by $0.75 \mathrm{~m}$. This upscaling represents going from 2156000 cells down to 4312 blocks, i.e., a reduction by a factor of 500 . The reduction in model size, undoubtedly, reduces the computational cost for flow and transport modeling. As will be shown, the flow and transport results can be improved using a non-uniform discretization of the coarse model. For the non-uniform upscaling, the discretization continues to be a rectangular grid, with the following coarse block dimensions: along the $x$-axis (orthogonal to flow), block dimension is $10 \mathrm{~m}$, except between $x=40 \mathrm{~m}$ and $x=90 \mathrm{~m}$ where it is $5 \mathrm{~m}$; along the $y$-axis (parallel to flow), block dimension is 10 $\mathrm{m}$, except between $y=20 \mathrm{~m}$ and $y=130 \mathrm{~m}$ where it is $5 \mathrm{~m}$; and along the $z$-axis, block dimension is $1.5 \mathrm{~m}$ between $z=0 \mathrm{~m}$ and $z=3 \mathrm{~m}$ and $0.75 \mathrm{~m}$ elsewhere. The final model has $16 \times 39 \times 12(7488)$ blocks, with smaller blocks close to the source and along the area through which it is most likely that the solute plume will travel. The reduction factor in size, with respect to the initial discretization is close to 300 .

The first set of upscaling runs use simple averaging rules to obtain the block conductivity values. The 
second set of runs use the Laplacian-based approaches. Within this second set of runs we carry out a first comparison using tensor conductivity values computed at block centers versus tensor conductivities computed at the interfaces; the former requires a further averaging of adjacent block values to approximate the interblock conductivities needed by the numerical solver, whereas the latter does not. Then, after showing that interface-centered conductivity upscaling is more appropriate, the following upscaling runs are always performed with interblock conductivities.

In the application of any of the Laplacian approaches for upscaling, the local flow model that must be run for each block was solved by finite differences using the preconditioned conjugate gradient method implemented in MODFLOW (Hill, 1990) since we found it to be the fastest algorithm for the same convergence criteria.

In the Laplacian-with-skin approach, the size of the skin was taken equal to half the block size in each direction. A prior sensitivity analysis revealed that this skin size was enough to capture accurately the average flow crossing each of the upscaled blocks. Zhou et al. (2010) also found that half the block size is a good choice for the skin size in most situations. The overdetermined system of equations from which the components of the block tensor are described is built after solving nine local flow problems. In each of the local problems the prescribed heads applied to the boundaries of the block vary linearly as a function of $x, y$ and $z$ so that they impose overall head gradients parallel to the directions given by the following nine vectors $(1,0,0),(0,1,0),(0,0,1),(1,1,0),(1,0,1),(0,1,1),(1,1,0),(-1,0,1),(0,-1,1)$.

To evaluate the performance of the different upscaling techniques we focus on the reproduction of the interblock fluxes and on the reproduction of the solute transport. For the fluxes, we compare the interblock specific discharges obtained after solving the flow equation at the coarse scale with the corresponding values derived after solving the flow equation in the reference field at the fine scale. We focus on fluxes instead of piezometric heads because fluxes have a larger spatial variability and have a dominant role in solute transport. The metric we use to evaluate each technique is the average relative bias $(R B)$ given by:

$$
R B=\frac{1}{N} \sum_{i=1}^{N}\left|\frac{q_{f, i}-q_{c, i}}{q_{f, i}}\right| \cdot 100
$$

where $N$ is the number of block interfaces; $q_{f, i}$ is the specific discharge through the block interface $i$ computed from the fine scale solution, and $q_{c, i}$ is the specific discharge through the block interface $i$ resulting from the coarse scale simulation. 
Mass transport reproduction is evaluated qualitatively by comparing the longitudinal mass distribution profiles at the $328^{\text {th }}$ day obtained from the fine scale model with the one obtained from the coarse scale model.

Notice that the same transport parameters used for the fine scale simulation described in section 2 are also used for the coarse scale simulation.

\subsection{Results and Comparisons}

Next, we will discuss the flow and transport performance of the different upscaling approaches. The flow upscaling analysis excludes the interfaces of the blocks which are adjacent to the boundaries; the reason for the exclusion is that the boundary conditions have an impact on the results of upscaling in the nearby blocks (Vermeulen et al., 2006). Excluding these blocks, the discrepancies in flow reproduction between the coarse and fine scale simulations will be due to the upscaling method and not to the presence of the boundaries. This consideration is not necessary when analyzing the transport upscaling since the plume travels far enough from the boundaries. Also, since, for transport purposes, the flows along the $y$-axis are the most relevant (and of the highest magnitude), the graphs only shows the specific discharges across the interfaces orthogonal to the $y$-axis, similar results are obtained when analyzing the interfaces orthogonal to the $x$ - and $z$-axis.

Figure 7 shows the scatterplots of reference versus upscaled fluxes through the block interfaces using simple averaging methods. All circles within the dotted lines have a relative bias smaller than $10 \%$ of the reference values, whereas the circles within the solid lines have a relative bias smaller than $40 \%$. It is clear that, out of the different averages, the power average with a power of 0.5 gives the best results. The use of the harmonic mean (Figure 7A) (power average with $\omega=-1$ ) tends to severely underestimate the reference fluxes, while the arithmetic mean (Figure 7C) (power average with $\omega=+1$ ) tends to overestimate them. The geometric mean (power average with $\omega=0$ ) does a better work but stills tends to underestimate the fluxes (Figure 7B). The best average, as already pointed out by Cardwell and Parsons (1945) should be somewhere between the harmonic and the arithmetic averages. In this specific case, we found that the smallest bias occurs when $\omega=0.5$ (Figure 7D), resulting in a relative bias, RB, of 11\%. As mentioned earlier, for isotropic, mildly heterogeneous media, Desbarats (1992) found $\omega=1 / 3$ to be the best power average for upscaling purposes. In the MADE case, the field is neither isotropic, nor mildly varying ( $\ln K$ variance is close to 5 ), thus it is not surprising that the optimal power value does not coincide with the value reported by Desbarats (1992). 
Figure 8 shows the longitudinal mass distribution profile (integrated along the direction orthogonal to flow, and normalized by the total mass) of the tritium plume using different simple averaging upscaling techniques at 328 days. The solid line represents the fine scale result. For reference, the initial conditions at 27 days are also shown by the bold dashed curve. The remaining of the curves are the upscaled results for the different averages. Both the upscaled models using the arithmetic mean and the 0.5 power average are capable of reproducing the long downstream spreading of the contaminant plume, with the power mean resulting in a better representation of the distribution close to the source. Yet, none of the methods exhibits a satisfactory accuracy.

Figure 9 shows the scatterplots of reference versus upscaled fluxes using different Laplacian approaches. Figures 9A and 9B display upscaling approaches using a simple-Laplacian (i.e., without skin, and assuming diagonal tensors) for block-centered and interblock-centered upscaling, respectively. It is clear that it is better to upscale directly the interblock conductivity than upscaling the block values and then let the numerical model estimate internally the interblock conductivity. This is consistent with earlier studies (Li et al., 2010).

Figures 9B and 9C display two different Laplacian approaches without skin. The simple-Laplacian in Figure 9B assumes a diagonal representation of the tensor in the reference axes, whereas the Laplacian-withskin but with a skin set to zero in Figure 9C allows for the tensor representation to be non-diagonal. Allowing the tensor principal components not to be aligned with the reference axes results in a better representation of the fluxes, since it is unlikely that all interblocks would have conductivities with principal directions parallel to the reference axes.

Moreover, if the skin is allowed to increase up to half the block size, the results improve even further, as can be checked by comparing Figures 9C and 9D. This improvement can be related to the reduction of the influence in the flow patterns within the block of the boundary conditions used in the local flow models in favor of the influence of the nearby conductivities from the reference aquifer.

Since most of the commonly available groundwater flow simulators only accept diagonal tensors as input parameter values, a test was made by solving the flow and transport in the coarse scale ignoring the offdiagonal components of the tensors used in Figure 9D. The results are shown in Figure 9E and they are qualitatively similar to those in Figure 9D. In this specific case, in which the reference axes of the numerical model are aligned with the main directions of the statistical anisotropy of hydraulic conductivity it could be expected that the off-diagonal components of the upscaled block conductivity tensors were small, and therefore, flow predictions neglecting them go almost unaffected. In a general setting with complex geology, cross-beddings, or non-uniform anisotropies, the use of a full tensor block conductivity would be necessary 
for a good reproduction of the aquifer response (Bierkens and Weerts, 1994).

Finally, Figure $9 \mathrm{~F}$ shows that the best results are achieved when the upscaling is performed on a nonuniform coarse grid, which has been refined in the areas of highest velocities (see grid in Figure 15), using an interface-centered Laplacian-with-skin upscaling. While this result is expected, since the number of model blocks is larger in the non-uniform grid, the improvement is not due just to having almost twice as many blocks, but to the fact, that these many more blocks are located in the zones where the variability of velocity is the highest. The message to take away is that it is advantageous to use a non-uniform coarse grid and that the definition of this grid is very important to achieve the best upscaling results. Other authors have investigated along these lines and have proposed the use of flexible grids which maintain a given topology (basically keeping constant the number of rows, columns and layers) but which are deformed so as to reduce the variability of the specific discharge vector within each coarse block (i.e, Garcia et al., 1992; Wen and Gómez-Hernández, 1998).

Figure 10 compares the mass longitudinal profile of the upscaling approaches in Figures 9A (uniform grid, simple-Laplacian, block-centered), 9B (uniform grid, simple-Laplacian, interblock-centered) and 9D (uniform grid, Laplacian-with-skin, interblock-centered) with the reference profile at day 328 . The improvement in the reproduction of the reference values by the difference upscaling techniques shows a similar progression as the improvement seen in the reproduction of the fluxes in Figure 9. Comparing these curves to any of the curves in Figure 8, which were obtained with simple averaging upscaling rules, it is clear that any upscaling approach based on a local solution of the flow equation provides a better representation of the hydraulic conductivity distribution and yields better transport predictions. The two interblock-aimed upscaling approaches are able to capture both the peak concentration near the source and the downstream spreading.

Figure 11 shows the mass longitudinal profile of the upscaling approaches in Figures 9D (uniform grid, Laplacian-with-skin, interblock-centered) and 9F (non-uniform grid, Laplacian-with-skin, interblockcentered). It is evident that the non-uniform coarsening gives again the best results: up to a downstream distance of $200 \mathrm{~m}$, the reproduction is almost perfect, and the very low concentrations for distances farther than $200 \mathrm{~m}$ are adequately reproduced.

A final comparison of the different approaches can be performed by analyzing the spatial distribution of the contaminant plume, both in plan view (depth integrated) and lateral view (integrated along the $x$ axis). Figure 12 shows the contaminant plume in the reference fine-scale conductivity realization. Figures 13,14 , and 15 show the corresponding distributions for the mass transport simulation in the upscaled fields using a block-centered, simple-Laplacian upscaling approach, an interblock-centered, Laplacian-with-skin 
approach, and the non-uniform coarsening, interblock-centered, Laplacian-with-skin approach, respectively. It is evident that the block-centered approach is not capable to produce a field in which the solute travels as far downstream as in the reference field, while the most elaborated upscaling approach of Figure 15 gives results which quite closely resemble the reference values.

\section{Discussion}

We have shown that flow and transport can be modeled at the MADE site by the advection dispersion equation on relatively coarse discretization if the spatial variability of hydraulic conductivity at the fine scale is properly characterized and a careful upscaling approach is applied to it. But, why is this so? and why is the non-uniform grid interblock-centered Laplacian-with-skin upscaling the approach to use?

Let's first analyze the progression in the reproduction of the specific discharges with the upscaling approaches. It is well known that the coarse-scale representation of conductivity as a tensor is mostly due to the statistical anisotropy at the fine scale (Lake, 1988). In the limit, with infinite correlation in the horizontal plane, the medium would be perfectly layered and the tensor conductivity will have arithmetic average for the horizontal components and the harmonic average for the vertical ones. At the MADE site, the horizontal continuity is not infinity, but it is quite large compared with the size of the domain, this is the reason why, for the reproduction of the specific discharges across the interfaces which are orthogonal to the direction of maximum continuity, the best average is a power-average with exponent in between those corresponding to the geometric and arithmetic averages, and larger than the theoretical value for statistically isotropic media. Yet, assuming that the conductivity is a scalar (as is done when a simple average is used) implies that it is isotropic to flow. At the MADE site there is still enough anisotropic heterogeneity within the blocks to warrant the need of a tensor to describe hydraulic conductivity at the coarse scale. This is why all the Laplacian-based approaches perform better than the simple averaging ones.

Of the Laplacian-based approaches, it is shown that computing tensor conductivities at block centers and then taking the harmonic average of the components corresponding to the directions orthogonal to adjacent interfaces introduces a noise that can be eliminated by aiming directly at upscaling the interblock conductivity tensor to feed directly into the numerical simulator. This is why all interface-centered approaches outperform the block-centered approach.

Of the interblock-centered approaches, analyzing the local flow within an area extending beyond the limits of the block being upscaled (that is, including a skin) also improves the upscaling. The reason being, that the upscaled conductivities are always nonlocal (Neuman and Orr, 1993; Indelman and Abramovich, 
1994), that is, they depend not only on the fine-scale conductivities within the block, but on the ones outside, too. Extracting the block to upscale, plus a skin area surrounding it, and applying the boundary conditions of the local flow problems outside the skin, reduces the impact of the boundary conditions inside the block and allows the immediately surrounding fine scale conductivities to impose some control on the flow patterns within the block (as it will happen when the block is embedded in the aquifer).

The Laplacian-with-skin approach provides a tensor with arbitrary orientation of its principal directions. For the MADE site, it appears that assuming that the principal directions of the block hydraulic conductivity tensors are parallel to the reference axes for all blocks, does not seem to introduce too large an error (compare Figures 9D and 9E), something that could be explained on the basis that the statistical anisotropy model used has its principal directions of continuity aligned with the reference axes for the entire domain. In cases such as cross-bedded formations, or aquifers with a heterogeneity description for which anisotropy varies locally with the domain, the assumption that the principal directions are parallel to the reference axes could not be sustained.

Upscaling induces heterogeneity smoothing, by defining a non-uniform coarse grid that tries to reduce the smoothing on those areas with the highest velocities, and also on areas where fluid velocity will have the largest impact in transport predictions, the results after upscaling will be better than if we define a uniform coarse grid. Although this may appear as a trivial result, it often is disregarded.

But a good reproduction of the fluxes at the coarse scale is not guarantee that transport predictions will be equally good. It has been shown (Fernàndez-Garcia and Gómez-Hernández, 2007; Fernàndez-Garcia et al., 2009; Li et al., 2011) that, in some occasions, after coarsening a hydraulic conductivity grid, the removal of the within-block heterogeneity requires some type of transport upscaling, either modifying the transport parameters (such as enhancing dispersivity) or including transport processes besides advection and dispersion (such as mass transfer). Recall that in our work we kept the same transport equation, with the same parameter values for the fine and coarse scale simulations. But, for the MADE site this is not necessary. The reason is related on how much smearing out of the within-block heterogeneity is induced by the conductivity upscaling. When this smearing out is important, then, there is a need to include other processes; but for the MADE site and the chosen upscaling, this is not the case. The ratio between the coarse block size and the correlation ranges of the fine scale conductivities is substantially smaller than one, in the direction of flow, the ratio is $1 / 8$, in the horizontal plane orthogonal to flow, the ratio is $1 / 3.2$ and in the vertical direction is $1 / 5.5$; this means that the variability of logconductivity within the block is much smaller than the overall variance of 4.5, and therefore the heterogeneity wiped out by the upscaling process 
is not as large as to require a further transport upscaling. In the references cited above, the size of the block was on the order of magnitude of the correlation range of the underlying hydraulic conductivity if not larger, and, therefore, upscaling on those cases implied an important smoothing of heterogeneity that had to be taken into account in the transport simulation at the coarse scale.

Can the findings from this work be extrapolated to other case studies? We believe that, regarding flow upscaling, yes they can. In fact, the findings from this paper are in agreement with similar works in other case studies. However, regarding transport upscaling, they can be extrapolated only under the same conditions considered here, that is, using coarse blocks smaller than the correlation range, and, using a non-uniform grid with smaller blocks in the areas with highest velocities and in the areas through which the plume will travel.

The final point of discussion is why we have worked trying to reproduce flow and transport on a realization from Salamon et al. (2007) instead of trying to reproduce the available experimental data. This paper did not try to perform a calibration exercise of the MADE site, but rather to help in performing such a calibration in the future. With the work in this paper we show that a coarse scale model, obtained by careful upscaling of a fine scale one, can reproduce the type of transport behavior observed at the MADE site simply using the advection dispersion equation. Trying to calibrate a two-million cell model as obtained by Salamon et al. (2007) is not an easy task, it would require running many times the flow and transport models in many realizations of the site; but those runs would be possible on the coarse models used in our work. The next step in this direction would be to develop a calibration approach that would account for the upscaling step needed to reduce the numerical modeling effort. In its application of such an approach, considering heterogeneity in porosity may also help in obtaining the best calibration; something not needed in our upscaling exercise, since we assume constant porosity attached to the reference conductivity realization.

\section{Summary and Conclusions}

In this paper, we have presented a detailed analysis of the impact of different upscaling techniques on the reproduction of solute transport at the MADE site. We use as a reference a fine scale realization taken from the work by Salamon et al. (2007) that is able to reproduce the contaminant spreading observed in the experiment using an advection-dispersion model. The techniques analyzed span from simple averaging to the estimation of block tensors by local flow models. We have also analyzed the impact that non-uniform coarsening may have in the quality of the results.

This work has three main and important conclusions: 
1. In complex environments, such as the MADE site, with hydraulic conductivities which vary over many orders of magnitude, and display an intricate spatial variability, choosing an elaborated upscaling technique yields the best flow and transport results. In particular, the upscaling technique that best performs is the one that computes interblock-centered conductivity tensors using a local solution of the flow equation over a domain including the block plus a skin.

2. A non-uniform coarsening focused in the refinement of the regions through which the solute plume travels can further improve the results.

3. Modeling of flow and transport at the MADE site has been the object of debate for many years, and many complex transport models have been proposed to reproduce the plume spreading observed. We show that the advection-dispersion model can be used on a coarse model to explain the plume migration in the highly heterogeneous MADE site if careful modeling/upscaling of the flow field is performed, as long as the block size remains smaller than the correlation ranges of the underlying fine scale conductivities. 
Table 1: Variogram parameters for the model fit in Figure 2

\begin{tabular}{ccccc}
\hline Model Type & Sill & \multicolumn{3}{c}{ Range $[\mathrm{m}]$} \\
\cline { 3 - 5 } & $c$ & $a_{x}$ & $a_{y}$ & $a_{z}$ \\
\hline Nugget & 0.424 & & & \\
Spherical & 3.820 & 32 & 80 & 4.1 \\
Hole effect & 0.891 & $\infty$ & 80 & $\infty$ \\
\hline
\end{tabular}




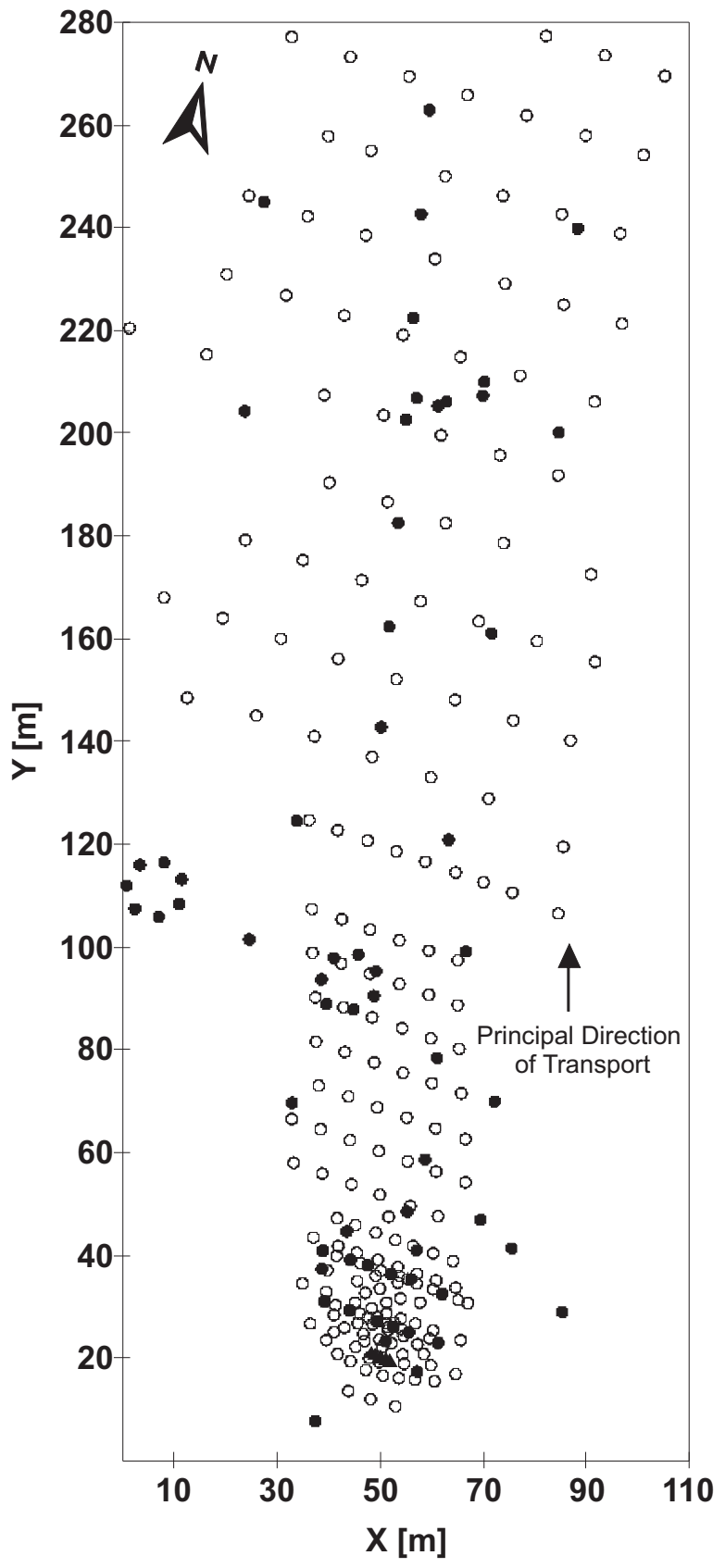

Figure 1: Plan view of model domain. Open circles denote multilevel sampler wells. Triangles indicate the tracer injection wells. Solid circles correspond to flowmeter well locations. 

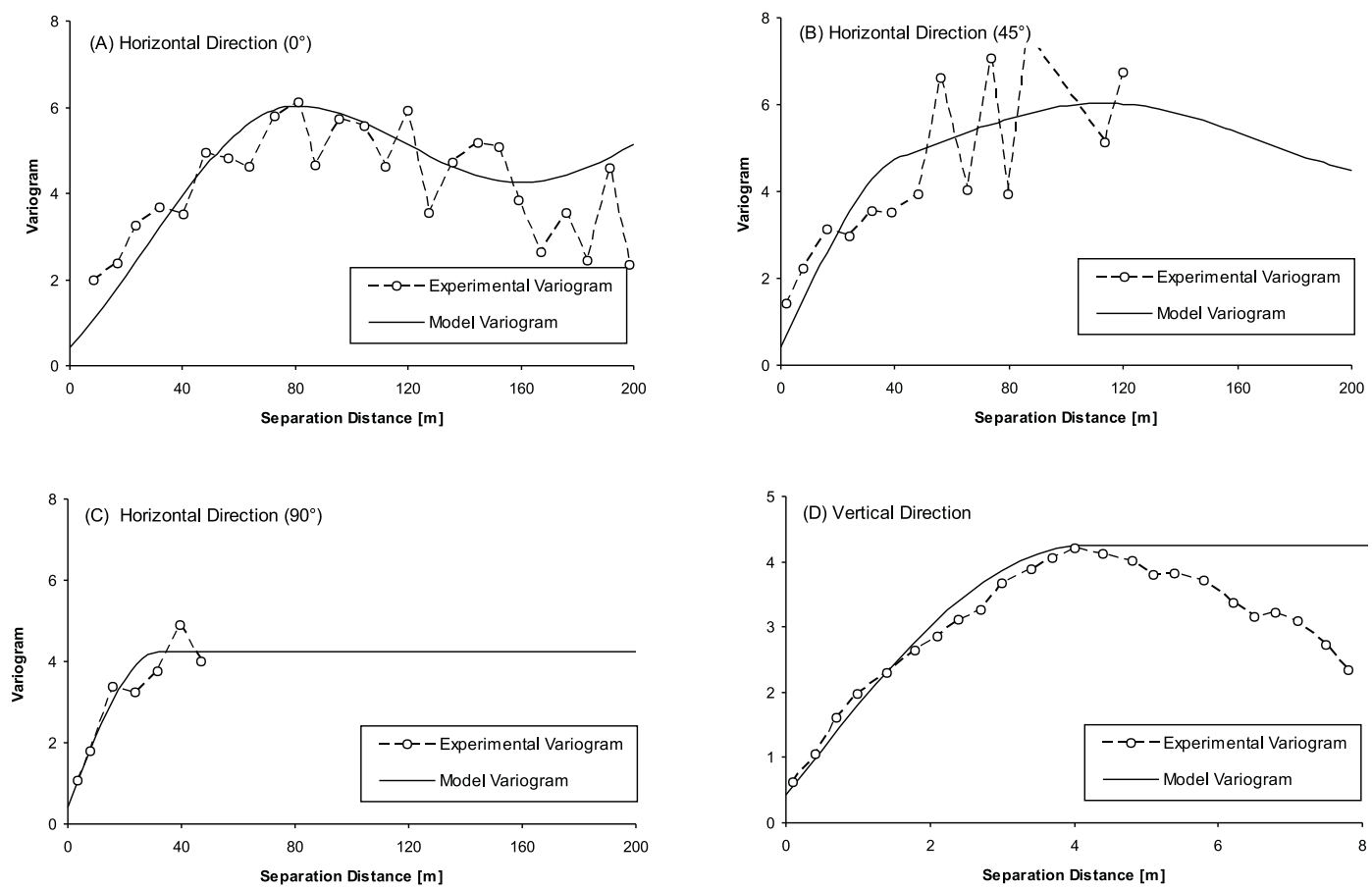

Figure 2: Horizontal and vertical experimental variograms, and fitted model, for the $\ln K$ flowmeter data. The rotation angle of the directional variograms is measured in degrees clockwise from the positive $y$-axis. 


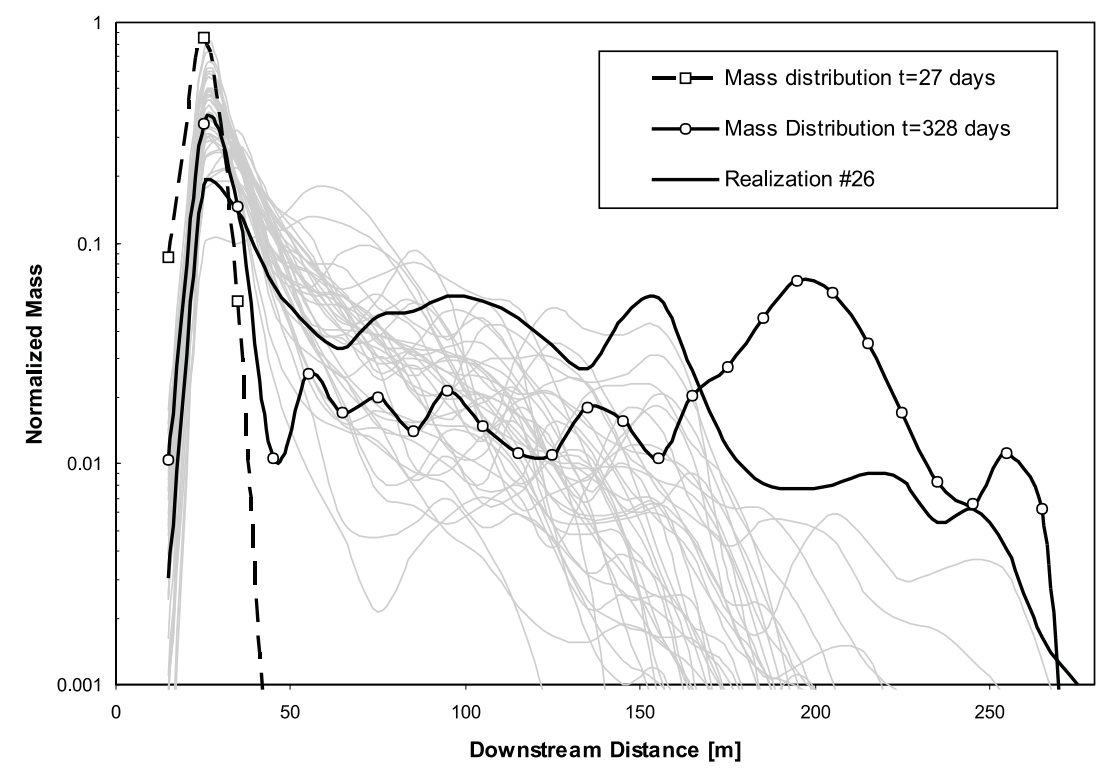

Figure 3: Longitudinal mass distribution profiles of the observed tritium plume at MADE, and predictions on several realizations of hydraulic conductivity. Each realization was generated (on natural-log space) over a grid of $110 \times 280 \times 70$ cells by sequential Gaussian simulation using the variogram model in Equation 1. 


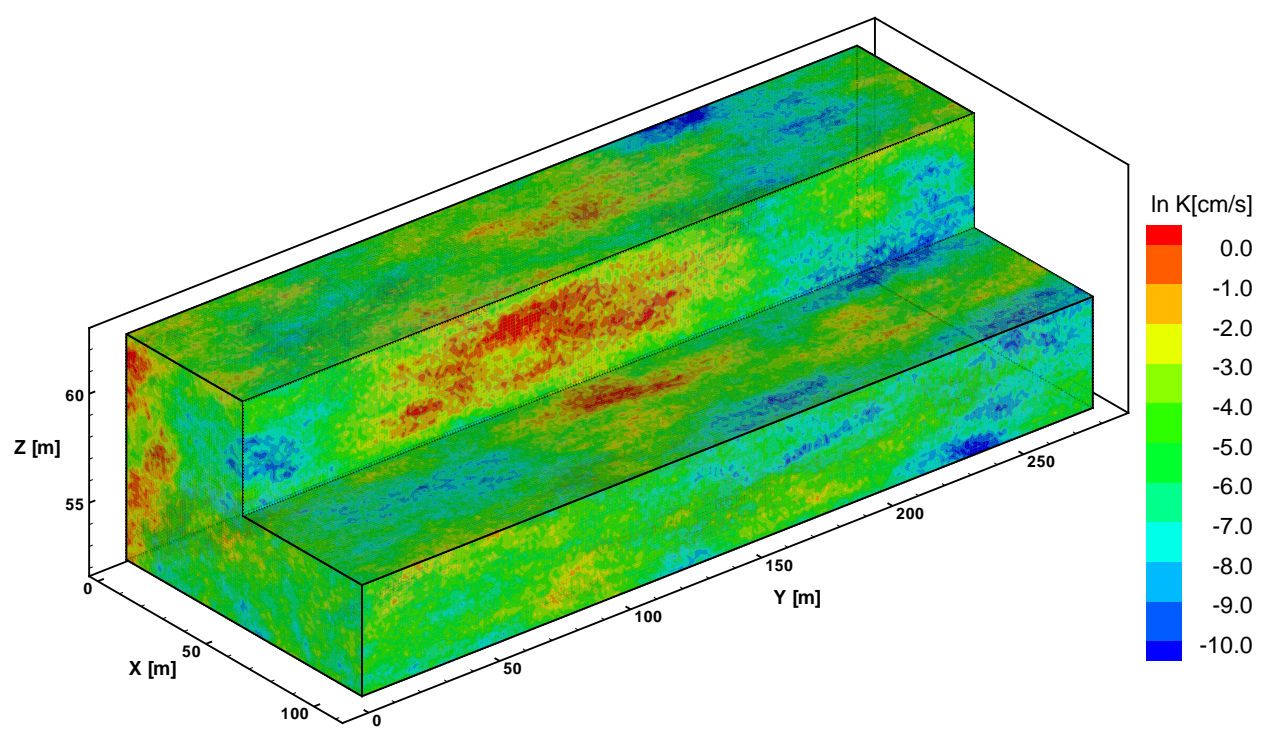

Figure 4: Realization \#26 of $\ln K$ from Salamon et al. (2007). This realization exhibits a strong solute tailing and it is used as the reference in the upscaling exercise. (The scale of the $z$-axis is exaggerated seven times for clarity.)

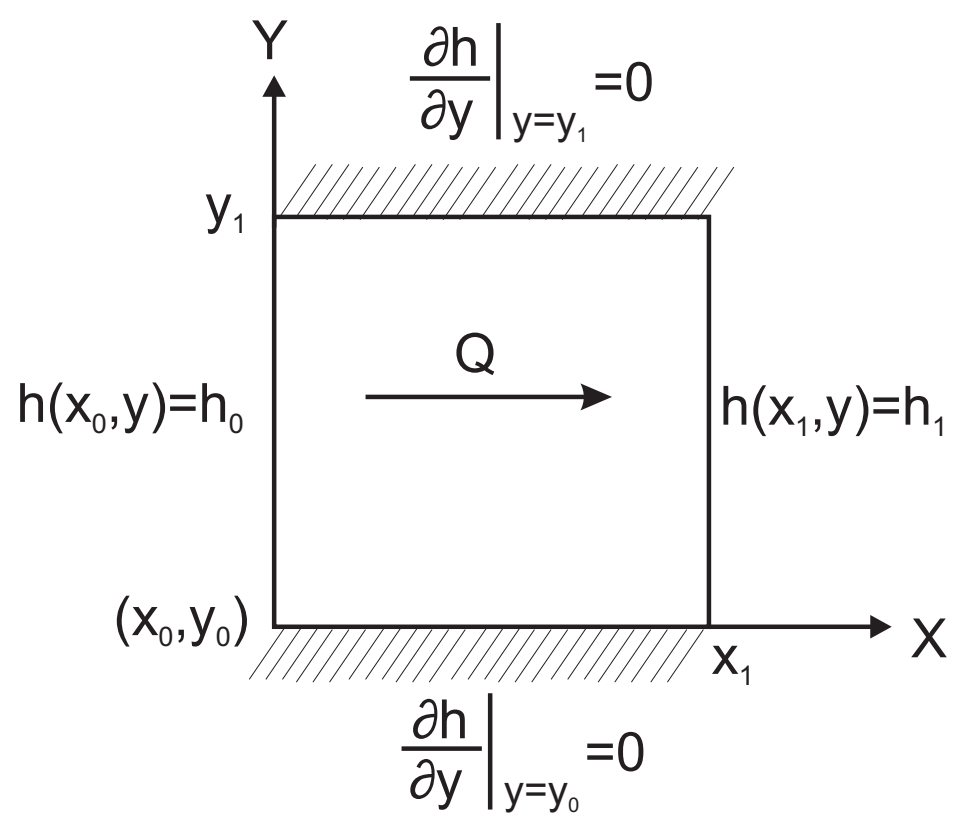

Figure 5: Boundary conditions that would be used in 2D for the local flow model when performing the simple-Laplacian upscaling in order to determine the $x$-component of the hydraulic conductivity tensor. In the simple-Laplacian approach, it is always assumed that the principal directions of the conductivity tensor are parallel to the reference axes. 

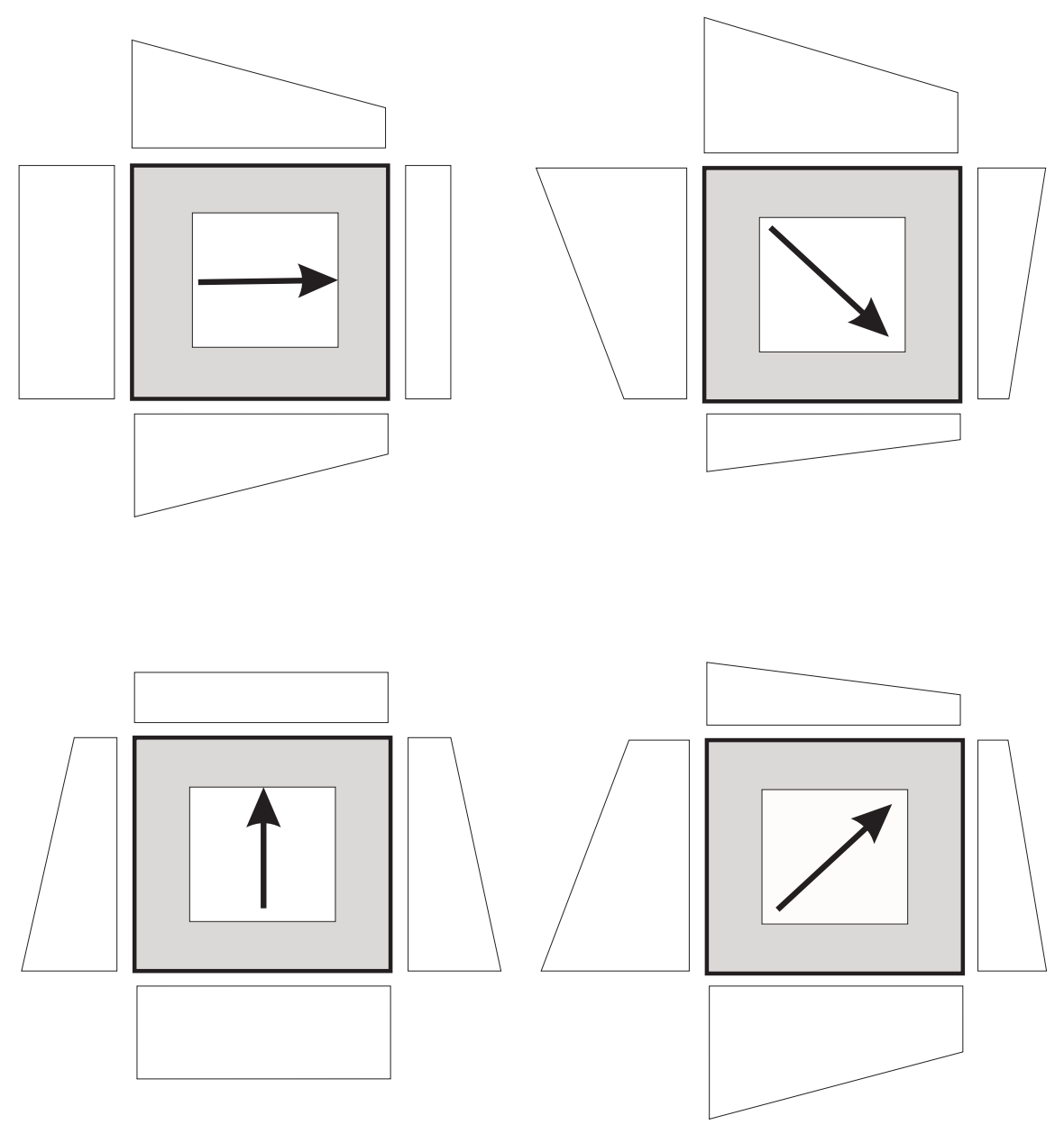

Figure 6: An example of four boundary condition sets that could be used in 2D for the local flow models when performing the Laplacian-with-skin upscaling. The white area is the block being upscaled, and the gray area is the skin region; the arrows indicate the (negative) mean head gradient induced by the prescribed head boundary conditions, and the shapes on the sides of the block indicate the magnitude of the prescribed heads given by tilting planes with gradients opposite to the arrows. 

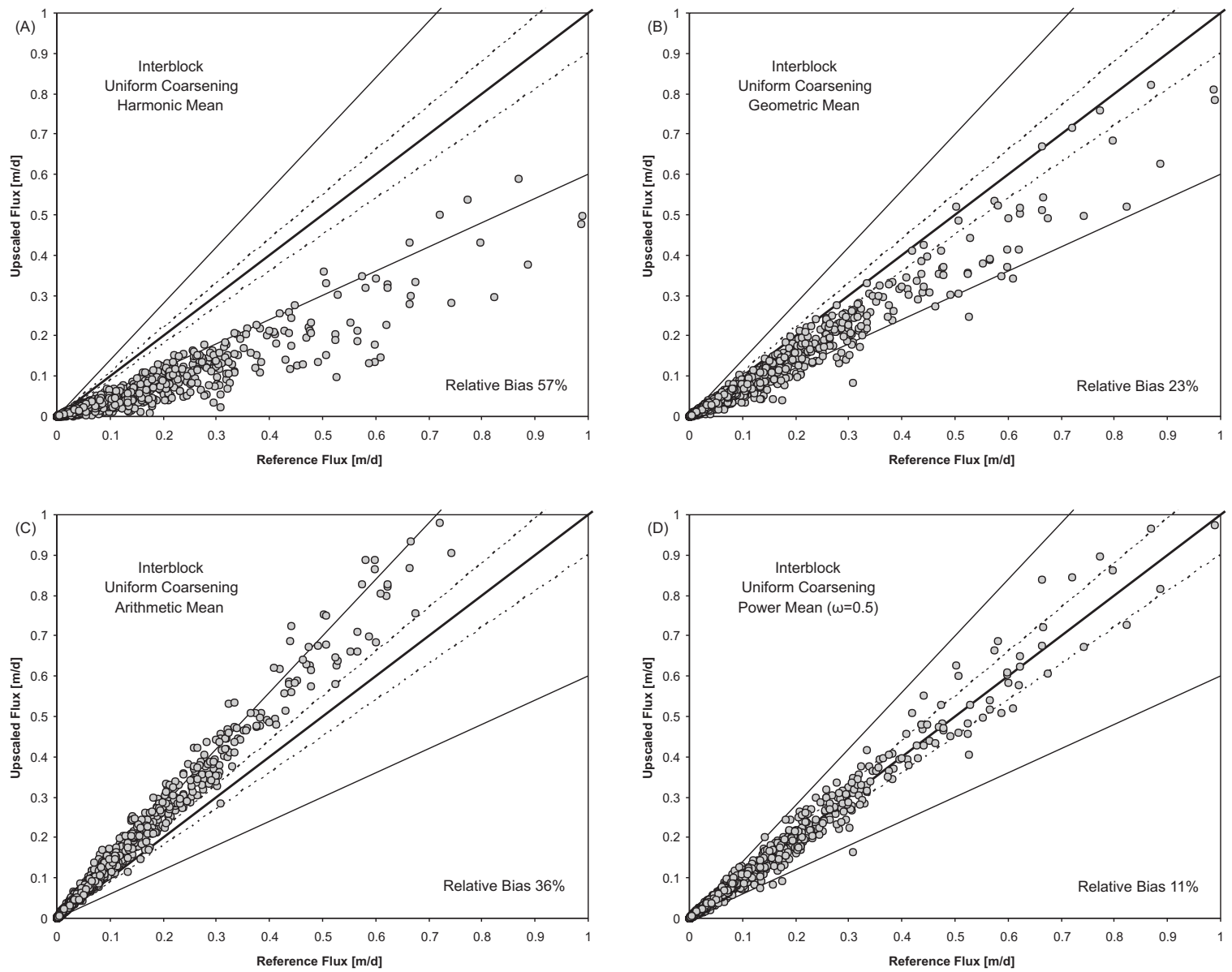

Figure 7: Flow comparison at the fine and coarse scales using simple averaging upscaling approaches. All circles within the dashed lines correspond to coarse scale values that deviate less than $10 \%$ from the reference ones; similarly, all circles within the outer solid lines correspond to coarse scale values that deviate less than $40 \%$. The average relative bias, as defined in Equation 10 , is reported in the lower right corner of each box. 


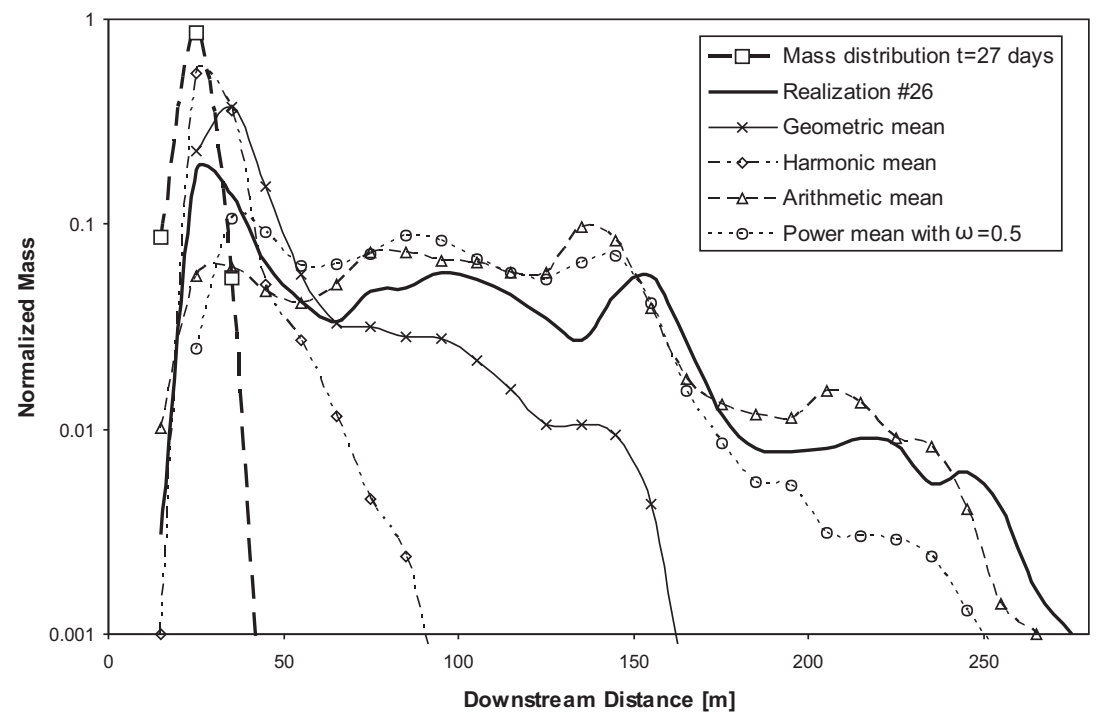

Figure 8: Longitudinal mass distribution profiles of the tritium plume from the fine scale reference realization, and predictions by some simple averaging upscaling approaches at the coarse scale for $t=328$ days. 

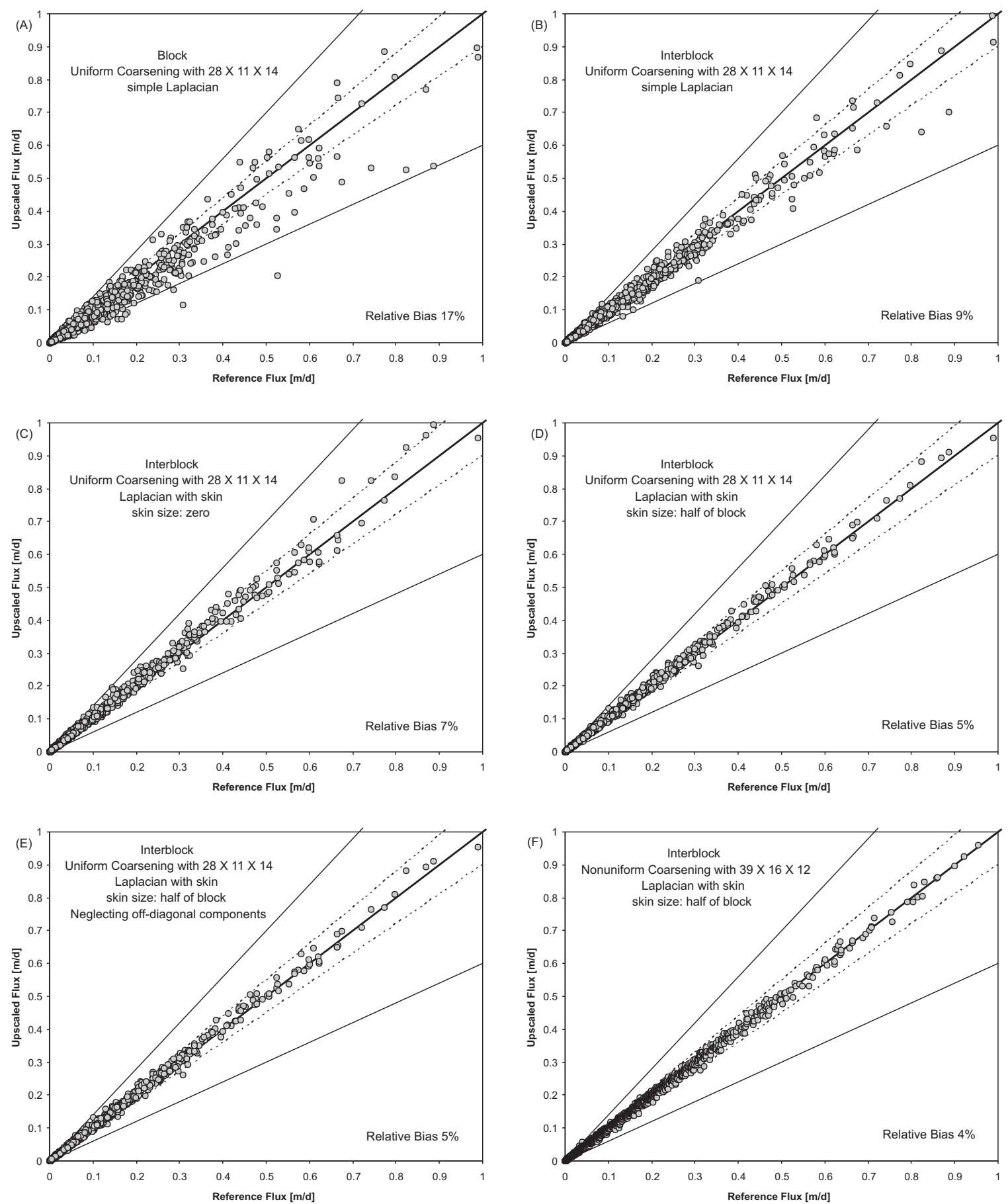

Figure 9: Flow comparison at the fine and coarse scales using Laplacian-based upscaling approaches. All circles within the dashed lines correspond to coarse scale values that deviate less than $10 \%$ from the reference ones; similarly, all circles within the outer solid lines correspond to coarse scale values that deviate less than $40 \%$. The average relative bias, as defined in Equation 10 , is reported in the lower right corner of each box. 


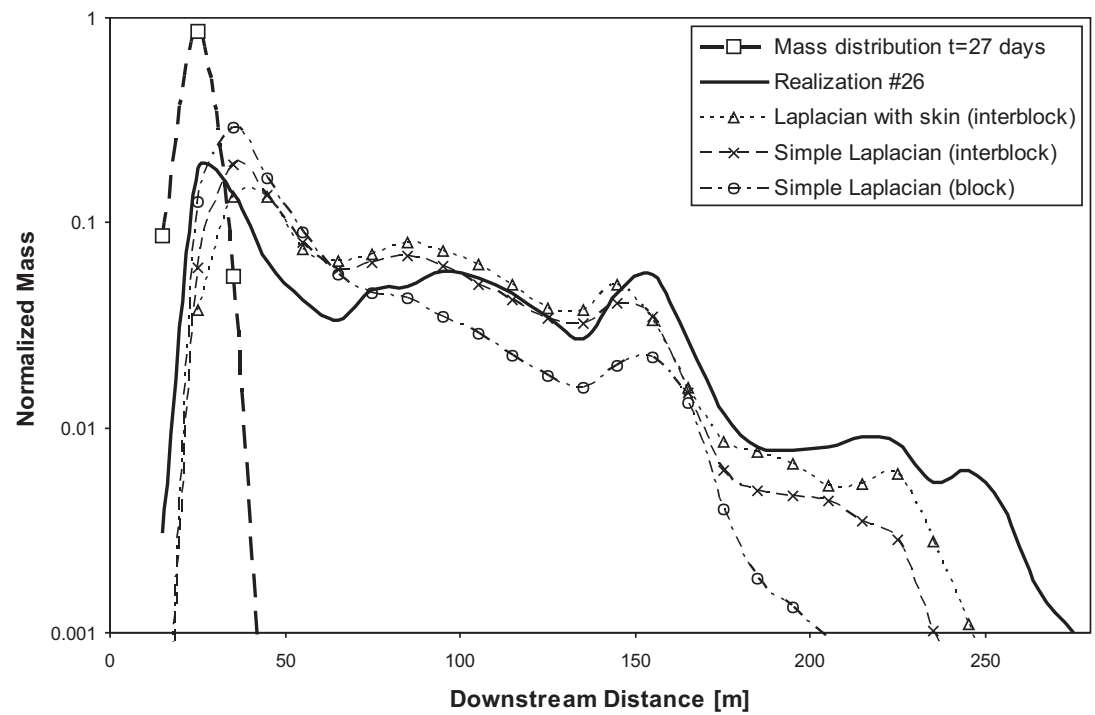

Figure 10: Longitudinal mass distribution profiles of the tritium plume from the fine scale reference realization, and predictions by some Laplacian-based upscaling approaches at the coarse scale, for $t=328$ days.

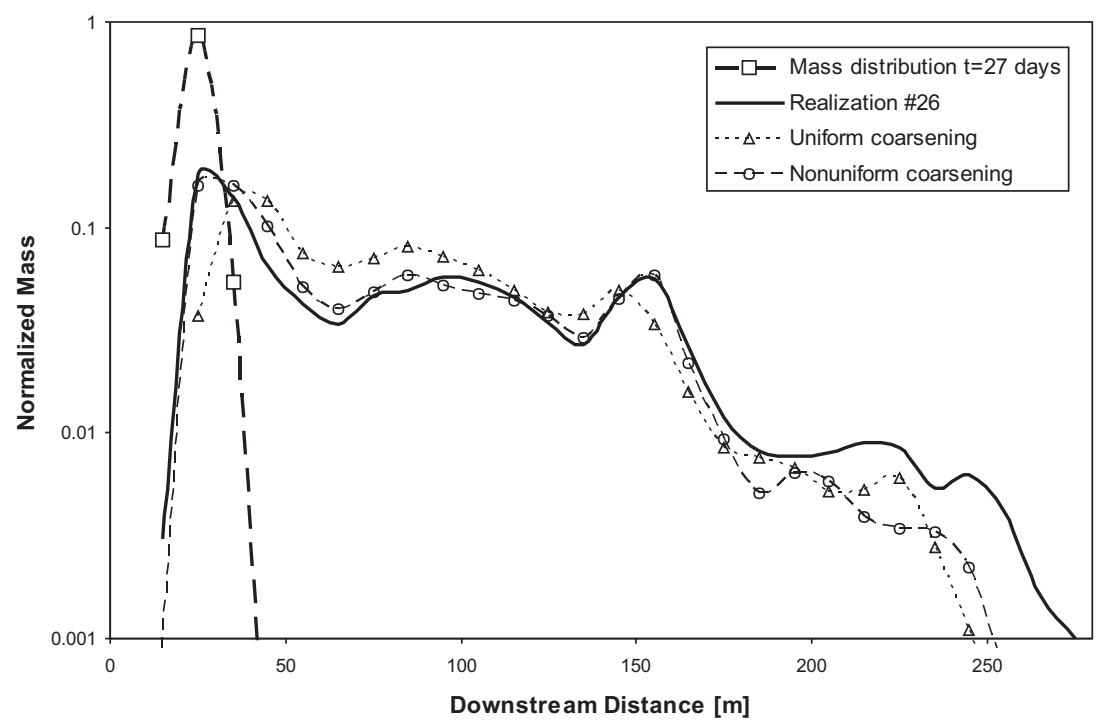

Figure 11: Longitudinal mass distribution profiles of the tritium plume from the fine scale reference realization, and predictions on uniform and non-uniform coarse scale grids, for $t=328$ days. 

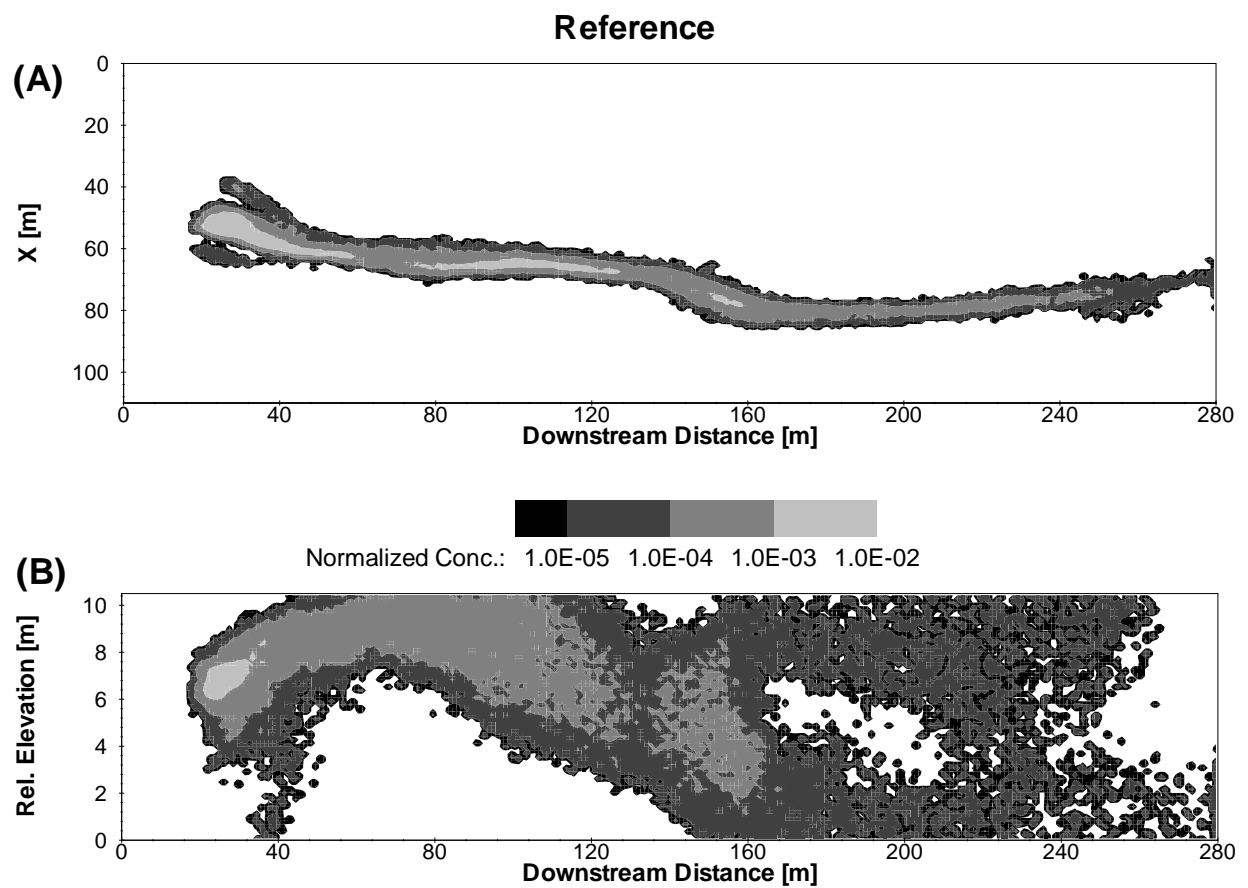

Figure 12: Transport in the fine scale reference realization for $t=328$ days. (A) Depth-integrated normalized concentration distribution. (B) Laterally-integrated normalized concentration distribution. 

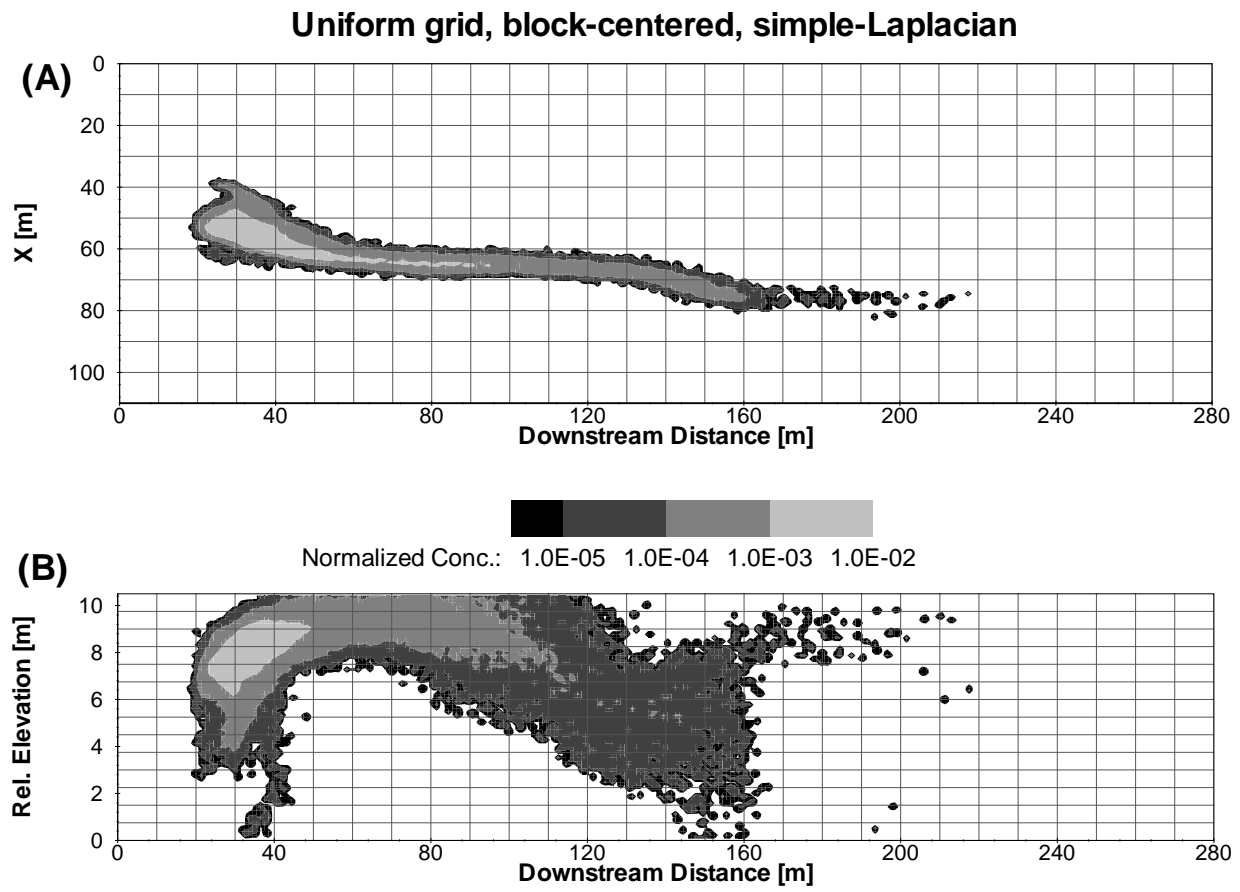

Figure 13: Transport at the coarse scale after upscaling the reference realization on a uniform grid using a block-centered simpleLaplacian approach for $t=328$ days. (A) Depth-integrated normalized concentration distribution. (B) Laterally-integrated normalized concentration distribution. 

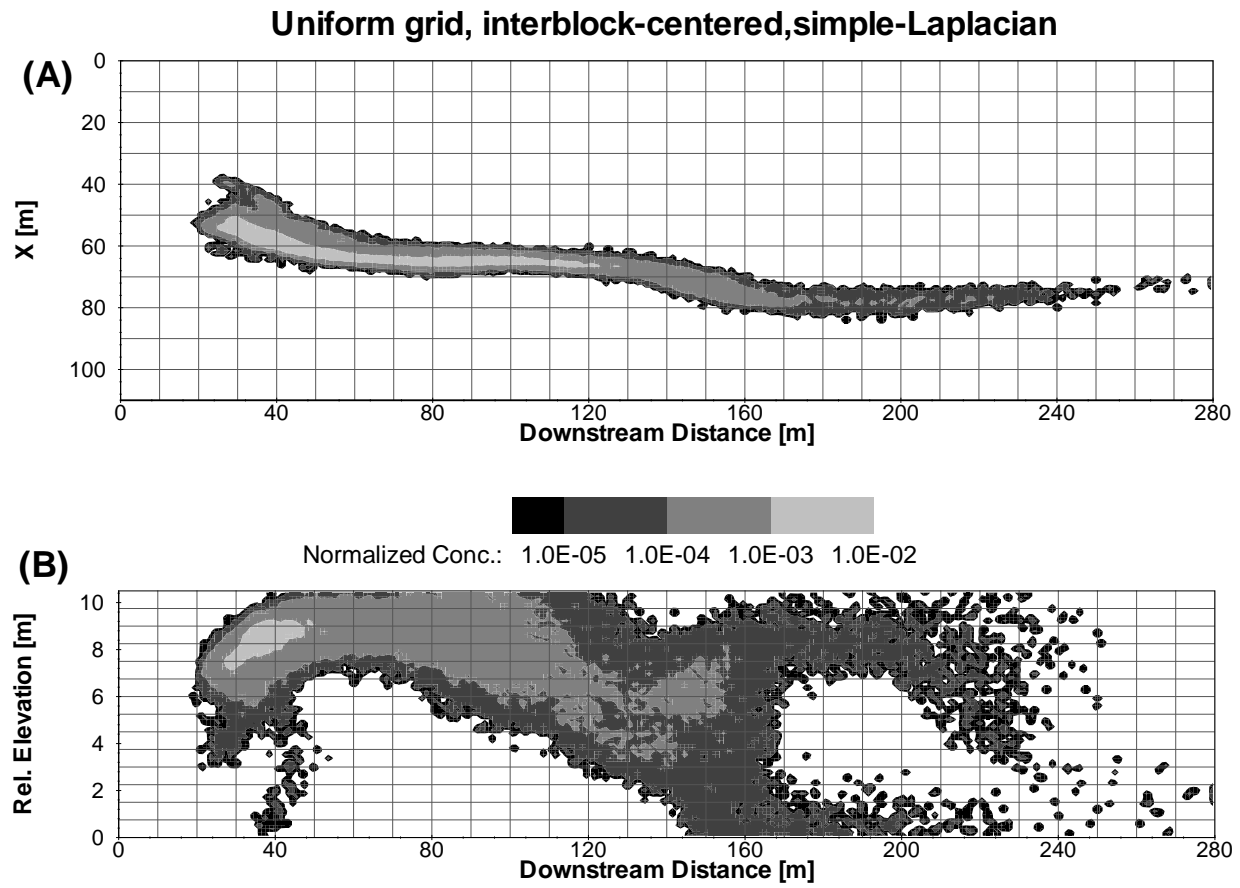

Figure 14: Transport at the coarse scale after upscaling the reference realization on a uniform grid using an interblock-centered simple-Laplacian approach for $t=328$ days. (A) Depth-integrated normalized concentration distribution. (B) Laterallyintegrated normalized concentration distribution. 

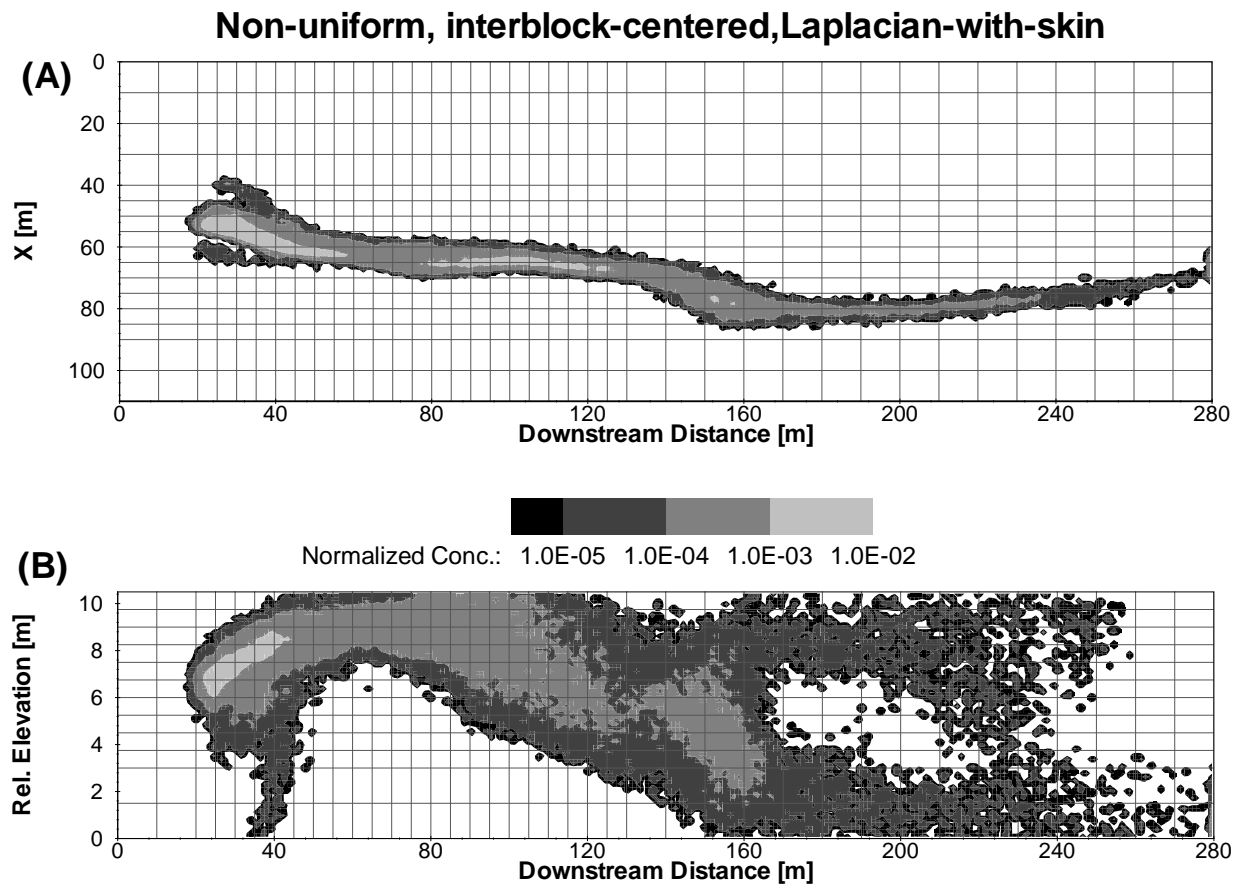

Figure 15: Transport at the coarse scale after upscaling the reference realization on a non-uniform grid using an interblockcentered Laplacian-with-skin approach for $t=328$ days.

(A) Depth-integrated normalized concentration distribution. (B) Laterally-integrated normalized concentration distribution. 


\section{Appendix A}

A nineteen-point block-centered finite-difference procedure for the solution of saturated groundwater steady flow in 3D with full tensor conductivities is described here. In the absence of sinks and sources, the partial differential equation governing flow in three-dimensions can be expressed as:

$\frac{\partial}{\partial x}\left(K_{x x} \frac{\partial h}{\partial x}+K_{x y} \frac{\partial h}{\partial y}+K_{x z} \frac{\partial h}{\partial z}\right)+\frac{\partial}{\partial y}\left(K_{x y} \frac{\partial h}{\partial x}+K_{y y} \frac{\partial h}{\partial y}+K_{y z} \frac{\partial h}{\partial z}\right)+\frac{\partial}{\partial z}\left(K_{x z} \frac{\partial h}{\partial x}+K_{y z} \frac{\partial h}{\partial y}+K_{z z} \frac{\partial h}{\partial z}\right)=0$

If this equation is discretized with a nineteen-point block-centered finite-difference stencil over a non-uniform grid of parallelpipedal blocks, the following equation results for a generic block $(i, j, k)$ of size $\left.\Delta x\right|_{i, j, k} \times$ $\left.\Delta y\right|_{i, j, k} \times\left.\Delta z\right|_{i, j, k}($ see Figure A-1):

$$
\begin{aligned}
& \frac{1}{\left.\Delta x\right|_{i, j, k}}\left[\left.\left(K_{x x} \frac{\partial h}{\partial x}+K_{x y} \frac{\partial h}{\partial y}+K_{x z} \frac{\partial h}{\partial z}\right)\right|_{i+1 / 2, j, k}-\left.\left(K_{x x} \frac{\partial h}{\partial x}+K_{x y} \frac{\partial h}{\partial y}+K_{x z} \frac{\partial h}{\partial z}\right)\right|_{i-1 / 2, j, k}\right]+ \\
& \frac{1}{\left.\Delta y\right|_{i, j, k}}\left[\left.\left(K_{x y} \frac{\partial h}{\partial x}+K_{y y} \frac{\partial h}{\partial y}+K_{y z} \frac{\partial h}{\partial z}\right)\right|_{i, j+1 / 2, k}-\left.\left(K_{x y} \frac{\partial h}{\partial x}+K_{y y} \frac{\partial h}{\partial y}+K_{y z} \frac{\partial h}{\partial z}\right)\right|_{i, j-1 / 2, k}\right]+ \\
& \frac{1}{\left.\Delta z\right|_{i, j, k}}\left[\left.\left(K_{x z} \frac{\partial h}{\partial x}+K_{y z} \frac{\partial h}{\partial y}+K_{z z} \frac{\partial h}{\partial z}\right)\right|_{i, j, k+1 / 2}-\left.\left(K_{x z} \frac{\partial h}{\partial x}+K_{y z} \frac{\partial h}{\partial y}+K_{z z} \frac{\partial h}{\partial z}\right)\right|_{i, j, k-1 / 2}\right]=0
\end{aligned}
$$

The hydraulic gradients at the interfaces are approximated by central differences from the heads at the nineteen blocks surrounding $(i, j, k)$, That is,

$$
\begin{aligned}
\left.\frac{\partial h}{\partial x}\right|_{i+1 / 2, j, k} & =\frac{h_{i, j+1, k}-h_{i, j-1, k}}{\left.\triangle x\right|_{i, j+1, k}+\left.2 \triangle x\right|_{i, j, k}+\left.\triangle x\right|_{i, j-1, k}}+\frac{h_{i+1, j+1, k}-h_{i+1, j-1, k}}{\left.\triangle x\right|_{i+1, j+1, k}+\left.2 \triangle x\right|_{i+1, j, k}+\left.\triangle x\right|_{i+1, j-1, k}} \\
\left.\frac{\partial h}{\partial y}\right|_{i+1 / 2, j, k} & =\frac{2\left(h_{i+1, j, k}-h_{i, j, k}\right)}{\left.\triangle y\right|_{i+1, j, k}+\left.\triangle y\right|_{i, j, k}} \\
\left.\frac{\partial h}{\partial z}\right|_{i+1 / 2, j, k} & =\frac{h_{i, j, k+1}-h_{i, j, k-1}}{\left.\triangle z\right|_{i, j, k+1}+\left.2 \triangle z\right|_{i, j, k}+\left.\triangle z\right|_{i, j, k-1}}+\frac{h_{i+1, j, k+1}-h_{i+1, j, k-1}}{\left.\triangle z\right|_{i+1, j, k+1}+\left.2 \triangle z\right|_{i+1, j, k}+\left.\triangle z\right|_{i+1, j, k-1}}
\end{aligned}
$$

The partial derivatives of the hydraulic head in the other five interfaces can be given by similar expressions. Substituting (A-3) into (A-2), multiplying both sides by $\left.\left.\left.\Delta x\right|_{i, j, k} \Delta y\right|_{i, j, k} \Delta z\right|_{i, j, k}$, and rearranging terms, the 


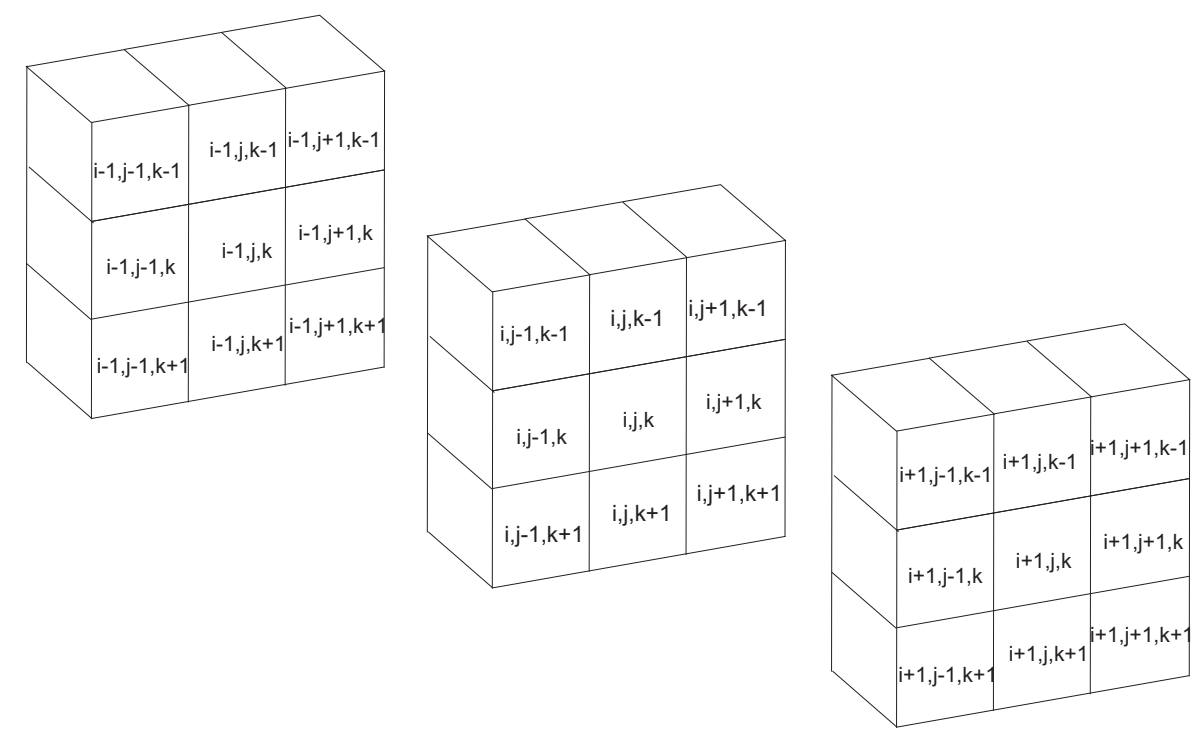

Figure A-1: Schematic illustration of the 3D finite-difference spatial discretization

nineteen-point results in:

$$
\begin{aligned}
& A h_{i, j+1, k}+B h_{i, j, k}+C h_{i+1, j+1, k}+D h_{i-1, j+1, k}+E h_{i+1, j, k}+F h_{i-1, j, k}+G h_{i, j+1, k+1}+ \\
& H h_{i, j+1, k-1}+I h_{i, j, k+1}+J h_{i, j, k-1}+K h_{i, j-1, k}+L h_{i+1, j-1, k}+M h_{i-1, j-1, k}+ \\
& N h_{i, j-1, k+1}+O h_{i, j-1, k-1}+P h_{i+1, j, k+1}+Q h_{i+1, j, k-1}+R h_{i-1, j, k+1}+S h_{i-1, j, k-1}=0
\end{aligned}
$$

where A, B, ..., S are function of the block sizes and interface hydraulic conductivity components. Equation (A-4) is written for all the nodes within the aquifer, except for those for which head is prescribed, resulting in a set of linear equations.

Acknowledgements The authors gratefully acknowledge the financial support by ENRESA (project 0079000029). The second author also acknowledges the financial support from China Scholarship Council. The two anonymous reviewers are gratefully acknowledged for their comments which helped improving the final version of the manuscript. 


\section{References}

Adams, E. E., Gelhar, L. W., 1992. Field study of dispersion in a heterogeneous aquifer 2. spatial moments analysis. Water Resources Research 28 (12), 3293-3307.

Anderman, E. R., Kipp, K. L., Hill, M. C., Valstar, J., Neupauer, R. M., 2002. MODFLOW-2000, the US geological survey modular Ground-Water Model Documentation of the Model-Layer Variable-Direction horizontal anisotropy (LVDA) capability of the Hydrogeologic-Unit flow (HUF) package. US Geological Survey, Open file Report, 02-409.

Appel, C. A., 1976. A note on computing finite difference interblock transmissivities. Water Resources Research $12(3), 561-563$.

Bachu, S., Cuthiell, D., 1990. Effects of Core-Scale heterogeneity on steady state and transient fluid flow in porous media: Numerical analysis. Water Resources Research 26 (5), 863-874.

Baeumer, B., Benson, D. A., Meerschaert, M. M., Wheatcraft, S. W., 2001. Subordinated advectiondispersion equation for contaminant transport. Water Resources Research 37 (6), 1543-1550.

Barlebo, H. C., Hill, M. C., Rosbjerg, D., 2004. Investigating the macrodispersion experiment (MADE) site in columbus, mississippi, using a three-dimensional inverse flow and transport model. Water Resources Research 40 (4), W04211.

Bear, J., 1972. Dynamics of fluids in porous media. American Elsevier Pub. Co., New York.

Begg, S. H., Carter, R. B. R. C., Dranfield, P. B. A., 1989. Assigning effective values to simulator gridblock parameters for heterogeneous reservoirs. SPE (Society of Petroleum Engineers) Reservoir Engineering.

Benson, D. A., Schumer, R., Meerschaert, M. M., Wheatcraft, S. W., 2001. Fractional dispersion, levy motion, and the MADE tracer tests. Transport in porous media 42 (1), 211-240.

Berkowitz, B., Scher, H., 1998. Theory of anomalous chemical transport in random fracture networks. Physical Review E 57 (5), 5858-5869.

Bierkens, M. F. P., Weerts, H. J. T., 1994. Block hydraulic conductivity of cross-bedded fluvial sediments. Water Resources Research 30 (10), 2665-2678. 
Boggs, J., Beard, L., Waldrop, W., Stauffer, T., MacIntyre, W., Antworth, C., 1993. Transport of tritium and four organic compounds during a natural gradient experiment (MADE-2), EPRI Report TR-101998. Electric Power Research Institute, Palo Alto, CA 94304.

Boggs, J. M., Adams, E. E., 1992. Field study of dispersion in a heterogeneous aquifer 4. investigation of adsorption and sampling bias. Water Resources Research 28 (12), 3325-3336.

Boggs, J. M., Young, S. C., Beard, L. M., Gelhar, L. W., Rehfeldt, K. R., Adams, E. E., 1992. Field study of dispersion in a heterogeneous aquifer 1. overview and site description. Water Resources Research 28 (12), $3281-3291$.

Bourgeat, A., 1984. Homogenized behavior of two-phase flows in naturally fractured reservoirs with uniform fractures distribution. Computer Methods in Applied Mechanics and Engineering 47 (1-2), 205-216.

Bouwer, H., 1969. Planning and interpreting soil permeability measurements. Journal of the Irrigation and Drainage Division, ASCE 95, 391-402.

Cardwell, W. T., Parsons, R. L., 1945. Averaging permeability of heterogeneous oil sands. Transactions of the American Institute of Mining, Metallurgical and Petroleum Engineers 160, 34-42.

Chen, Y., Durlofsky, L. J., Gerritsen, M., Wen, X. H., 2003. A coupled local-global upscaling approach for simulating flow in highly heterogeneous formations. Advances in Water Resources 26 (10), 1041-1060.

Desbarats, A. J., 1987. Numerical estimation of effective permeability in Sand-Shale formations. Water Resources Research 23 (2), 273-286.

Desbarats, A. J., 1988. Estimation of effective permeabilities in the lower stevens formation of the paloma field, san joaquin valley, california. SPE Reservoir Engineering 3 (4), 1301-1307.

Desbarats, A. J., 1992. Spatial averaging of hydraulic conductivity in three-dimensional heterogeneous porous media. Mathematical Geology 24 (3), 249-267.

Deutsch, C. V., 1989. Calculating effective absolute permeability in Sand-Shale sequences. SPE Formation Evaluation 4 (3), 343-348.

Durlofsky, L. J., 1991. Numerical calculations of equivalent grid block permeability tensors for heterogeneous porous media. Water Resources Research 27 (5), 699-708. 
Durlofsky, L. J., Jones, R. C., Milliken, W. J., 1997. A nonuniform coarsening approach for the scale-up of displacement processes in heerogeneous porous media. Advances in Water Resources 20 (5-6), 335-347.

Eggleston, J., Rojstaczer, S., 1998. Identification of Large-Scale hydraulic conductivity trends and the influence of trends on contaminant transport. Water Resources Research 34 (9), 2155-2168.

Feehley, C. E., Zheng, C., Molz, F. J., 2000. A dual-domain mass transfer approach for modeling solute transport in heterogeneous aquifers: Application to the macrodispersion experiment (MADE) site. Water Resources Research 36 (9), 2501-2515.

Fernàndez-Garcia, D., Gómez-Hernández, J. J., 2007. Impact of upscaling on solute transport: Traveltimes, scale dependence of dispersivity, and propagation of uncertainty. Water Resources Research 43 (2).

Fernàndez-Garcia, D., Illangasekare, T. H., Rajaram, H., 2005. Differences in the scale dependence of dispersivity and retardation factors estimated from forced-gradient and uniform flow tracer tests in threedimensional physically and chemically heterogeneous porous media. Water Resources Research 41 (3), W03012.

Fernàndez-Garcia, D., Llerar-Meza, G., Gómez-Hernández, J. J., 2009. Upscaling transport with mass transfer models: Mean behavior and propagation of uncertainty. Water Resources Research 45, W10411.

Flodin, E. A., Durlofsky, L. J., Aydin, A., 2004. Upscaled models of flow and transport in faulted sandstone: boundary condition effects and explicit fracture modelling. Petroleum Geoscience 10 (2), 173-181.

Freeze, R. A., Cherry, J. A., 1979. Groundwater. Prentice-Hall.

Garcia, M., Journel, A. G., Aziz, K., 1992. Automatic grid generation for modeling reservoir heterogeneities. SPE Reservoir Engineering 1992, 278-284.

Gillham, R. W., Robin, M. J. L., Dytynyshyn, D. J., Johnston, H. M., 1984. Diffusion of nonreactive and reactive solutes through fine-grained barrier materials. Canadian Geotechnical Journal 21 (3), 541-550.

Gómez-Hernández, J. J., 1991. A stochastic approach to the simulation of block conductivity values conditioned upon data measured at a smaller scale. Ph.D. thesis, Stanford University.

Gómez-Hernández, J. J., Journel, A. G., 1993. Joint sequential simulation of multi-Gaussian fields. Geostatistics Troia 92 (1), 85-94. 
Gómez-Hernández, J. J., Wen, X. H., 1994. Probabilistic assessment of travel times in groundwater modeling. J. of Stochastic Hydrology and Hydraulics 8 (1), 19-56.

Guan, J., Molz, F. J., Zhou, Q., Liu, H. H., Zheng, C., 2008. Behavior of the mass transfer coefficient during the MADE-2 experiment: New insights. Water Resources Research 44, W02423.

Harbaugh, A. W., Banta, E. R., Hill, M. C., McDonald, M. G., 2000. MODFLOW-2000, the U.S. Geological Survey modular ground-water model. English. U.S. Geological Survey, Branch of Information Services, Reston, VA, Denver, CO.

Harvey, C., Gorelick, S. M., 2000. Rate-Limited mass transfer or macrodispersion: Which dominates plume evolution at the macrodispersion experiment (MADE) site? Water Resources Research 36 (3), 637-650.

Hill, M. C., 1990. Preconditioned conjugate gradient 2 (PCG2). A computer program for solving groundwater flow equations: US Geological Survey Water-Resources Investigations Report, 98-4048.

Hill, M. C., Barlebo, H. C., Rosbjerg, D., 2006. Reply to comment by F. Molz et al. on investigating the macrodispersion experiment (MADE) site in columbus, mississippi, using a three-dimensional inverse flow and transport model. Water Resources Research 42 (6), W06604.

Indelman, P., Abramovich, B., 1994. Nonlocal properties of nonuniform averaged flows in heterogeneous media. Water Resour. Res. 30 (12), 3385-3393.

URL http://dx.doi.org/10.1029/94WR01782

Jourde, H., Flodin, E. A., Aydin, A., Durlofsky, L. J., Wen, X. H., 2002. Computing permeability of fault zones in eolian sandstone from outcrop measurements. AAPG bulletin 86 (7), 1187-1200.

Journel, A. G., Deutsch, C. V., Desbarats, A. J., 1986. Power averaging for block effective permeability. SPE 15128.

LaBolle, E. M., Fogg, G. E., Tompson, A. F., 1996. Random-walk simulation of transport in heterogeneous porous media: Local mass-conservation problem and implementation methods. Water Resources Research $32(3), 583-593$.

Lake, L. W., 1988. The origins of anisotropy. J. of Petr. Techn. April, 395-396.

Li, L., Zhou, H., Gómez-Hernández, J. J., 2010. Steady-state groundwater flow modeling with full tensor conductivities using finite differences. Computers \& Geosciences 36 (10), 1211-1223. 
Li, L., Zhou, H., Gómez-Hernández, J. J., 2011. Transport upscaling using multi-rate mass transfer in three-dimensional highly heterogeneous porous media. Advances in Water Resources, in press, doi:10.1016/j.advwatres.2011.01.001.

Liu, G., Chen, Y., Zhang, D., 2008. Investigation of flow and transport processes at the MADE site using ensemble kalman filter. Advances in Water Resources 31 (7), 975-986.

Llopis-Albert, C., Capilla, J. E., 2009. Gradual conditioning of non-Gaussian transmissivity fields to flow and mass transport data: 3. application to the macrodispersion experiment (MADE-2) site, on Columbus Air Force Base in Mississippi (USA). Journal of Hydrology 371 (1-4), 75-84.

Matheron, G., 1967. Elements pour une theorie des milieux poreux. Masson et Cie.

Molz, F. J., Zheng, C., Gorelick, S. M., Harvey, C. F., 2006. Comment on Investigating the macrodispersion experiment (MADE) site in columbus, mississippi, using a three-dimensional inverse flow and transport model by Heidi Christiansen Barlebo, Mary C. Hill, and Dan Rosbjerg. Water Resources Research 42 (6), W06603.

Neuman, S. P., Orr, S., 1993. Prediction of steady state flow in nonuniform geologic media by conditional moments: Exact nonlocal formalism, effective conductivities, and weak approximation. Water Resour. Res. 29 (2), 341-364.

Press, W. H., Flannery, B. P., Teukolsky, S. A., Vetterling, W. T., 1988. Numerical recipes in C. Cambridge University Press, Cambridge.

Rehfeldt, K. R., Boggs, J. M., Gelhar, L. W., 1992. Field study of dispersion in a heterogeneous aquifer 3. geostatistical analysis of hydraulic conductivity. Water Resources Research 28 (12), 3309-3324.

Renard, P., Marsily, G. D., 1997. Calculating equivalent permeability: A review. Advances in Water Resources $20(5-6), 253-278$.

Romeu, R. K., Noetinger, B., 1995. Calculation of internodal transmissivities in finite difference models of flow in heterogeneous porous media. Water Resources Research 31 (4), 943-959.

Salamon, P., Fernàndez-Garcia, D., Gómez-Hernández, J. J., 2006. A review and numerical assessment of the random walk particle tracking method. Journal of Contaminant Hydrology 87 (3-4), 277-305. 
Salamon, P., Fernàndez-Garcia, D., Gómez-Hernández, J. J., 2007. Modeling tracer transport at the MADE site: the importance of heterogeneity. Water Resources Research 30 (8).

Sánchez-Vila, X., Carrera, J., Girardi, J. P., 1996. Scale effects in transmissivity. Journal of Hydrology $183(1-2), 1-22$.

Sánchez-Vila, X., Guadagnini, A., Carrera, J., 2006. Representative hydraulic conductivities in saturated groundwater flow. Reviews of Geophysics 44 (3).

Schumer, R., Benson, D. A., Meerschaert, M. M., Baeumer, B., 2003. Fractal mobile/immobile solute transport. Water Resources Research 39 (10), 1296.

Vermeulen, P. T. M., Stroet, C. B. M. T., Heemink, A. W., 2006. Limitations to upscaling of groundwater flow models dominated by surface water interaction. Water Resources Research 42 (10), W10406.

Warren, J. E., Price, H. S., 1961. Flow in heterogeneous porous media. Society of Petroleum Engineering Journal 1, 153-169.

Wen, X. H., Durlofsky, L. J., Edwards, M., 2003. Use of border regions for improved permeability upscaling. Mathematical Geology 35 (5), 521-547.

Wen, X. H., Gómez-Hernández, J. J., 1996a. The constant displacement scheme for tracking particles in heterogeneous aquifers. Groundwater 34 (1), 135-142.

Wen, X. H., Gómez-Hernández, J. J., 1996b. Upscaling hydraulic conductivities: An overview. Journal of Hydrology 183 (1-2), ix-xxxii.

Wen, X. H., Gómez-Hernández, J. J., 1998. Upscaling conductivities in cross-bedded formations. Mathematical Geology 30 (2), 181-212.

Zhou, H., Li, L., Gómez-Hernández, J. J., 2010. Three-dimensional hydraulic conductivity upscaling in groundwater modelling. Computers \& Geosciences 36 (10), 1224-1235. 